\title{
MESA NTMS $1^{\circ} \times 2^{\circ}$ QUADRANGLE AREA
}

\section{SUPPLEMENTAL DATA REPORT}

\author{
NATIONAL URANIUM RESOURCE \\ EVALUATION PROGRAM
}

\section{HYDROGEOCHEMICAL AND STREAM SEDIMENT RECONNAISSANCE}

G. R. KOLLER

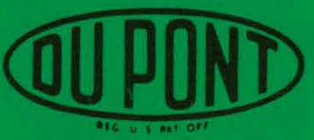

E. I. du Pont de Nemours \& Co. (Inc.) Savannah River Laboratory Aiken, South Carolina 29801 


\section{DISCLAIMER}

This report was prepared as an account of work sponsored by an agency of the United States Government. Neither the United States Government nor any agency Thereof, nor any of their employees, makes any warranty, express or implied, or assumes any legal liability or responsibility for the accuracy, completeness, or usefulness of any information, apparatus, product, or process disclosed, or represents that its use would not infringe privately owned rights. Reference herein to any specific commercial product, process, or service by trade name, trademark, manufacturer, or otherwise does not necessarily constitute or imply its endorsement, recommendation, or favoring by the United States Government or any agency thereof. The views and opinions of authors expressed herein do not necessarily state or reflect those of the United States Government or any agency thereof. 


\section{DISCLAIMER}

Portions of this document may be illegible in electronic image products. Images are produced from the best available original document. 


\section{FOREWORD}

This supplemental data report is released without standard editorial and technical review in order to make the information available as soon as possible to interested organizations and to assist the search for uranium resources.

\section{DISCLAIMER}

This report was prepared as an account of work sponsorad by the Unitec States Government. Neither the United States nor the United States De. partment of Energy, nor any of their employees, make any warranty, ex. press or implied, or assumes any legal liability or responsibility for the accuracy, completeness, or usefulness of any information, apparatus, pro. duct, or process disclosed, or represents that its use would not infringe privately owned rights. Reference herein to any specific commercial pro duct, process, or service by trade name, mark, manufacturer, or otherwise, does not necessarily constitute or imply its endorsement, recommendation. or favoring by the United States Government or any agency thereof. The views and opinions of authors expressed herein do not necessarily state or reflect those of the United States Government or any agency thereof.

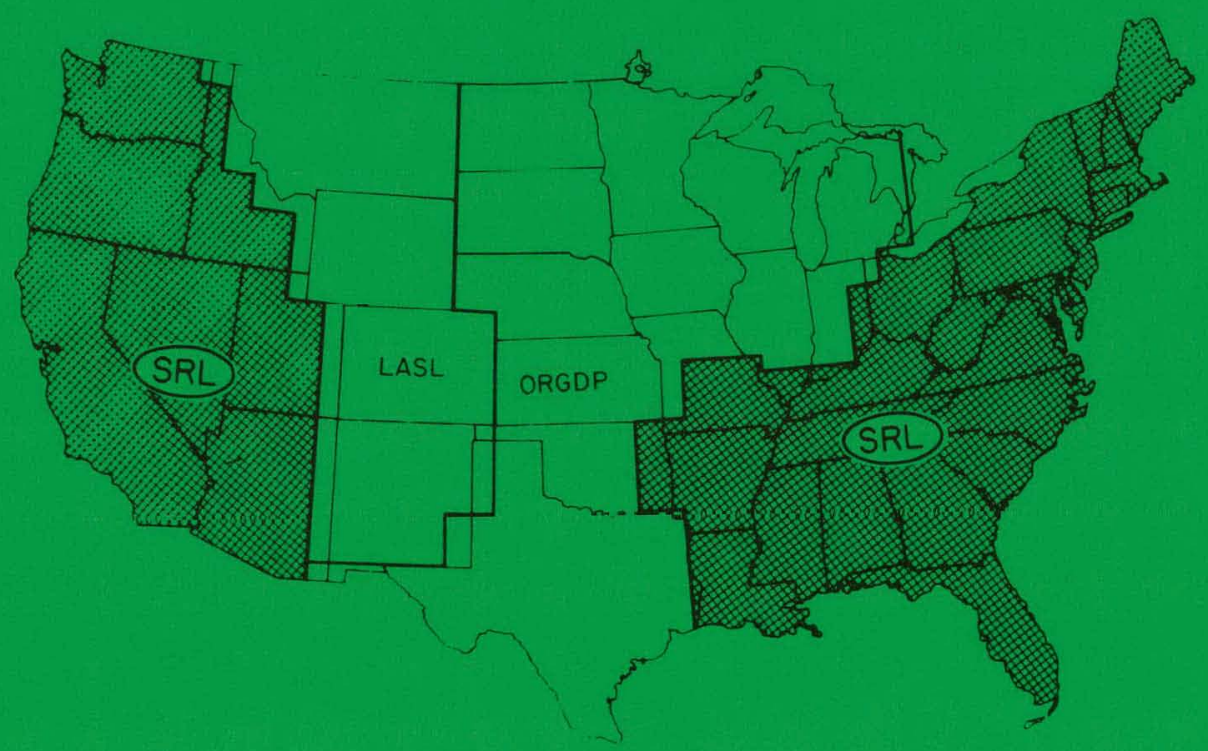




\section{SUPPLEMENTAL DATA REPORT}

\section{NATIONAL URANIUM RESOURCE EVALUATION PROGRAM}

HYDROGEOCHEMICAL AND STREAM SEDIMENT RECONNAISSANCE

by

G. R. Koller

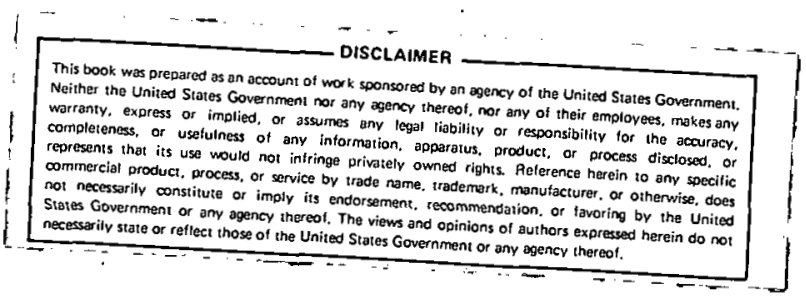

Approved by:

M. L. Hyder, Manager

Analytical Chemistry Division

Publication Date: January 1980

E. I. du Pont de Nemours \& Co. (Inc.)

Savannah River Laboratory Aiken, South Carolina 29801

PREPARED FOR THE U. S. DEPARTMENT OF ENERGY UNDER CONTRACT DE-AC09-76SR00001 
This data report presents supplemental analytical results for stream sediments and ground water. The samples were: collected as. part of the SRL-NURE reconnaissance in the National Topographic Map Series (NTMS) Mesa $1^{\circ} \times 2^{\circ}$ - quadrangle. Results are reported for 24 elements ( $\mathrm{Ag}, \mathrm{As}, \mathrm{Ba}, \mathrm{Be}, \mathrm{Ca}, \mathrm{Co}, \mathrm{Cr}, \mathrm{Cu}, \mathrm{K}, \mathrm{Li}, \mathrm{Mg}, \mathrm{Mo}$, $\mathrm{Nb}, \mathrm{Ni}, \mathrm{P}, \mathrm{Pb}, \mathrm{Se}, \mathrm{Sn}, \mathrm{Sr}, \mathrm{Th}, \mathrm{W}, \mathrm{Y}, \mathrm{Zn}$, and extractable U) in sediments and $31^{\prime}$ elements ( $\mathrm{Ag}, \mathrm{Al}, \mathrm{As}, \mathrm{B}, \mathrm{Ba}, \mathrm{Be}, \mathrm{Ca}, \mathrm{Ce}, \mathrm{Co}, \mathrm{Cr}$, $\mathrm{Cu}, \mathrm{Fe}, \cdot \mathrm{K}, \mathrm{Li}$; Mg, Mn, Mo, $\mathrm{Na}, \mathrm{Nb}^{2}, \mathrm{Ni}, \mathrm{P}, \mathrm{Sc}, \mathrm{Se}, \mathrm{Si}, \mathrm{Sr}$, Th, Ti, $\mathrm{V}, \mathrm{Y}, \mathrm{Zn}^{\prime}$ ' and $\mathrm{Z} \mathrm{r}$ ) as well as $\mathrm{pH}$, alkalinity, and conductivity in ground water. Field data and NAA data will be open-filed when they are available.

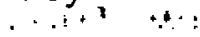

Microtiche cards have been placed in a pocket on the last page of this report. These cards contain,the following information:

- Cards marked Pg. 1, Pg. 2, and Pg. 3. contain histograms, cumulative frequency plots, and areal distribution plots for 'sediment samples.

- The card marked Plate 1 is a site-code map for sediment samples.

ERRATA:

Pages 36 and 37, Sample MMCDO31: UX $=6.4$ Th $=-100$

Pages. 40 and 41, Sample MMDA015: UX $=0.3$ Th $=70$

Pages 42 and 43, Sample MMDC015: UX $=0.5$ Th $=-70$

Pages 42 and 43, Sample MMDC031: IIX = 0.1 Th $=-55$.

Groundwater samples with type codes of 7 (see pp. 15 and 17) should have type codes of 52 .

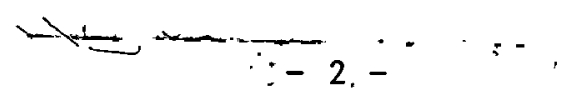


Introduction 5

Melliuds of Analyois 10

Description of Data Tables 10

SRL Identification Number 10

DOE Identification Number 15

Supplemental Elemental Analyses 15

References $\quad 18$

Appendix: Tabulation of Supplementary Analytical Data 19

I. Sediment Analyses from the Mesa Quadrangle

II. Ground Water Analyses from the Mesa.Quadrangle

\section{LIST OF FIGURES}

1 Location Map for the Mesa $1^{\circ} \times 2^{\circ}$ NTMS Quadrangle 7

2 Analysis Scheme for Supplementary Analyses 11

\section{LIST OF TABLES}

1 SRL Site Code Abbreviations for Western $1^{\circ} \times 2^{\circ}$ NTMS Quadrangles 8

2 Results of Certification Test for Supplementary Analyses 14

3 State Codes Used in DOE Identification Number 16

4 Type Codes Used in DOE Sample Identification Number 17 


\section{THIS PAGE}

\section{WAS INTENTIONALLY \\ LEFT BLANK}


SUPPLEMENTAL DATA REPORT, MESA $1^{\circ} \times 2^{\circ}$ NTMS QUADRANGLE

\section{INTRODOCTION}

The National Uranium Resource Evaluation (NURE) program was established to evaluate domestic uranium resources in the cont1nental United States and to Identify areas favorable for uranium exploration. The Grand Junction Office (GJO) of the Department of Energy (DOE) is responsible for administering and coordinating NURE program efforts. The Savannah RIver Laboratory (SRL) has responsibility for hydrogeochemical and stream sediment reconnalssance (HSSR) of 3.9 million square kilometers $(1,500,000$ square miles) in 37 eastern and western states. Other DOE laboratories are responsible for similar reconnalssance in the rest of the continental United States including Alaska. The significance of the distribution of uranium in natural waters and stream sediments will be assessed as an indicator of areas favorable for the location of uranium deposits.

The principal objectives of the NURE program are:

- Increase geologlc knowledge of U.S. uranium resources in reglons where uranium ore bodies are known to exist and are cand1date supplies under present and near-term market conditions.

- Complete assessment of lower cost potential uranium resources in the conterminous U.S. and Alaska.

- Improve rellabllity and validate resource estimates and increase confidence levels.

- Expand scope of uranium assessment to include higher cost and relatively unknown domestic resources that may be feasible uranium supply alternatives.

- Apply advanced technologies for detection and assessment of urani un resonirces.

DOE-GJO is responsible for administering and coordinating efforts to meet these objectives including distribution of reports. Inputs to the NURE program come from DOE prime contractors, DOEsponsored research and development, the uranium industry, U.S. Geological Survey, U.S. Bureau of Mines, other federal and state government agencies, and independent sources. 
The NURE program consists of six parts:

1. Hydrogeochemical and Stream Sediment Reconnaissance Survey

2. Aerial Radiometric Survey

3. Intermediate Grade Resource Studies

4. World Class Geologic Studies

5. Subsurface Geologic Investigation

6. Technology Application

The data presented here are reconnaissance data intended for use in identifying broad areas for further study. While care has been taken to provide reliable sampling and analyses, verification of individual analyses is beyond the scope of this report. The data should be viewed statistically because "one-point anomalies" may be misleading. Regional trends, however, should be reliable. With careful consideration of regional geology, these data should provide reliable guides to areas warranting further study.

This report is one of a serles presenting SRL reconnaissance supplementary data. Detalled descriptions of sampling techniques, fleld data, and neutron activation results (including $U, T h$, and Hf) will be reported in a subsequent basic data report for the Mesa quadrangle. Identification numbers (both SRL and DOE) and supplemental analysis data are presented in this report in tabular form. Page-size areal distribution plots of selected elements may be Included (as Part III of the Append1x) if deemed appropriate. All data will be avallable on magnetic tape from:

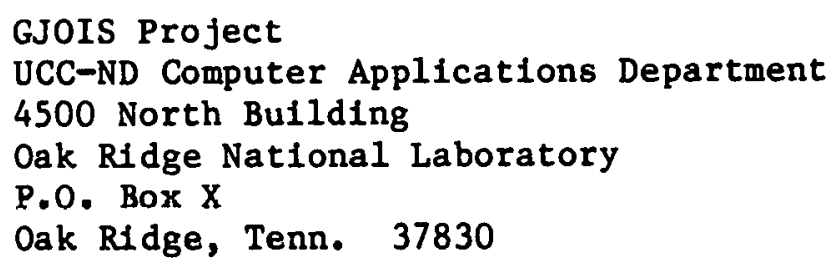

A summary of the SRL development program in support of the reconnaissance is avallable in $\bar{S} \bar{R} L-N \bar{U} \bar{R}$ progress reports (SRL-138).

The location of the Mesa quadrangle is shown in Figure 1.

Table 1 summarizes the quadrangle abbrevlations used in SRL sample identification numbers. 
TABLE 1

SRL Site Code Abbrevlations for Western $1^{\bullet}$ x $2^{\bullet}$ NTMS Quadrangles

Quadrangle

Ade1

Ajo .

Al turas

Baker

Bakersfield

Bond

Boise

Brigham City

Burns

Caliente

Canyon C1ty

Cape Flattery

Cedar City

Challis

Chico

Concrete

Coos Bay

Copalis Beach

Crescent

Duth Valley DV

Delta

E1 Centro

E1ko

Ely

Escalante

Eureka

Flagstaff

Fresno

Goldfield

Grand Canyon

Grangeville
DL

Designator

$\mathrm{AD}$

AJ

AT

BA

BF

BH

BO

$\mathrm{BC}$

$\mathrm{BU}$

CL

$\mathrm{CN}$

$\mathrm{CA}$

CD

$\mathrm{CH}$

CI

$\mathrm{CO}$

CB

CP

CR

DL

EL

EK

EY

ES

EU

FS

FR

GO

GC

GV
Quadrangle

Hatley

Holbrook

Hoquiam

Idaho Fallo

Jordan Valley

JV

Kingman

Klamath Falls

KI

$\mathrm{KF}$

Las Vegas

Long Beach

Los Angeles

Lovelock

Lukeville

Lund

LV

LB

LS

LL

LK

LU

Marble Canýon $\quad M C$

Mariposa MR

McDermLL $\quad$ MT

Medford MF

Mesa MM

Millett

ML.

Needles

Nogales

NE

NO

Okanogan

ON

Pendlet on

PN

Phoenix

Pocatello

$\mathrm{PX}$

PC

Prescott

PR

Price

Pullman

\section{A}

O

Designator

Q

$F$

V

I


TABLE 1 (Continued)

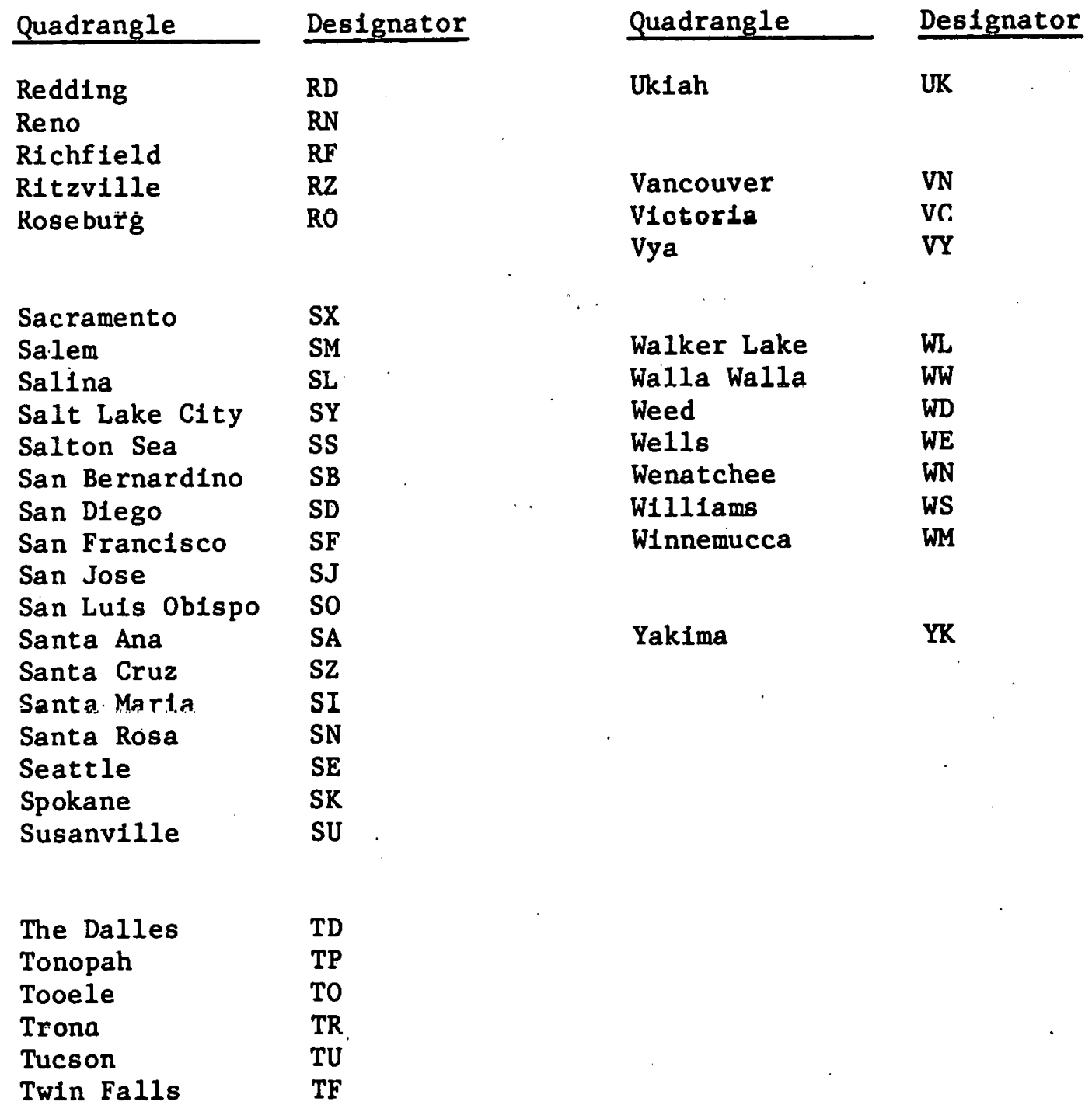




\section{METHODS OF ANALYSIS}

Stream Sediment samples were analyzed for supplemental elements by a contract vendor. Methods of sample dissolution and analysis for each supplemental element are shown in Figure 2. A summary of analytical results for 100 test standards (unknown to the vendor) are shown in Table 2. Analytical results (Table 2) are well within specifications. A standard and blank were analyzed with each group of 40 samples.

Water samples were filtered through a polyrarhonate filter membrane $(0.45 \mu \mathrm{m}$ pore size) and were acidified to a $\mathrm{pH}$ lower than 1.0 with $6 \mathrm{M}$ high-purity nitric acid. The samples were analyzed at the Oak Ridge Gaseous Diffusion Plant by direct-reading emission spectrography and by flameless atomic absorption spectroscopy. The analytical methods are reviewed by Cagle (1977).

\section{DESCRIPTION OF DATA TABLES}

The supplemental data are presented in tabular form in the Appendix. Below is a brief description of the columnar entries.

\section{SRL Identification Number}

Each SRL site code contains 4 letters and a three-digit number. The flrst two letters 1dentify the quadrangle (see "Designator" column in Table 1). MM is the two-letter designator for the Mega $1^{\circ} \times 2^{\circ}$ NTMS quadrangle. The third and fourth letters are taken from the chart below.

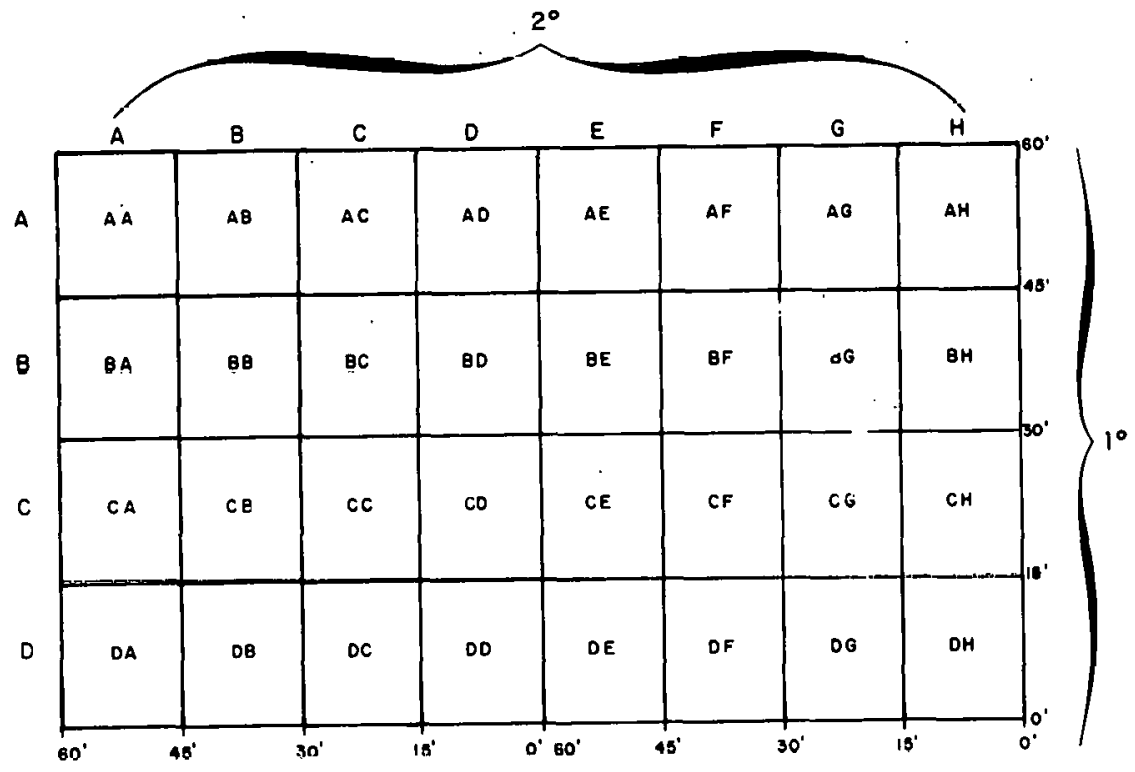




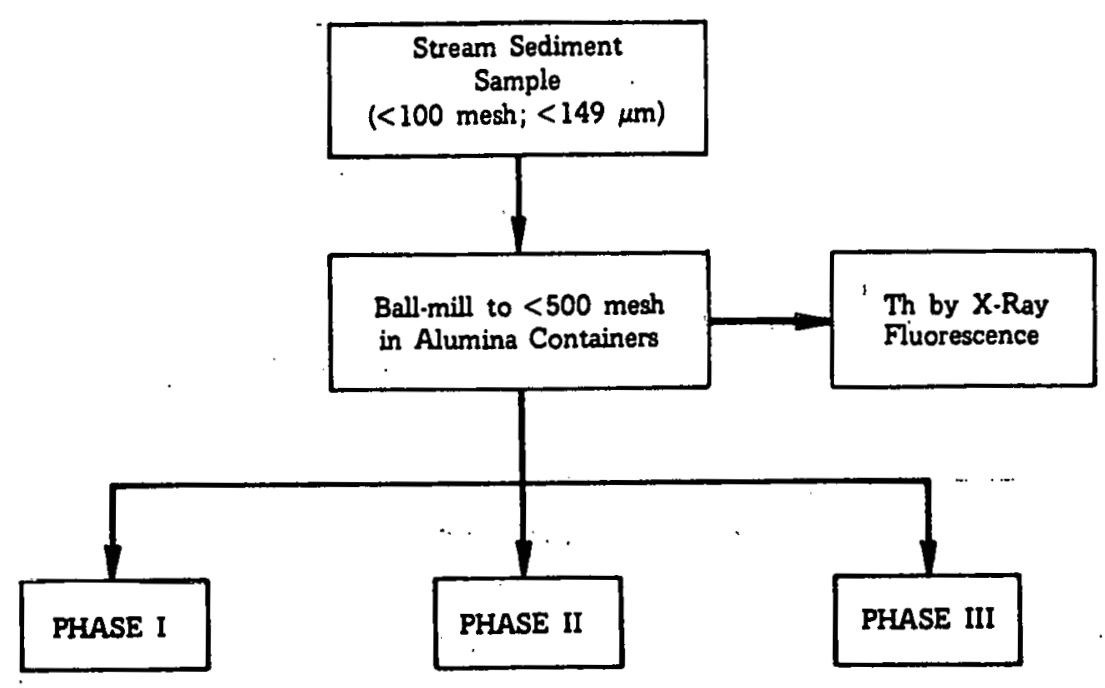

PHASE 1

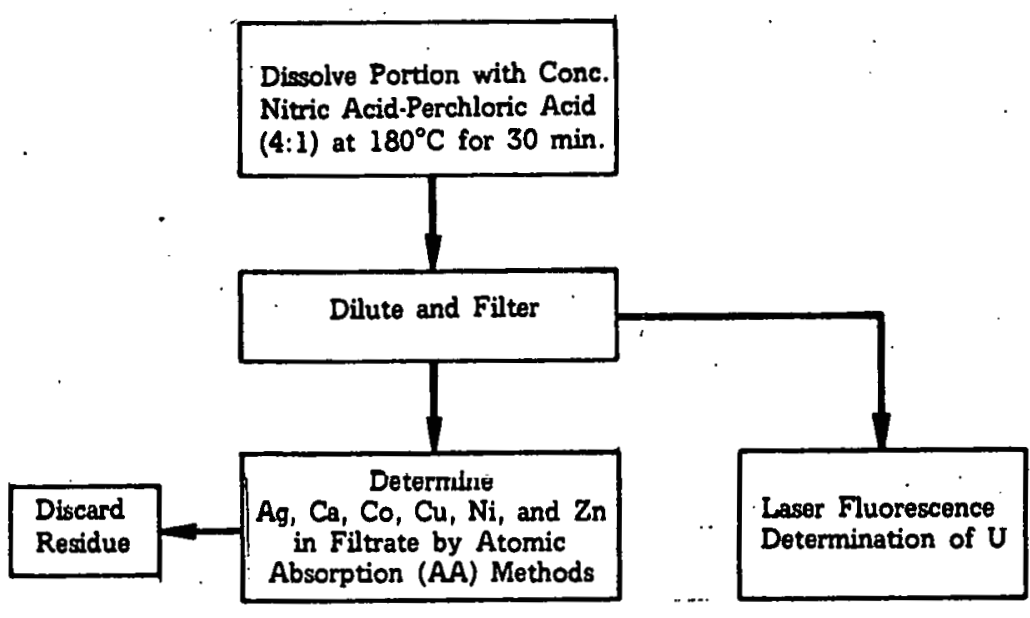

FIGURE 2. Analysis Schere for Supplemental Elements 
PHASE II:

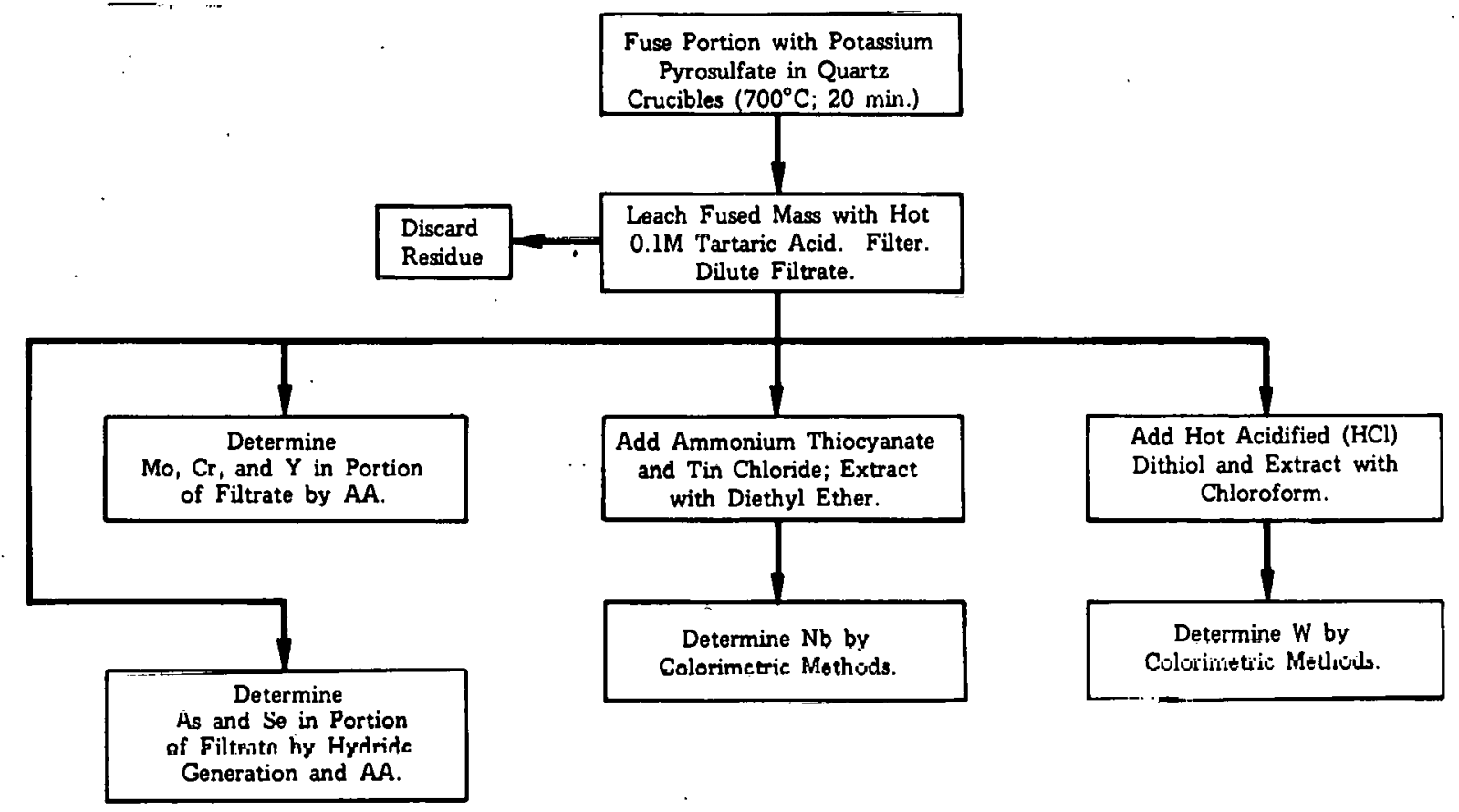

FIGURE 2. Continued 


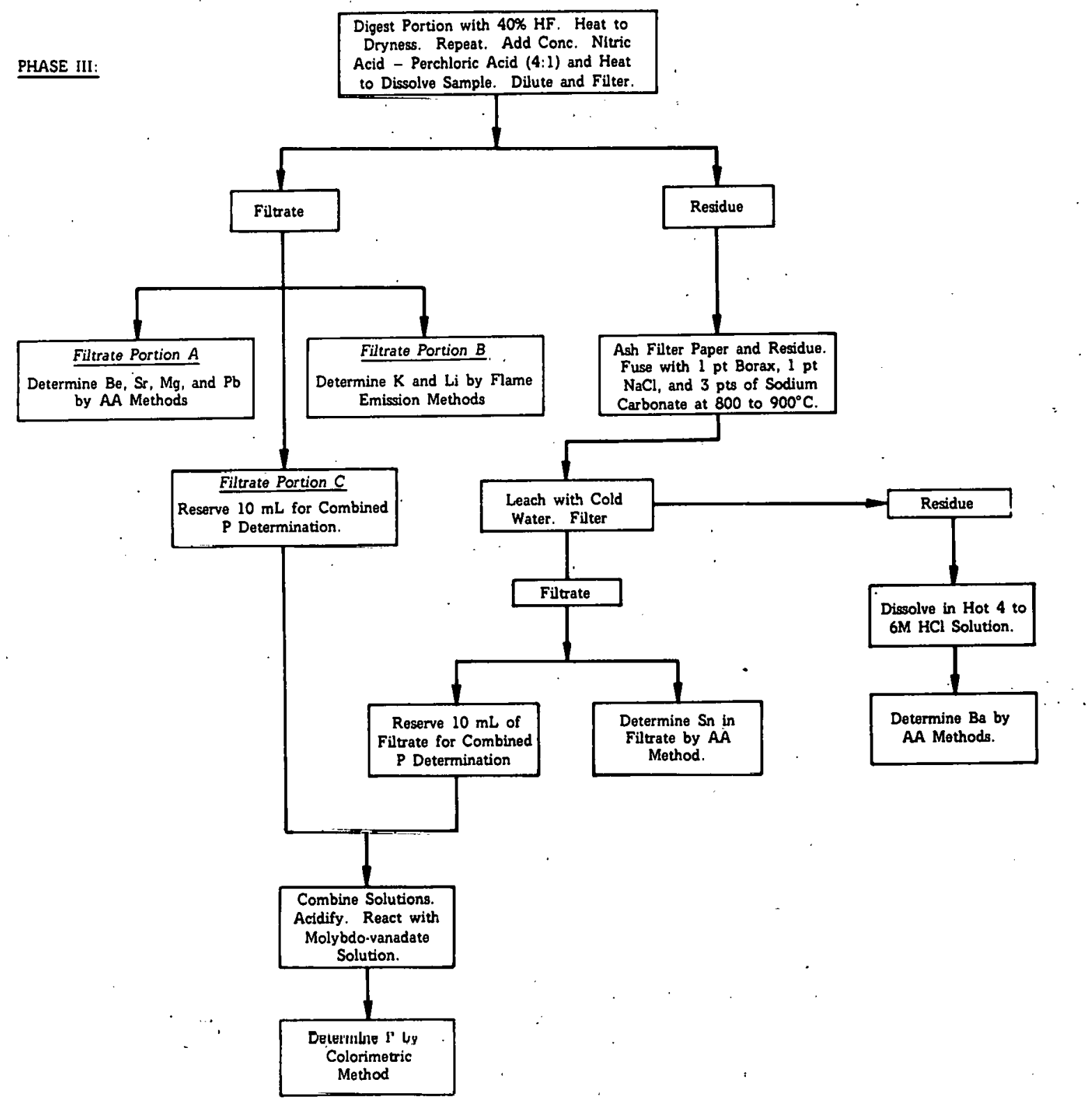

FIGURE 2. Continued 
TABLE 2

Results of Certification Test for Supplementary Analyses*

\begin{tabular}{|c|c|c|c|c|c|}
\hline Element** & $\begin{array}{l}\text { Concentrs } \\
\text { Accepted }\end{array}$ & $\begin{array}{l}\text { 1on, ppm } \\
\text { Measured }\end{array}$ & Diff. & Accuracy, $† \%$ & $\begin{array}{l}\text { Relat1ve } \\
\text { Prec1sion, } \\
(3 \sigma), \% \\
\end{array}$ \\
\hline $\mathrm{Ag}$ & 29.4 & 33.6 & 4.2 & 12 & 5 \\
\hline $\mathrm{Ba}$. & 498 & 508 & 10 & 2 & 6 \\
\hline $\mathrm{Be}$ & 13.6 & 15.1 & 1.5 & 1 & 15 \\
\hline Co & 49.6 & 46.4 & -3.2 & -7 & 10 \\
\hline Cr & 149 & 152 & 3 & 2 & 22 \\
\hline $\mathrm{Cu}$ & 50.1 & 51.1 & 1.0 & 2 & 24 \\
\hline K & 5015 & 5170 & 155 & 3 & 5 \\
\hline Li & 150 & 147 & -3 & -2 & 6 \\
\hline Mg & $4995^{\circ}$ & 5149 & 154 & 3 & 3 \\
\hline Mo & 25 & 25 & 0 & 0 & 12 \\
\hline $\mathrm{Nb}$ & 49.9 & 47.5 & -2.4 & -5 & 27 \\
\hline N1 & 149 & 139 & -10 & -7 & 6 \\
\hline $\mathbf{P}$ & $80 ?$ & 794 & -8 & -1 & 17 \\
\hline $\mathrm{Pl}$ & 308 & 335 & 27 & 12 & 5 \\
\hline $\mathrm{sn}$ & 125 & 121 & -4 & -3 & 14 \\
\hline Sr & 970 & 1169 & 199 & 17 & 10 \\
\hline W & 149.5 & 148 & -1.5 & -1 & 16 \\
\hline $\mathbf{Y}$ & 490 & 434 & -56 & -13 & 12 \\
\hline $\mathrm{Zn}$ & 120 & 112 & -8 & -7 & 4 \\
\hline
\end{tabular}

* Standards included the geochemical soll standards and rock staidards curreully avallable Irum Ũ.S. Geological Survey, SRL stream sediment standards doped with varying levels of the elements, and synthetic standards.

** No statistical data have been collected for $\mathrm{As}, \mathrm{Se}, \mathrm{Ca}$, Th, and extractable U.

$\dagger$ "Accuracy" is a measure of deviation from the accepted value. 
Sites are numbered sequentially within each map unit. Numbers from 001 to 499 designate surface sites. Numbers from 501 to 999 designate ground water sites. The first sediment sample, therefore, taken from the extreme northwestern portion of the Mesa quadrangle would be MMAOOL.

Columns 8 and 9 represent the sample analyzed. In most reports, the $S 1$ fraction [finer than 149 micrometers (U.S. Std. 100 mesh)] was analyzed. S0 and S2 mean that coarser or finer fractions, respectively, were analyzed. Specific fractions will be identified in individual reports as necessary.

DOE Identification Number

Each sample is assigned a DOE ID number. The number consists of 28 characters as follows:

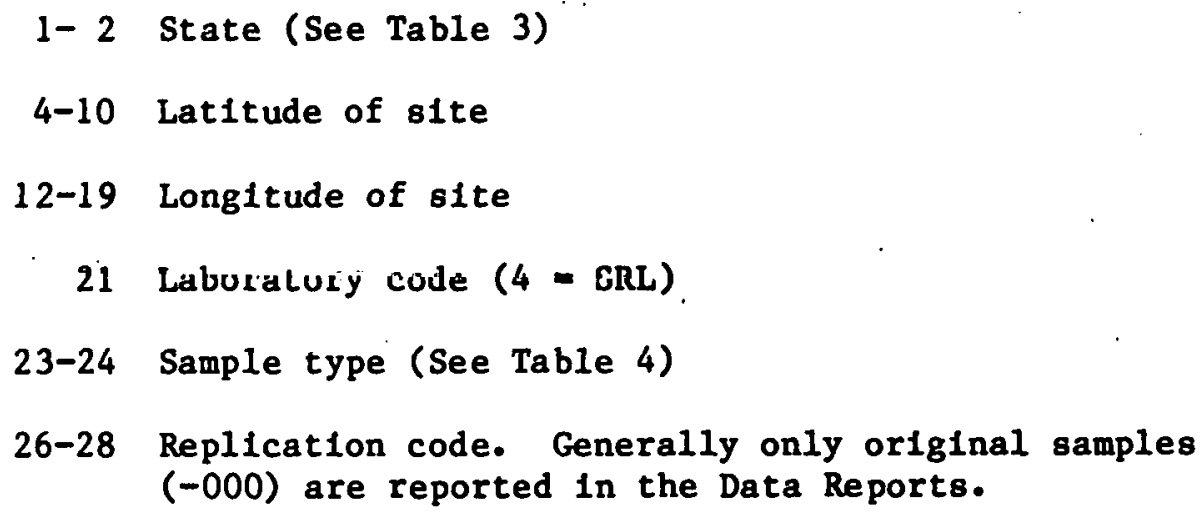

\section{Supplemental Elemental Analyses}

The elements are 11sted alphabelically. Concentrationo of each element are reported in parts per million (ppm) by welght. Values have been rounded to appropriate significant figures. . Note that the elemental (not oxide) concentration is quoted in this table. Values below detection limits are indicated by a minus $(-)$. For example, -3 means that the sample contains less than $3 \mathrm{ppm}$ of that element. If background 1 imit 18 not avallable, a period (.) Is used to Indicate not only that the element was not detected, but that the detection limit is unusually high in that sample. Missing data are indicated by "M." Missing data arc indicative of insufficlent sample for analysis. 
TABLE 3

State Codes Used in DOE Identification Nunber

State

Al abama

Arizona

Arkangas

California

Connecticut

Delaware

District of Columbia

Florida

Georgla

Idaho

Illinois

Ind lana

Kentucky

Loulsiana

Malne

Maryland

Massachusetts

Michlgan

Missigsipp1

Missour1
Code

01

04

05

06

09

10

11

12

13

16

17

18

21

22

23

24

25

26

28

29

\begin{tabular}{ll} 
State & Code \\
\cline { 2 - 2 } Nevada & 32 \\
New Hampshire & 33 \\
New Jersey & 34 \\
New York & 36 \\
North Carolina & 37 \\
& \\
Ohio & 39 \\
Oklahoma & 40 \\
Oregon & 41 \\
Pennsylvania & 42 \\
Rhode Island & 44 \\
& \\
South Carolina & 45 \\
Tennessee & 47 \\
Texas & 48 \\
Utah & 49 \\
Vermonton & 50 \\
V1rglnia & \\
Washington & 51 \\
West V1rg1nia & 53 \\
& 54
\end{tabular}


TABLE 4

Type Codes Used in DOE Sample Identification Number*

Digit Sample Type

50 Stream sediment from flowing stream, sleved $<149 \mu \mathrm{m}$ and dried $\leq 110^{\circ} \mathrm{C}$

51 Stream water, filtered through $\leq 0.8 \mu \mathrm{m}$ filter at the site

52 Well water, filtered through $\leq 0.8 \mu \mathrm{m}$ filter at the site

53 Spring water, filtered through $\leq 0.8 \mu \mathrm{m}$ filter at the site

54 Lake water, filtered through $\leq 0.8 \mu \mathrm{m}$ filter at the site

$55 \quad$ Lake sediment

$56 \quad$ Glacial ti11

$57 \quad$ Peat

58 So11, sieved $>149 \mu \mathrm{m}$ and $<1000 \mu \mathrm{m}$

59 So11, sleved $<149 \mu \mathrm{m}$

60 Stream sediment from dry stream, sleved $>149 \mu \mathrm{m}$ and $<1000 \mu \mathrm{m}$, and dried $\leq 110^{\circ} \mathrm{C}$

61 Stream sediment from dry stream, selved $<149 \mu \mathrm{m}$ and dried $\leq 110^{\circ} \mathrm{C}$.

62 Talus

63 Volcanic neck

64 Playa sediment

65 Hot springs, sinter

66-69 Spare numbers**

* The 28-character DOE-GJO 1dentification number for NURE samples contains two digits to denote sample type. The digits 50 through 69 have been set aside for SRL use.

** To be noted in individual reports as needed. 


\section{REFERENCES}

Cagle, G. W., "The Oak Ridge Analytical Program," pp. 133-156 in Symposium on Hydrogeochemical and Stream Sediment Reconnaissance In the United States. DOE-GJO Report GJBX-77(77).*

SRL-138, Savannah River Laboratory Quarterly and Semtannual Reports, Hydrogeochemtcal and Stream Sediment Reconnaissance, National Uranium Resource Evaluation Program: E. I. du Pont de Nemours \& Co. (Inc.), Savannah River Laboratory, Aiken, S.C.

$\begin{aligned} \text { No. } & \text { Period } \\ 1 & \text { January-March } 1975 \\ 2 & \text { April-June } 1975 \\ 3 & \text { July-September } 1975 \\ 4 & \text { October-December } 1975 \\ 5 & \text { January-March } 1976 \\ 6 & \text { Apr11-June 1976 } \\ 7 & \text { July-September } 1976 \\ 8 & \text { October-December } 1976 \\ 9 & \text { January-March } 1977 \\ 10 & \text { Apr11-June 1977 } \\ 11 & \text { July-September } 1977 \\ 12 & \text { October-December } 1977 \\ 13 & \text { January-March } 1978 \\ 14 & \text { Apri1-September } 1978 \\ 15 & \text { October 1978-March } 1979 \\ 16 & \text { Apr11-September } 1979\end{aligned}$

\begin{tabular}{lll} 
SRL Doc. No. & & $\begin{array}{l}\text { DOE-GJO } \\
\text { Doc. No.* }\end{array}$ \\
\cline { 1 - 2 } DPST-75-138-1 & & GJBX-5(76) \\
DPST-75-138-2 & & GJBX-6(76) \\
DPST-75-138-3 & GJBX-7(76) \\
DPST-75-138-4 & GJBX-8(76) \\
DPST-76-138-1 & GJBX-17(76) \\
DPST-76-138-2 & GJBX-27(76) \\
DPST-76-138-3 & GJBX-63(76) \\
DPST-76-138-4 & GJBX-6(77) \\
DPST-77-138-1 & GJBX-35(77) \\
DPST-77-138-2 & GJBX-55(77) \\
DPST-77-138-3 & GJBX-90(77) \\
DPST-77-138-4 & GJBX-37(78) \\
DPST-78-138-1 & GJBX-66(78) \\
DPST-78-138-2 & GJBX-13(79) \\
DPST-79-138-1 & GJBX-86(79) \\
DPST-79-138-2 & GJBX-160(79)
\end{tabular}

* DOE-GJO reports are available on microfiche from the Grand Juncrion off1ce, DOE, for $\$ 6.00$. Prepaid orders should be sent tó: Bendix Field Engineering Corporation, Technical Library, P. 0. Box 1569, Grand Junction, CO 81501. Checks or money orders should be made out to Bendix Field Engineering Corporation, the operations contractor for DOE's Grand Junction Office. 
I. Sediment Analyses from the Mesa Quadrangle

Data are tabulated for concentrations of extractable uranium and 23 supplementary elements in sediments. The 23 elements are $\mathrm{Ag}, \mathrm{As}, \mathrm{Ba}, \mathrm{Be}, \mathrm{Ca}, \mathrm{Co}, \mathrm{Cr}, \mathrm{Cu}, \mathrm{K}, \mathrm{Ll}, \mathrm{Mg}, \mathrm{Mo}, \mathrm{Nb}, \mathrm{N} 1, \mathrm{P}, \mathrm{Pb}, \mathrm{Se}$, $\mathrm{Sn}, \mathrm{Sr}, \mathrm{Th}, \mathrm{W}, \mathrm{Y}$, and $\mathrm{Zn}$. 
SRL I.D.

MMAAGOISI MMAAOOZSI YMAAO 55 MMAA005SI YMAAODGS: YMAA0075 YMAAO08S YMAAOOSS YMAAOIOSI YMAAOIIS YMAAOICSI YMAAO 14SI MMAAO 15S YMAAO I6S MMAAD 175 YMAAOISSI YMAAOZOS MMAADIS YMAADZS YMAAO23S MMAAO25SI YMAAD265 YMAAD27S MMAADZBS YMAADESSI MAAAOZOSI MMAAD315 MMAAO325 MMAA033S YMAAO35S YMAAD365 MMAA0375 MMAAD3BS1 YMAADBS MMAADYOSI MAAOUOS MMABODIS MMABCOCS YMABOOJS YMABO045 MABO05S MMABO065 YMABO075 MMABO085 YMABO09S YMABOIOS MMAEO 1151 MMABO ICSI YMABOI 35
DOE. I.D.

$04-33.7990-111.9248-4-59-000$ $04-33.8061-111.8907-4-59-000$ $04-33.7987-111.9780-4-59-000$ $04-33$. $7708-1119811-4-59-000$ $04-33.7707-111.9384-4-59-000$ $04-33.7707-11.987-4-59-000$ $04-33.7998-111.8470-4-59-000$ $04-33.7750-111.8470-4-59-000$ $04-33.7750-111.8642-4-59-000$ $04-33.7068-111.8117-4-59-000$ 作 $04-33.7701-111.8139-4-59-000$ $04-33.9097-111.8436-4-59-000$ $04-33.9123-111.8155-4-59-000$ $04-33.9657-111.8240-4-59-000$ $04-33.9626-111.8759-4-59-000$ $04-33.9886-111.9312-4-59-000$ $04-33.9948-111.9782-4-59-000$ $04-33.8404-111.9283-4-59-000$ $04-33.8482-1119031-4-59-000$ $04-33.9884-111.8759-4-59-000$ $04-33.8336-111.8521-4-59-000$ $04-33.8638-111.8468-4-59-000$ $04-33.8477-1118051-4-59-000$ $04-33.8435-111.7741-4-59-000$ $04-33.8136-111.7579-4-59-000$ $04-33.8126-111.7579-4-59-000$ $04-33.8757=111.7675-4-59-000$ $04-33.8322-111.9594-4-59-000$ $04-33.9040-111.7693-4-59-000$ $04-33.8857-111.8035-4-59-000$ $04-33.9823-111.7967-4-59-000$ $04-33.9933-111.8345-4-59-000$ $04-33.9733-111.8663-4-59-000$ $04-33$. $9365-111.8197-4-59-000$ $04-33.8769-111.9086-4-59-000$ $04-33.8772-111.9309-4-59-000$ $04-33.9201-111.9509-4-59-000$ 法 $04-33$. $04-33.9420-111.9507-4-59-000$ $04-33.8779-111.7337-4-59-000$ $04-33.8476-111.6358-4-59-000$ $04-33.8876-111.7033-4-59-000$ $04-33.88720-111.7033-4-59-000$ 04-33.8422-111.6400-4-59-000 04-33.8096-111.6489-4-59-000 04-33.9020-111. $5117-4-59-000$ 04-33.9796-111.7202-4-59-000 04-33.8835-111.5307-4-59-000 $04-33.9$ $04-33.8715-111.5444-4-59-000$ 04-33.9534-111.6936-4 59-000 $04-33.7543-111.5090-4-59-000$

CINCENTRATIONS REPORTED IN WEIGHT PARTS PER MILLION (PPM).

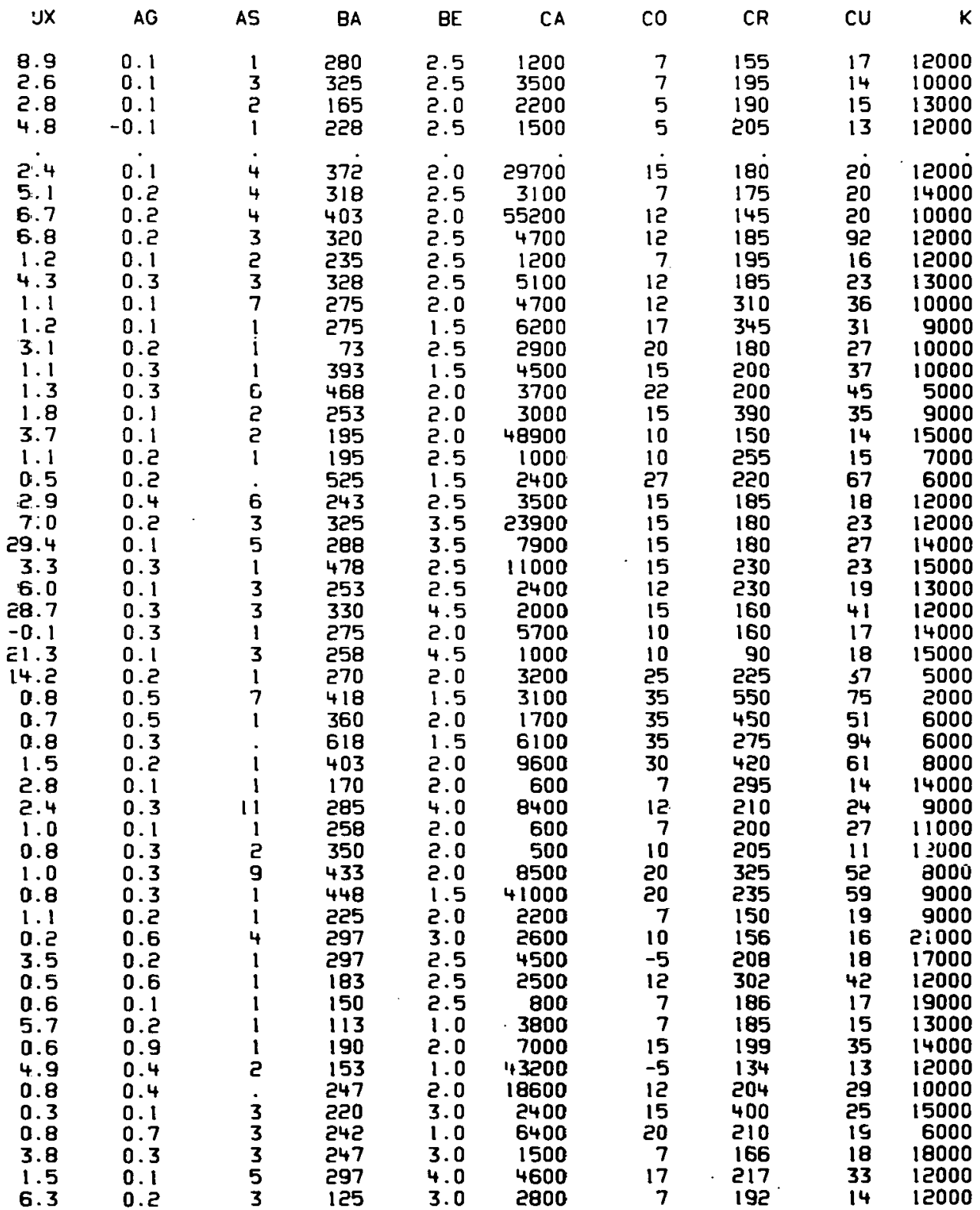


$+\cdots+\cdots$

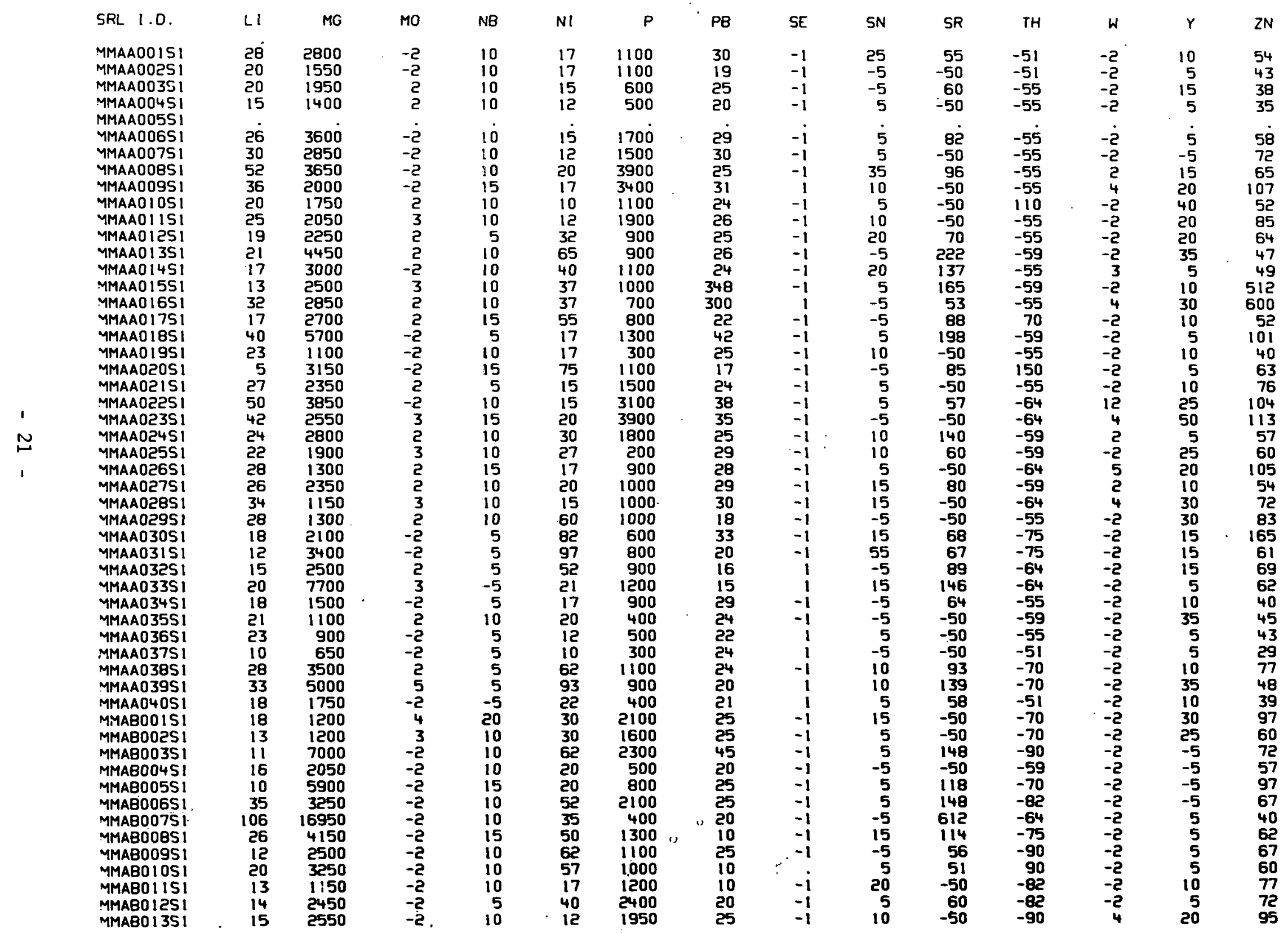

CONCENTRATIONS REPORTED IN WEIGHT PARTS PER MILLION (PPM). 
SRL I.D.

DOE I.D.

YMABO1451 04-33.7518-111:5438-4-59-000 MAA3015S1 04-33.9044-111.6952-4-59-000 $04-33.7674-111.5844-4-59-000$ MMABOI751 $04-33.9070-111.6628-4-59-000$ MMAB01851 $04-33.86 ? 1-111.6265-4-59-000$ MMABOISS1 04-33.7933-111.6934-4-59-000 MMABOZOS1 $04-33.8322-111.7356-4-59-000$ YMAB02151 04-33.8236-111.7243-4-59-000 YMAB022S1 $04-33.7710-111.6661-4-59-000$ YMAB02351 $04-33.7554-111.7079-4-59-000$ MMAB02451 $04-33.7629-111.7272-4-59-000$ MMABO25S1 04-33.8536-111.5588-4-59-000 YMABO2651 $04-33.8434-111.5740-4-59-000$ MMAB02751 04-33.8530-111.5929-4-59-000 YMAB02851 04-33.8153-111.6177-4-59-000 MMABD29S1 04-33.8378-111.5870-4-59-000 YMACODIS1 04-33.9475-111.4385-4-59-000 MMAC00251 04-33.9159-111.3802-4-59. MMACOOS1 04 $0433.9159-111.3602-4-59-000$ YMACO0451 04-33.9175-111.3191-4-59-000 MMAC05S1 04-33.9259-111.3991-4-59-000 MMACOOSS1 $04-33.9259-111.3991-4-59-000$ MMAC00751 $04-33.9047-111.4075-4-59-000$ $\begin{array}{ll}\text { MMACO0751 } & 04-33.9047-111.4075-4-59-000 \\ \text { YMAC005S1 } & 04-33.9695-111.3240-4-59-000\end{array}$ YMAC00651 $04-33.9695-111.3240-4-59-000$ YMAC00951 $04-33.9166-111.4406-4-59-000$ MMACOIOSI 04-33.9916-111.3236-4-59-000 YMAC01151 04-33.9110-111.4825-4-59-000 MMACO12S1 04-33.9910-111.3713-4-59-000 YMACO1351 04-33:8847-111.4759-4-59-000 YMACO14SI $04-33.9714-111.3902-4-59-000$ YMAC015S1 04-33.8781-111.4395-4-59-000 YMAC01651 04-33.8772-111.3159-4-59-000 MMAC01751 04-33.8835-111.4043-4-59-000 MMAC01851 04-33.8862-111.3631-4-59-000 MMACO19S1 04-33.9420-111.4819-4-59-000 MMACO20S1 04-33.7607-111.4953-4-59-000 MMACOZISI $04-33.9649-111.4813-4-59-000$ MMACO22S! $04-33.7973-111.4864-4-59-000$ MMAC02351 04-33.9731-111. 4402-4-59-000 MMAC0245i $04-33.8067-111.4457-4-59-000$ MMAC025S1 04-33.8E96-111.3421-4-59-000 MMACC26S1 04-33.8364-1111.4715-4-59-000 MMACO275) 04-33.8E91-111.3143-4-59-000 MMACO2BS1 $04-33.8452-111.4414-4-59-000$ MACO29S1 $04-33.8415-111.3056-4-59-000$ 4MACO30S1 $04-33.8540-111.4149-4-59-000$ MAC03151 04-33.8 $=62-111.2958-4-59-000$ MAC $315104-33.8265-111.2958-4-59-000$ MMACO3ESI $04-33.8062-111.2961-4-59-000$ MMACO3351 04-33.8040-111.2730-4-59-000 MMACO3451 $04-33.7=07-111.2651-4-59-000$ MMACO35S1 $04-33.7 E 27-1111.2809-4$ 59-000 MMAC 3651 ( $04-33.7529-111.3084-4-59-000$

CONCENTRATIONS REPORTED IN WEIGHT PARTS PER MILLION (PPM).

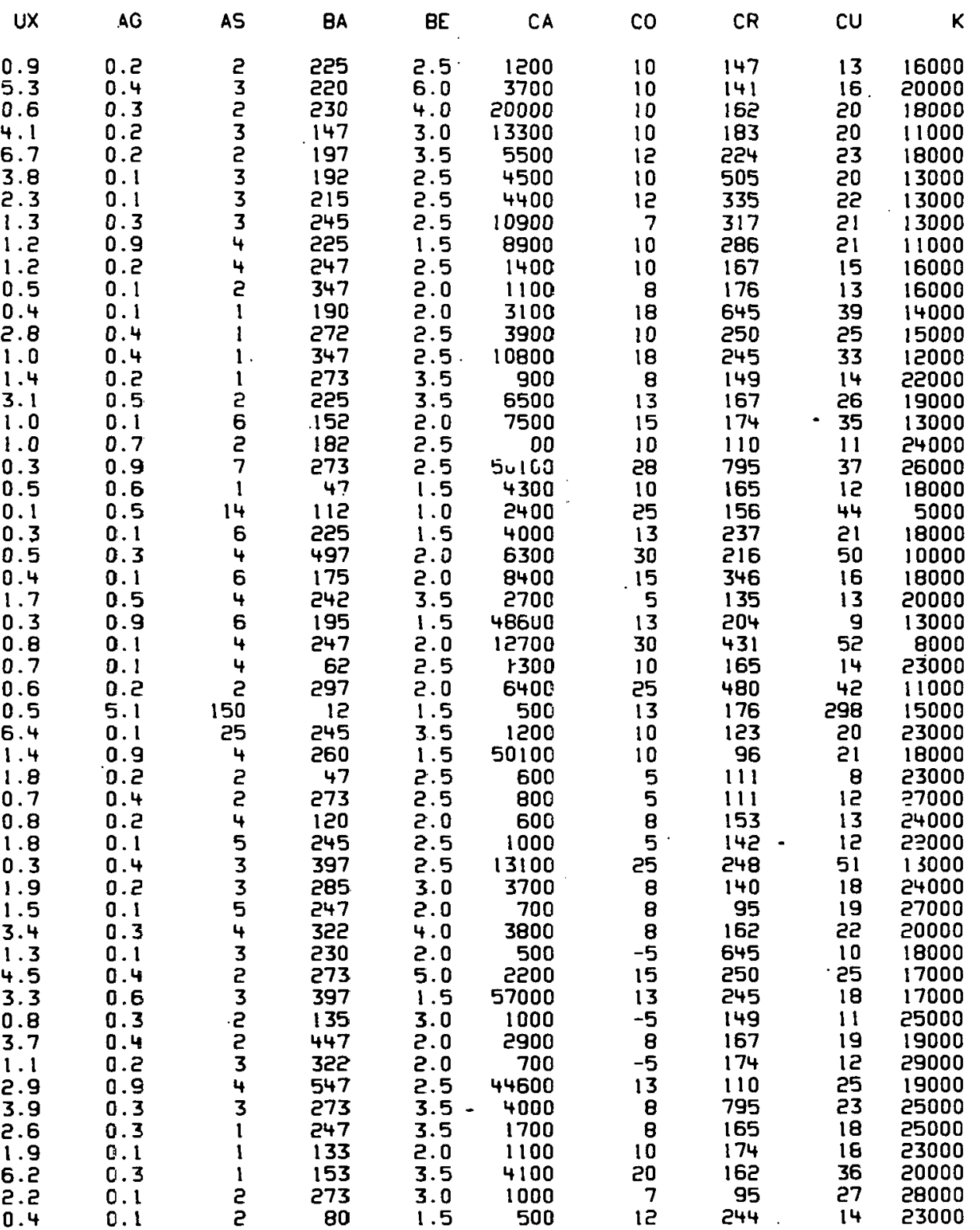


$\ldots$

MMABO 1451 MMABO I5S

MMABO I6S

MMABO 175

MMABO 185

MMABOISS

MMABOSOSI

MMABO2ZS

MMABO235

MMABO245I

MMAB025S

MMABO265

MMAB0275I

MMAB02BSI

MABO29S

MMACOOZS

YMAC 0035

MMAC 0045

MMAC005SI

MMAC006SI

MMAC0075

MMACOOBS

MMACOIOSI

MMACOIOSI

MMACO 115

MMACO12S

MMACO 145

MMACO 1551

MMACO 1651

MMACO 1751

MMACO 1851

MMACO 1951

MMAC02051

MMACO21S1

MMACO2ZS

MMAC 02351

MMAC 25451

MMAC 0255

MMAC0265

MMAC 02851

MMAC 029S1

MMAC 03051

MMAC0315

MMACO325

MMAC0335

MMACO34S

MMAC0355

MMAC03651

MMACO375:

CONCENTRATIONS REPORTED IN WEIGHT PARTS PER MILLION (PPM).

$\begin{array}{rr}L 1 & M G \\ 17 & 1100 \\ 35 & 2900 \\ 24 & 2750 \\ 16 & 2300 \\ 24 & 1400 \\ 15 & 1400 \\ 11 & 3750 \\ 10 & 3400 \\ 16 & 3850 \\ 16 & 1050 \\ 15 & 2450 \\ 6 & 5400 \\ 16 & 2900 \\ 12 & 4250 \\ 15 & 2300 \\ 21 & 3100 \\ 22 & 3350 \\ 13 & 1950 \\ 20 & 8500 \\ 16 & 5100 \\ 16 & 3000 \\ 10 & 3200 \\ 15 & 3150 \\ 12 & 3150 \\ 29 & 3000 \\ 9 & 3650 \\ 56 & 5500 \\ 14 & 3000 \\ 8 & 4800 \\ 11 & 2950 \\ 17 & 2850 \\ 22 & 5600 \\ 14 & 2250 \\ 8 & 2150 \\ 16 & 2350 \\ 13 & 2350 \\ 39 & 3000 \\ 16 & 3150 \\ 34 & 3050 \\ 22 & 3000 \\ 11 & 2050 \\ 16 & 3150 \\ 44 & 7000 \\ 14 & 2400 \\ 17 & 2650 \\ 12 & 2300 \\ 47 & 4350 \\ 17 & 3300 \\ 16 & 2500 \\ 17 & 2800 \\ 21 & 3450 \\ 27 & 600 \\ 20 & 3000 \\ & \\ 15\end{array}$

$\begin{array}{rr}M O & N B \\ & \\ -2 & 5 \\ -2 & 5 \\ 2 & 10 \\ -2 & 5 \\ -2 & 5 \\ -2 & 15 \\ -2 & 10 \\ -2 & 10 \\ -2 & 15 \\ -2 & 10 \\ -2 & 10 \\ -2 & 15 \\ -2 & 15 \\ -2 & 15 \\ -2 & 15 \\ -2 & 15 \\ -2 & 10 \\ -2 & 10 \\ -2 & 15 \\ -2 & 10 \\ -2 & 5 \\ -2 & 5 \\ -2 & 10 \\ -2 & 10 \\ -2 & 10 \\ 2 & 15 \\ -2 & 10 \\ 2 & 15 \\ -2 & 10 \\ -2 & 10 \\ -2 & 10 \\ -2 & 15 \\ -2 & 5 \\ -2 & 15 \\ -2 & 15 \\ -2 & 15 \\ -2 & 10 \\ 2 & 5 \\ -2 & 10 \\ -2 & 10 \\ -2 & 15 \\ -2 & 15 \\ -2 & 15 \\ -2 & 5 \\ -2 & 10 \\ -2 & 5 \\ -2 & 15 \\ 2 & 10 \\ -2 & 15 \\ -2 & 10 \\ -2 & 10 \\ 3 & 15 \\ -2 & 10 \\ -2 & 10\end{array}$

$\begin{array}{rr}N 1 & P \\ & \\ 20 & 400 \\ 15 & 2800 \\ 40 & 1300 \\ 32 & 1600 \\ 20 & 2400 \\ 35 & 1600 \\ 37 & 1600 \\ 40 & 1500 \\ 55 & 1200 \\ 18 & 900 \\ 20 & 1000 \\ 83 & 2100 \\ 30 & 2300 \\ 48 & 1600 \\ 13 & 300 \\ 20 & 2100 \\ 25 & 500 \\ 15 & 200 \\ 208 & 600 \\ 30 & 300 \\ 15 & 300 \\ 40 & 1900 \\ 43 & 900 \\ 38 & 950 \\ 18 & 700 \\ 40 & 1800 \\ 190 & 2400 \\ 18 & 1200 \\ 195 & 1400 \\ 18 & 150 \\ 10 & 350 \\ 23 & 400 \\ 8 & 300 \\ 13 & 200 \\ 18 & 300 \\ 8 & 300 \\ 45 & 900 \\ 13 & 1900 \\ 13 & 300 \\ 15 & 1700 \\ 13 & 300 \\ 18 & 400 \\ 35 & 300 \\ 18 & 800 \\ 13 & 700 \\ 28 & 450 \\ 15 & 700 \\ 18 & 1700 \\ 18 & 1200 \\ 17 & 500 \\ 17 & 2500 \\ 7 & 500 \\ 22 & 300 \\ & \\ 19\end{array}$

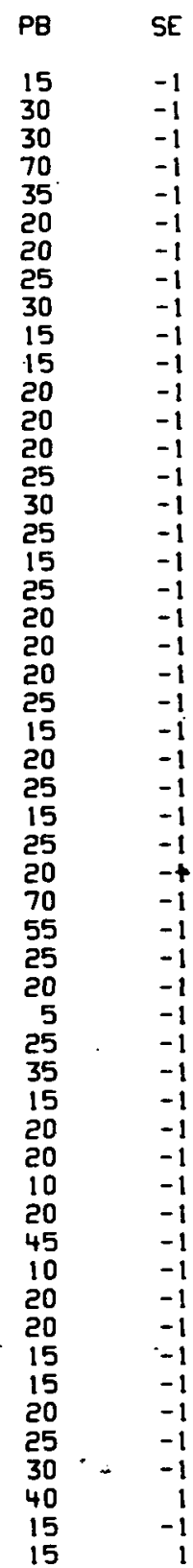

$\begin{array}{rr}\text { SE } & \text { SN } \\ -1 & -5 \\ -1 & 5 \\ -1 & 15 \\ -1 & -5 \\ -1 & 5 \\ -1 & -5 \\ -1 & 15 \\ -1 & 15 \\ -1 & -5 \\ -1 & 5 \\ -1 & -5 \\ -1 & -5 \\ -1 & -5 \\ -1 & 15 \\ -1 & 5 \\ -1 & 5 \\ -1 & -5 \\ -1 & -5 \\ -1 & 5 \\ -1 & 10 \\ -1 & 5 \\ -1 & 10 \\ -1 & -5 \\ -1 & -5 \\ -1 & -5 \\ -1 & 5 \\ -1 & 5 \\ -1 & 10 \\ -1 & -5 \\ -1 & 15 \\ -1 & -5 \\ -1 & 5 \\ -1 & -5 \\ -1 & -5 \\ -1 & 10 \\ -1 & -5 \\ -1 & -5 \\ -1 & 10 \\ -1 & 5 \\ -1 & -5 \\ -1 & 15 \\ -1 & -5 \\ -1 & 5 \\ -1 & -5 \\ -1 & 5 \\ -1 & -5 \\ -1 & -5 \\ -1 & 10 \\ -1 & -5 \\ -1 & 5 \\ 1 & -5 \\ -1 & -5 \\ 1 & -5 \\ & \end{array}$

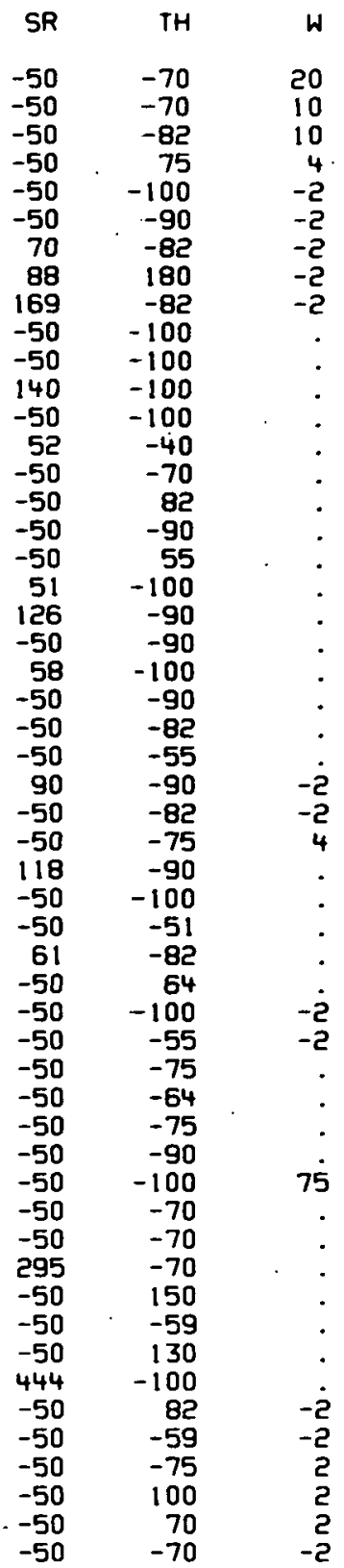

6120 
SRL $1 . D$.

DOE I.D.

MMACU3851 $04-33.8334-111.2756-4-59-000$ MALDOO151 04-33.7759-111.1430-4-59-000. YMADO02S1 $04-33.8343-111.2250-4-59-000$ MMAD00351 04-33.7735-111.1894-4-59-000 MMAD004S1 04-33.8334-111.2261-4-59-000 MMADO05S1 04-33.7768-111.2115-4-59-000 MMADO06S $1 \quad 04-33.8691-111.2139-4-59-000$ MMAD00751 04-33.7674-111.0294-4-59-000 MMAD008S1 $04-33.8867-111.2011-4-59-000$ MMAD009S1 $04-33.7865-111.0251-4-59-000$ MMADOIOS1 $04-33.9090-111.2202-4-59-000$ YMAD01151 04-33.7690-111.0491-4-59-000 YMADO12S1 04-33.9182-111.1942-4-59-000 MMAD01351 04-33.8056-111.0637-4-59-000 YMAD014S1 04-33.9421-111.1681-4-59-000 MMADO15SI 04-33.8226-111.0659-4-59-000 MMAD016S1 04-33.9472-111.0193-4-59-000 MMAD01751 04-33.8262-111.0330-4-59-000 MMAD01851 04-33.9678-111.0565-4-59-000 YMAD01951 04-33.7733-111.0838-4-59-000 MMADOZOS1 04-33.9210-111.0514-4-59-000 MMADODIS $04-33.7876-111.1810-4-59-000$ YMADO2วS1 04-33.9465-111.0586-4-59-000 MMADO23S1 04-33.7970-111.1632-4-59-000 MMAD024SI 04-33.9068-111.1177-4-59-000 MMAD025S1 $04-33.8924-111.1078-4-59-000$ MMAD026S1 04-33.8822-111.1459-4-59-000 MMAD02751 04-33.8957-111.1290-4-59-000 MMAD028S1 04-33.9550-111.1549-4-59-000 YMAD029S1 04-33.9722-111.1373-4-59-000 MMADO30S1 $04-33.9817-111.1204-4-59-000$ MMAD031S1 04-33.9349-111.0979-4-59-000 MMAEDO1S1 04-33.7705-110.9456-4-59-000 MMAEO02S1 04-33.7962-110.9822-4-59-000 MMAE00351 04-33.8369-110.9781-4-59-000 YMAE00451 04-33.8181-110.9300-4-59-000 YMAEOO5SI MMAEOOSI $04-33.8393-110.9557-$ MMEEOB51 04-33.7802-110. MMEE00751 04-33.8674-110.9245-4-59-000 MMAEOOSI 04-33.8619-110.9434-4-59-000 MMAEOOSSI 04-33.8705-110.9751-4-59-000 MMAECIOSI 04-33.9000-110.9503-4-59-000 MMAEOIISI 04-33.9266-110.9607-4-59-000 MMAEOILSI 04-33.9584-110.9897-4-59-000 MMAEOI3SI 04-33.9823-110.9705-4-59-000 MMAE014SI 04-33.9851-110.9477-4-59-000 MMAE0ISSI $04-33.9376-110.9260-4-59-000$ MMAEOI6S1 $04-33.9229-110.9155-4-5$ MMAE01751 04-33.8981-110.9057-4-59-000 MMAEOI8S1 $04-33.8713-110.8853-4-59-000$ MMAE0ISSI 04-33.8519-110.8700-4-59-000 MMAEOZOSI $04-33.8231-110.8565-4-59-000$ MMAED21S1 $04-33.7760-110.7962-4-59-000$
CONCENTRATIONS REPORTED IN WEIGHT PARTS PER MILLION (PPM).

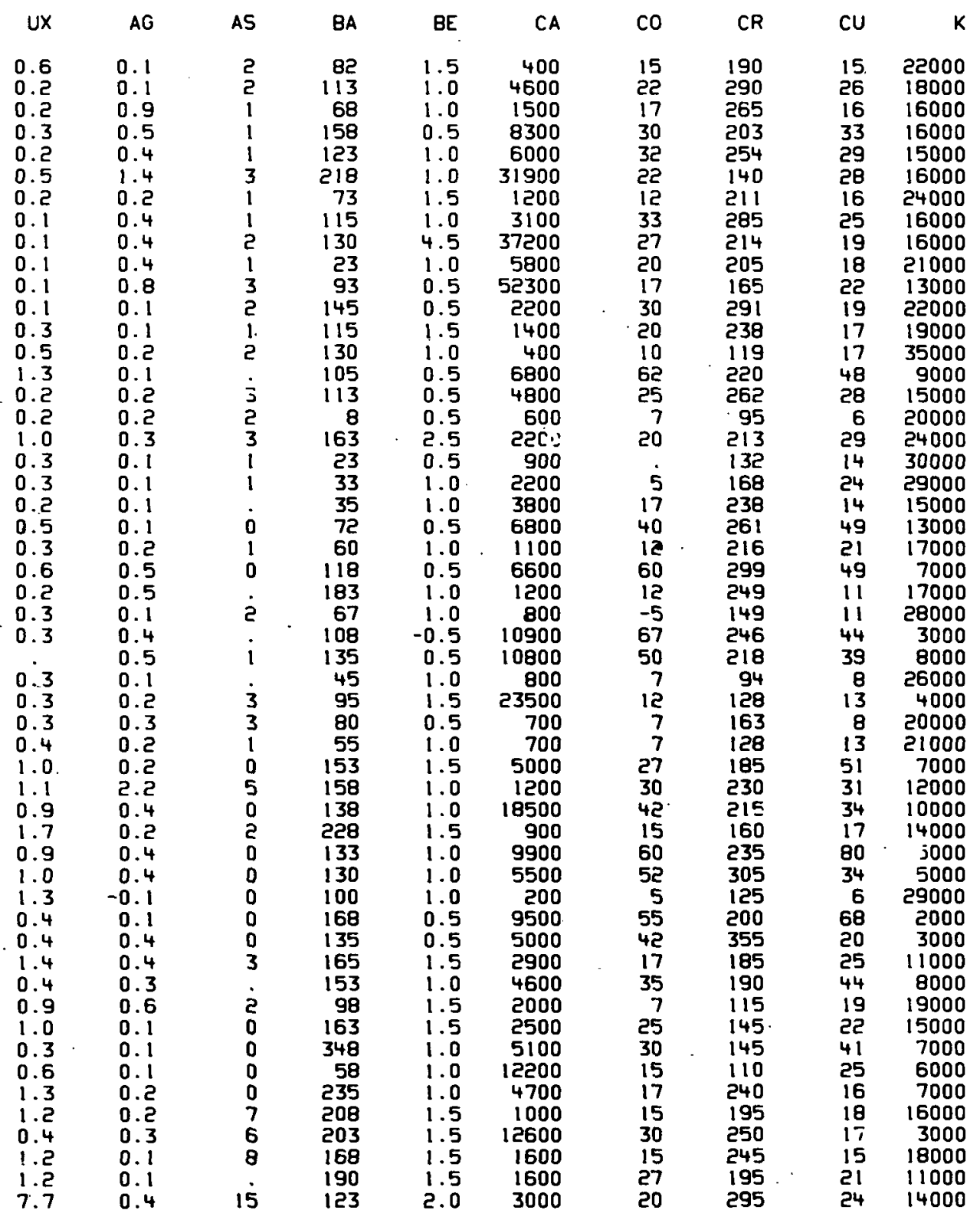


$\ldots$

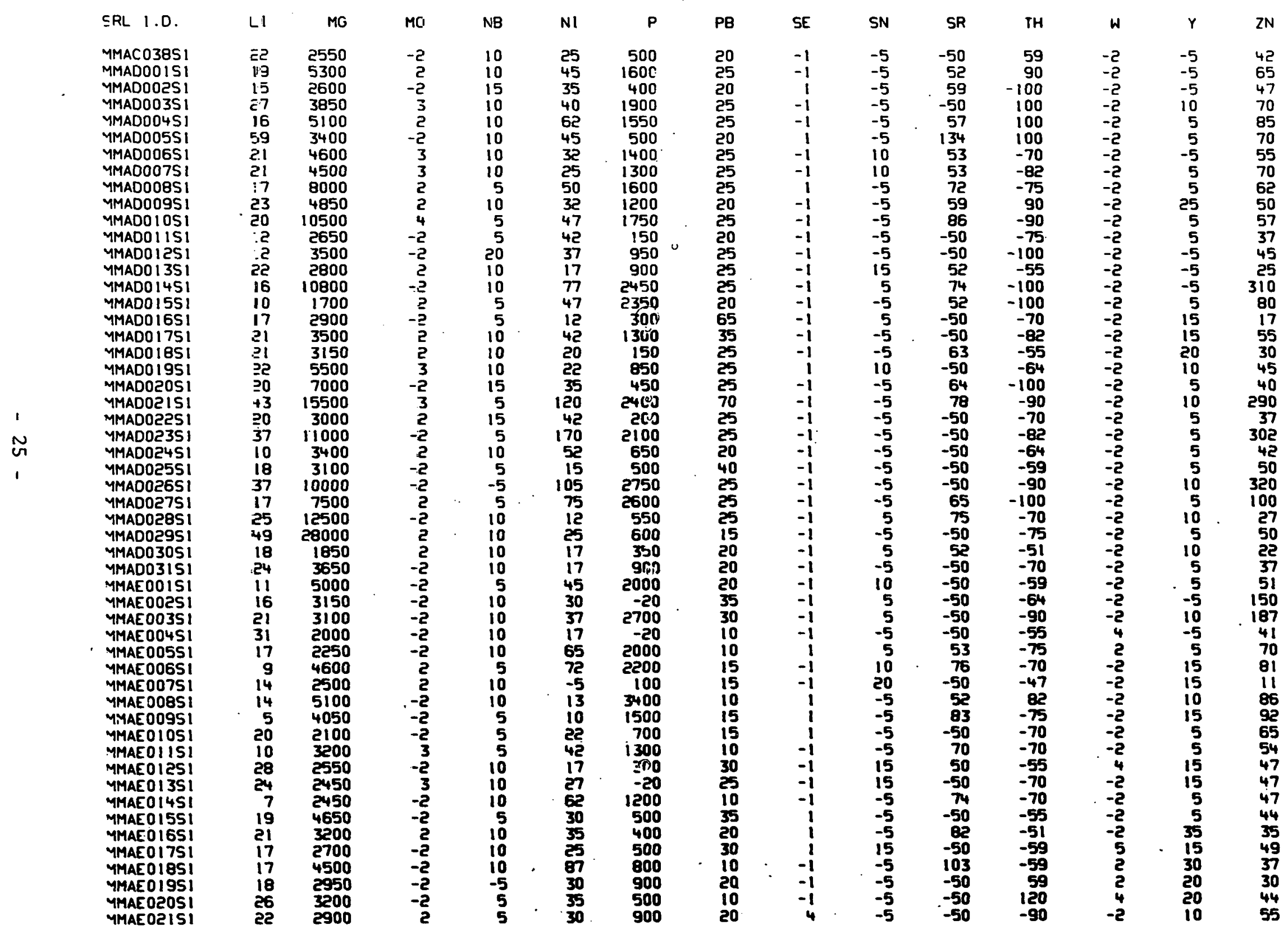

CONCENTRATIONS REPORTED IN WEIGHT PARTS PER MILLION (PPM). 
$\ldots$

DOE I.D.

SRL I.D.

MMAE02251 $04-33.7968-110.8303-4-59-000$ VMAL $02351 \quad 04-33.7977-110.8007-4-59-000$ YMAE024S1 $04-33.7512-110.7813-4-59-000$ MMAE025S1 $04-33.3401-110.8017-4-59-000$ MMAE026S1 $04-33.3642-110.8109-4-59-000$ MMAE 027S1. 04-33. 3481-110.7986-4-59-000 MMAEO2BSI $04-33.9742-110.8062-4-59-000$ MMAE029S1 $04-33.9111-110.8217-4-59-000$ YMAE030S1 04-33.3891-110.8421-4-59-000 $04-33.5252-111.8570-4-59-000$ -33.5583-111. 8555-4-59-000 (0.351 04-33.5857-111.8564-4-59-000 YMBA00451 04-33.5056-111.8461-4-59-000 MMBA00551 04-33. 2882-111.8127-4-59-000 4MBA00651 04-33.5095-111.8045-4-59-000 4MBA00751 04-33.5789-11. $7596-4-59-000$ MMBAO0851 04-33.5632-111.7698-4-59-000 YMBAOOSI 04-33.5563-111. MBAOSS1 04-33.5563-111.8084-4-59-000 4MBAOIOSI 04-33.5318-111.8211-4-59-000 4MBAOIISI 04-33.514-1-111.7747-4-59-000 MMBAOLICSI 04-33.5154-111.7748-4-59-000 MMBAOI3S1 04-33.5021-111.8854-4-59-000 MMBA014S1 $04-33.5376-111.8891-4-59-000$ MMBAD 15S1 $04-33.5630-111.8898-4-59-000$

CONCENTRATIONS REPOFTED IN WEIGHT PARTS PER MILLION (PPM).

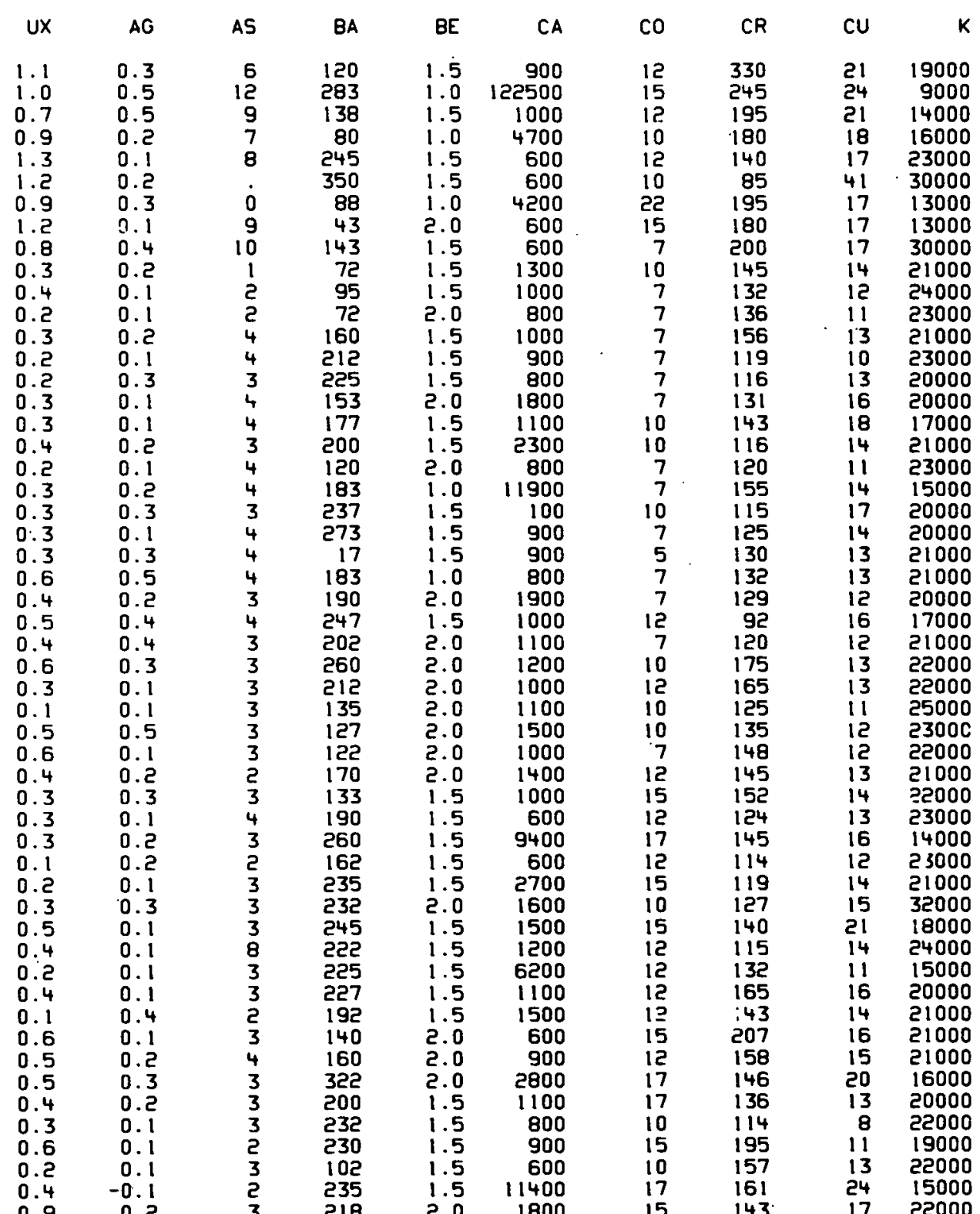


...

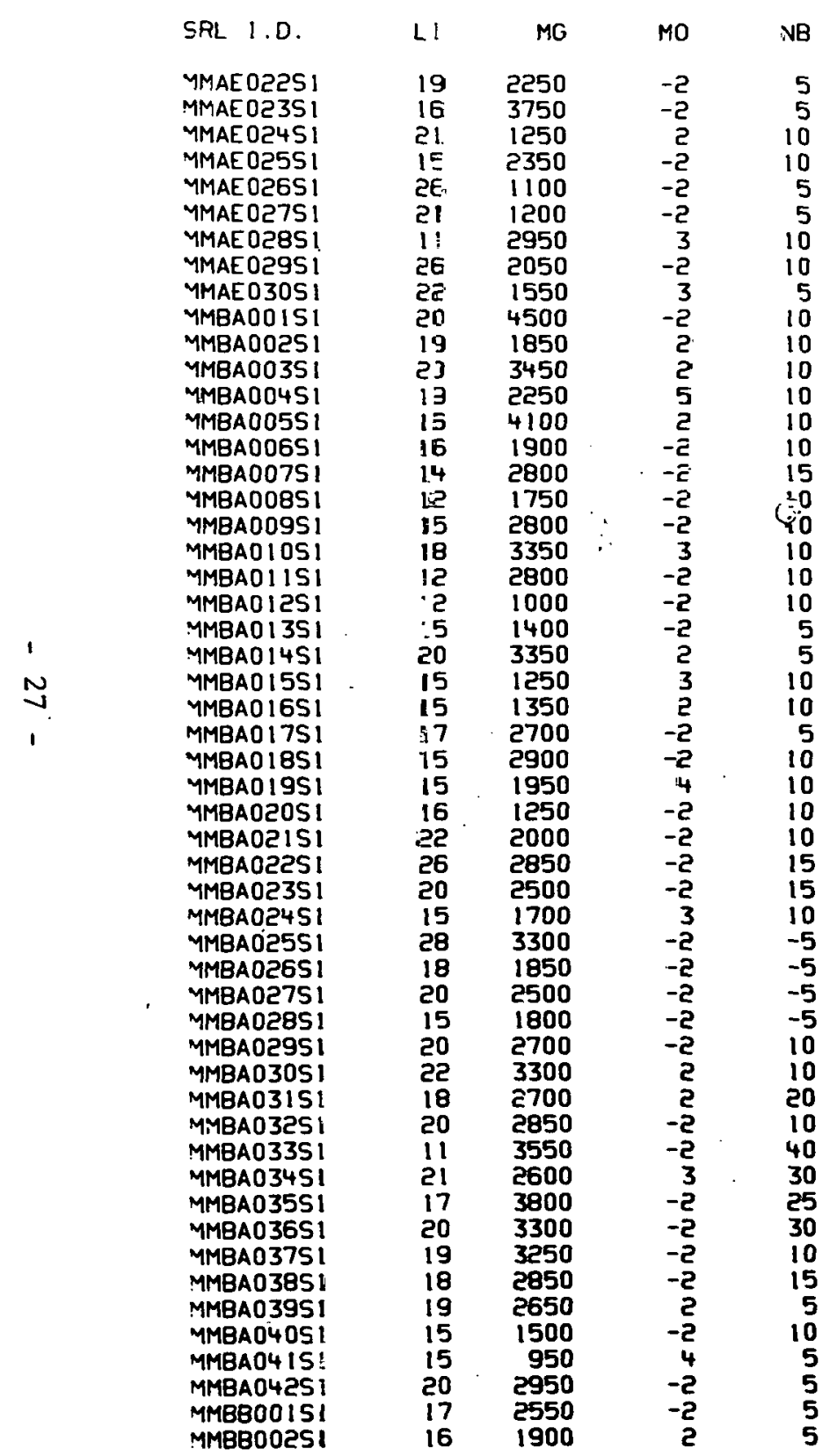

CONCENTRATIONS REPORTED IN WEIGHT PARTS PER MILLIDN (PPM).

N1
27
27
17
15
12
17
37
22
12
27
20
20
17
10
15
15
20
15
17
20
15
17
12
22
17
17
17
15
17
17
17
15
15
22
15
22
15
22
22
30
20
15
17
22
15
17
47
20
12
17
12
27
22

$\begin{array}{rrrr}P & \text { PB } & \text { SE } & \text { SN } \\ 500 & 15 & -1 & 5 \\ 700 & 20 & 1 & -5 \\ 400 & -10 & -1 & -5 \\ 1400 & 20 & -1 & 15 \\ -20 & 20 & 1 & -5 \\ 120 & -10 & -1 & 5 \\ 2320 & 20 & -1 & 15 \\ -20 & 20 & -1 & -5 \\ 400 & 20 & -1 & -5 \\ 400 & 20 & -1 & 5 \\ 700 & 20 & -1 & 5 \\ 700 & 25 & -1 & -5 \\ 700 & 20 & -1 & -5 \\ 400 & 170 & -1 & 5 \\ 400 & 45 & -1 & -5 \\ 500 & 130 & -1 & 15 \\ 500 & 30 & -1 & 5 \\ 500 & 25 & -1 & -5 \\ 400 & 25 & -1 & -5 \\ 500 & 15 & -1 & -5 \\ 500 & 25 & -1 & -5 \\ 500 & 20 & -1 & -5 \\ 400 & 25 & -1 & 5 \\ 1000 & 30 & -1 & 10 \\ 1000 & 20 & -1 & -5 \\ 1200 & 20 & -1 & -5 \\ 1000 & 30 & -1 & -5 \\ 1200 & 25 & -1 & -5 \\ 800 & 20 & -1 & -5 \\ 1000 & 25 & -1 & -5 \\ 1200 & 15 & -1 & 5 \\ 1000 & 15 & -1 & 10 \\ 1100 & 35 & -1 & -5 \\ 800 & 25 & -1 & 10 \\ 600 & 20 & -1 & -5 \\ 500 & 20 & -1 & -5 \\ 400 & 15 & -1 & -5 \\ 700 & 20 & -1 & -5 \\ 700 & 30 & -1 & -5 \\ 700 & 20 & -1 & -5 \\ 800 & 20 & -1 & -5 \\ 600 & 25 & -1 & -5 \\ 500 & 25 & -1 & -5 \\ 1000 & 20 & -1 & 10 \\ 800 & 40 & -1 & -5 \\ 1000 & 25 & -1 & -5 \\ 1500 & 15 & -1 & -5 \\ 1200 & 20 & -1 & -5 \\ 300 & 25 & -1 & -5 \\ 1000 & 25 & -1 & -5 \\ 300 & 25 & -1 & -5 \\ 1800 & 25 & -1 & -5 \\ 1600 & 25 & -1 & -5 \\ & & -5\end{array}$

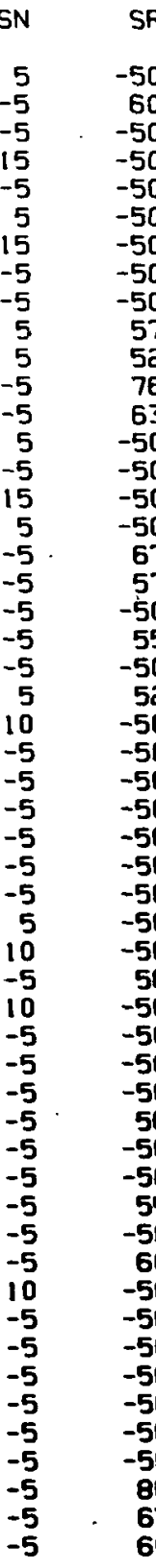

$S R$
-50
60
-50
-50
-50
-50
-50
-50
-50
57
52
76
63
-50
-50
-50
-50
67
57
-50
55
-50
52
-50
-50
-50
-50
-50
-50
-50
-50
-50
58
-50
-50
-50
-50
56
-50
-50
54
-50
60
-50
-50
-50
-50
-50
-50
-50
80
67
60

$T H$
-75
-82
-55
-55
55
-55
-75
-51
-55
-55
-59
59
-55
-55
-50
-82
-75
-75
-55
75
-59
-70
-70
-70
-55
-59
90
-82
64
-75
-59
-100
-82
-75
-75
-90
-70
-90
-100
-100
-82
-82
-82
-82
-100
-100
-100
-100
-100
-82
-100
-100
-90 
$\cdots \cdots$

SRL I.D. DOE 1.0 .

YMBEũ03S1 $04-33.5466-111.6427-4-59-000$ :MB3004S1 04-33.5515-111.6062-4-59-000 MMBB005S1 04-33.5420-111.5779-4-59-000 MMBB005S1 $04-33.5250-111.5755-4-59-000$ MMBB007S1 $04-33.5245-111.6150-4-59-000$ 4MBB008SI $04-33.6 ! 92-111.7192-4-59-000$ 4MBB009S1 $04-33.5883-111.7341-4-59-000$ 4MBB0 IDS1 $04-33.6222-111.6813-4-59-000$ 4MBBOLISI 04-33.5303-111. 7309-4-59-000 $04-33.5516-111.73199-4-59-000$ $04-33.5874-111.6785-4-59-000$ 40.901451 YMBBO15S1 MMBBOIGS1 04-33.5873-111.6380-4-59-000 YMBBO 251 04-33.6337-111.6508-4-59-000 MMBBOIBSI 04-33.6685-111.6693-4-59-000 MMBBOLIS1 04-33.7001-11!.6710-4-59-000 4MBBOCLSI $04-33.7420-111.6901-4-59-000$ पMBBOelS1 04-33.7419-111.7348-4-59-000 04-33.6905-111.7174-4-59-000 04-33.6600-111. $7145-4-59-000$ MMBB025S1 04-33.6638-111.5619-4-59-000 MMBB026S1 04-33.6604-111.5917-4-59-000 4MBB027S1 04-33.6307-1111.5876-4-59-000 MMBB0285 $\quad 04-33.7322-111.5123-4-59-000$ 4MBB02951 04-33:6945-111.5419-4-59-000 4MBB03051 04-33.6120-111.5531-4-59-000 MMBB03151 04-33.5979-111.5497-4-59-000 4MBB03251 04-33.5791-111. 5149-44-59-000 4MBB23351 04-33.5621-111.5392-4-59-000 4MBB03451 04-33.6371-:11.5144-4-59-000 MMBBO35S1 MMBO3751 $04-33$. MMBBO3751 $04-3$ 4MBB03851 04-33.7370-111.5751-4-59-000 4MBB 3951 04-33.7108-111.5832-4-59-000 MMBD40S1 04-33.7103-111.6153-4-59-000 MMBCDOIS1 04-33.5356-111.2686-4-59-000 MMBCO0251 04-33.5379-111.2962-4-59-000 4MBC00351 04-33.5255-111.3189-4-59-000 MMBCC0451 04-33.5408-111.3270-4-59-000 MMBC005S1 04-33. MMBC0075.1 D4MMBCOOBS: $04-33$. MMBC00951 04-33.5450-111.4480-4-59-000 MMBCOIOS1 04-33.5247-111.4529-4-59-000 MMBCOIISI 04-33.507?-111.4643-4-59-000 MMBCDI2S1 $04-33.6661-111.4732-4-59-000$ 4MBC01351 $04-33.6333-111.4685-4$ 59-000 MMBC01451 04-33.6233-111.4484-4-59-000 MMBC015S1 04-33.6184-111.4147-4-59-000

CONCENTRATIONS REPORTED IN WEIGHT PARTS PER MILLION (PPM).

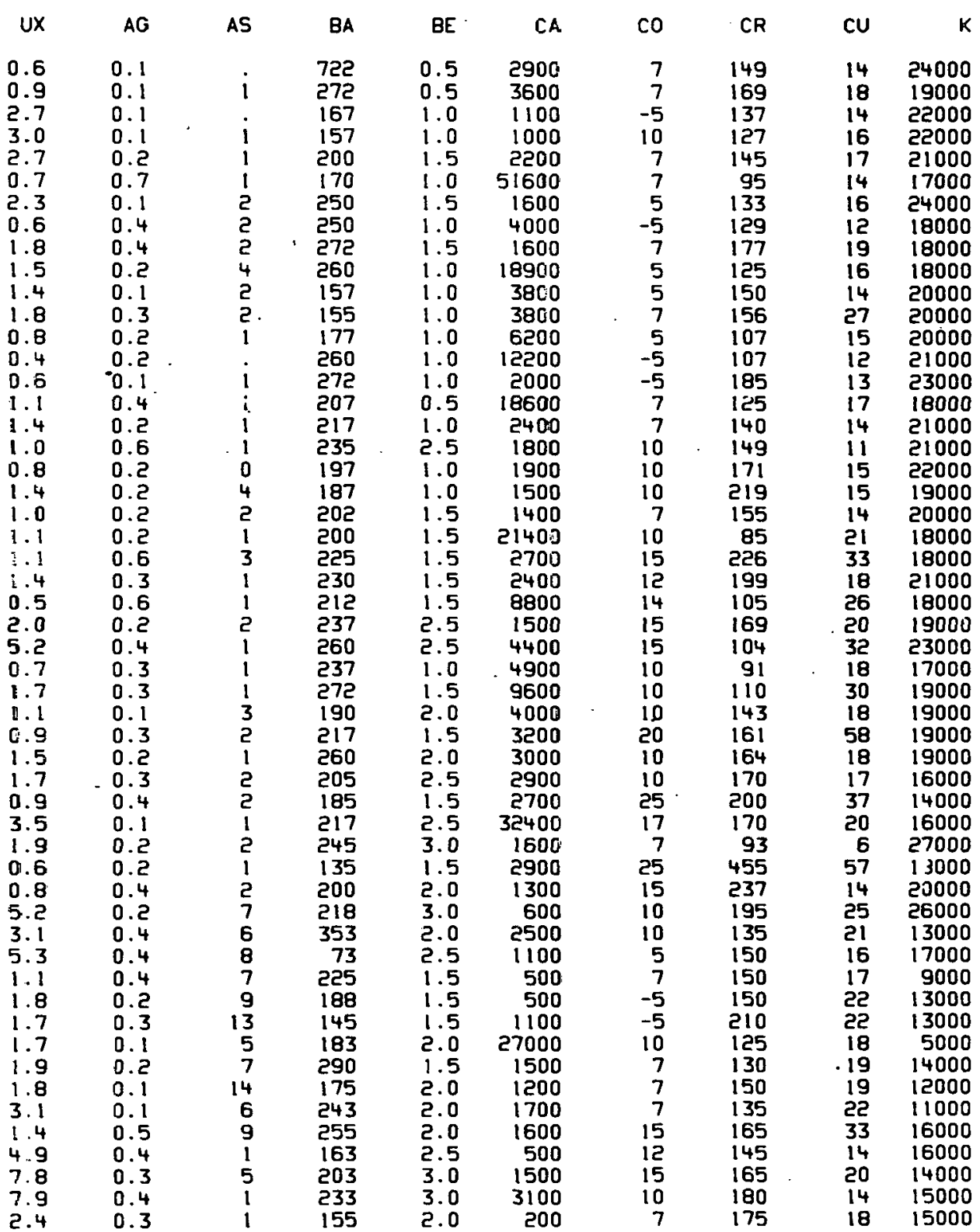


$\cdots+\cdots$

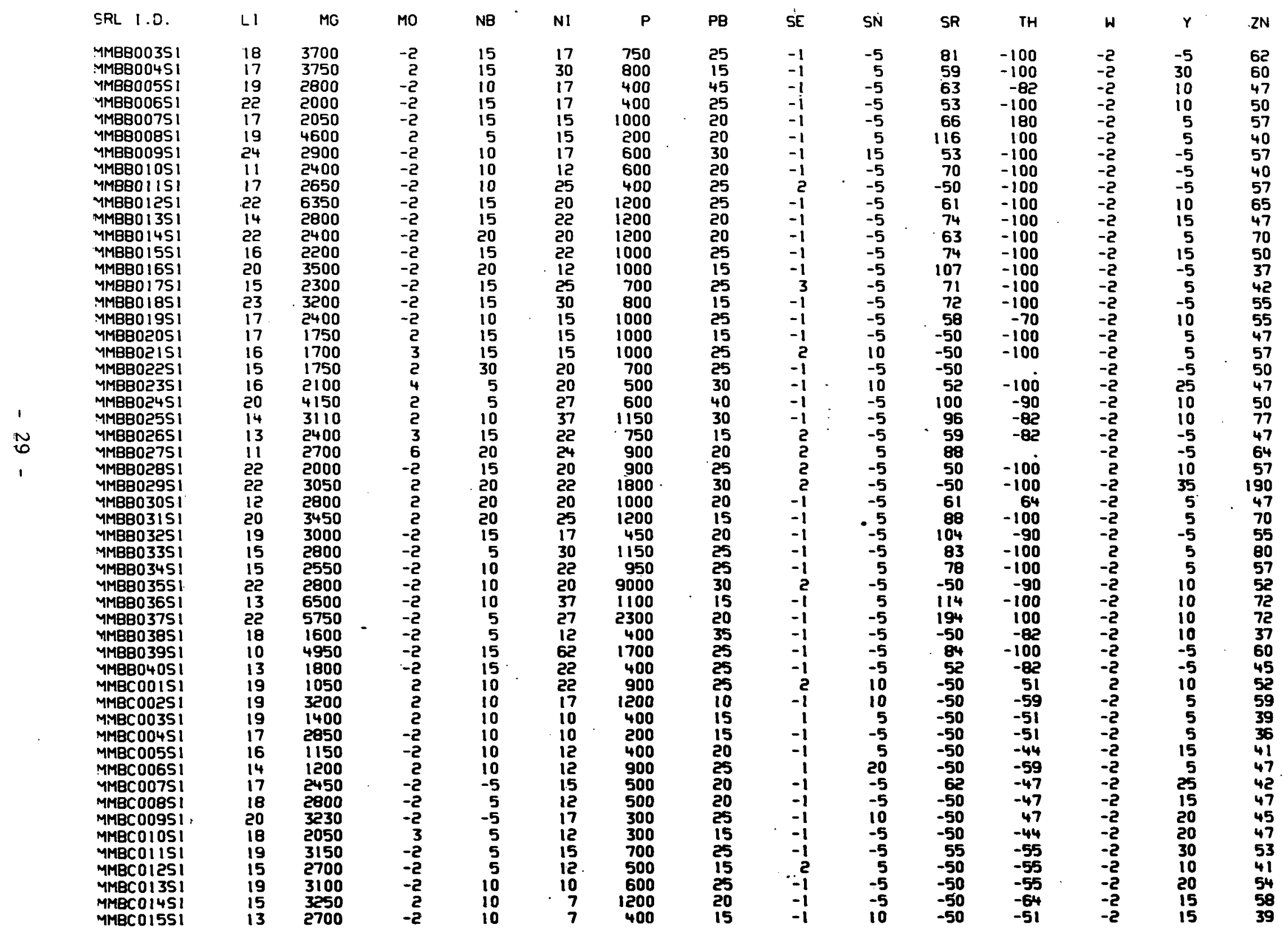

CONCENTRATIONS REPORTEO IN WEIGHT PARTS PER MILLION (PPM). 
$\ldots+4$

SRL I.D. DOE I.D.

MMBC01651 $04-33.6031-111.4231-4-59-000$ MP.01751 04-33.5952-111.4774-4-59-000 MMBCDI851 $04-33.5952-111.4774-4-59-000$ MMCOIBS1 04-33.6232-111.3120-4-59-000 MMBCO21S1 04-33.6278-111.3442-4-59-000 MMBC02251 04-33.7198-1111.2601-4-59-000 MMBC023S1 $04-33.7280-111.2897-4-59-000$ YMBC 02451 $04-33.7138-111.3060-4-59-000$ YMBC025S1 $04-33.7137-111.2754-4-59-000$ YMBC02651 04-33.7463-111.3690-4-59-000 4MBC02751 04-33.7274-111.3546-4-59-000 4MBC029S1 $04-33.7495-111.4957-4-59-000$ 4MBCO30S1 $04-33.5917-111.4368-4-59-000$ 4MBC03151 04-33.7132-111.3918-4-59-000 4MCO3151 04-33. $132-111.3918-4-59-000$ 4MC0351 04 $0433.7257-111.4032-4-59-000$ MMC 03351 04-33.7283-111.4355-4-59-000 MMBC03451 04-33.6833-111.4558-4-59-000 $4 M B C 03551$ 04-33.7094-111.3685-4-59-000 MMBC03651 04-33.7128-111.3349-4-59-000 MMBC037S1 04-33.6829-111.3282-4-59-000 MMBC03851 04-33.6711-111.3322-4-59-000 MMBC03951 04-33.6989-111.3256-4-59-000 MMBCO40S1 04-33.7153-111..4554-4-59-000 MMBDODISI 04-33.5837-111.0172-4-59-000 YMBDO02SI 04-33.5911-111.0617-4-59-000 YMBD00351 $04-33.6492-111.0476-4-59-000$ MMBD0045! 04-33.6466-111.1116-4-59-000 YMBD005S1 04-33.6660-111.0906-4-59-000 YMBD00651 $04-33.6671-111.1286-4-59-000$ MMBD00751 04-33.6245-111. 0597-4-59-000 4MBDO08S1 04-33.6246-111. 0034-4-59-000 MMBD009S1 04-33.6445-111. 0052-4-59-000 MMBD01051 04-33.7027-111.0275-4-59-000 MMBDOIIS1 04-33.6752-111.0358-4-59-000 MMBDIS1 04-33.6752-111.0358-4-59-000 MMBDOI351 04-33.6789-111.0592-4-59-000 MMBD 351 04-33.6789-111.0592-4-59-000 MMBDO 451 04-33.7099-111.0046-4-59-000 MMBDDI5S1 04-33.7386-111.0718-4-59-000 MMBDDI6S1 04-33.7308-111.0894-4-59-000 MMBDCI7SI MMBDOI8SI MMBDOISSI MMBDO20SI MMBDOElsi MMBDO225! MMBD0235 MMBD024S YMBD025S MMBD0265 MMBD0275
CONCENTRATIONS REPORTED IN WEIGHT PARTS PER MLLLION (PPM).

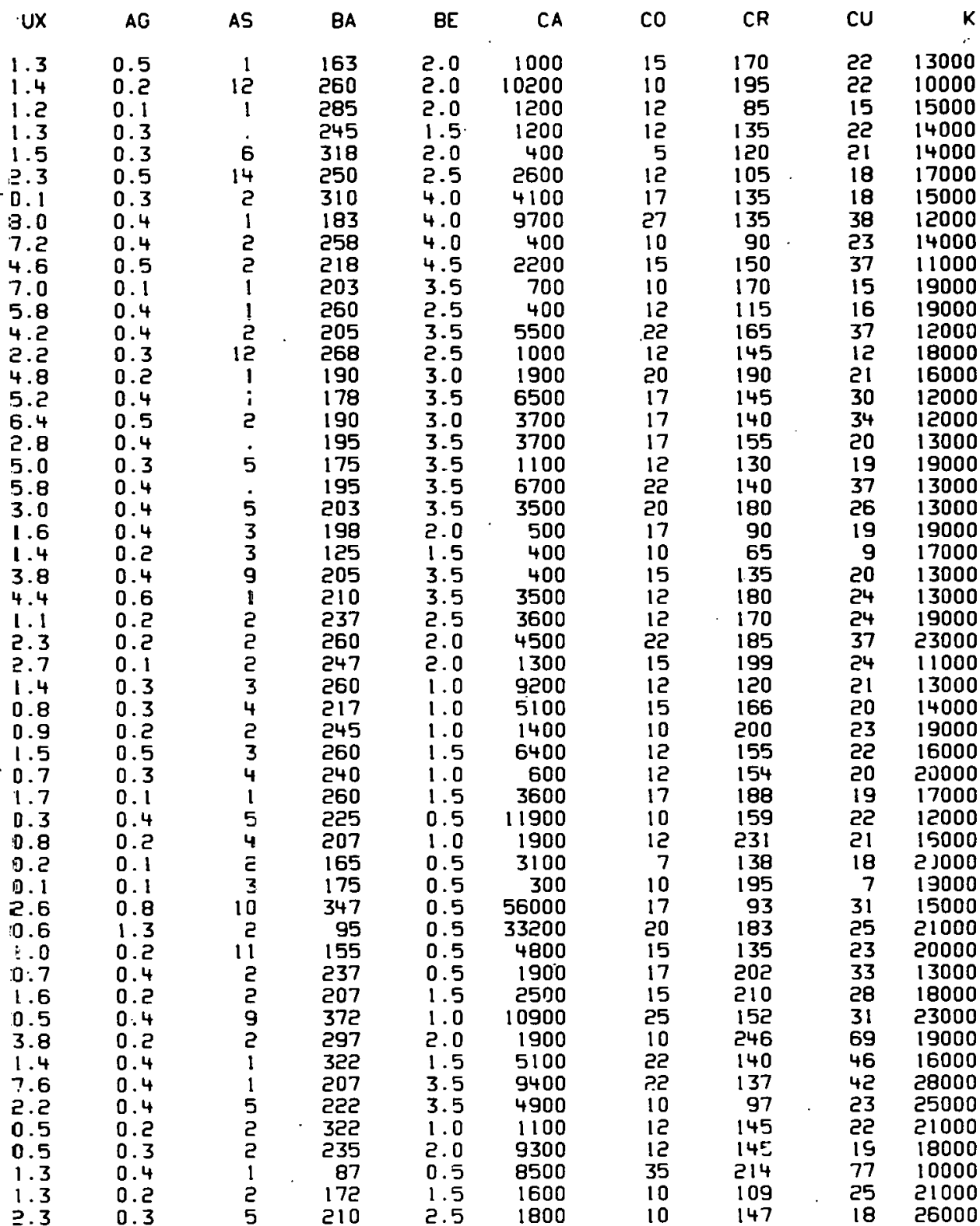


$\ldots+40$

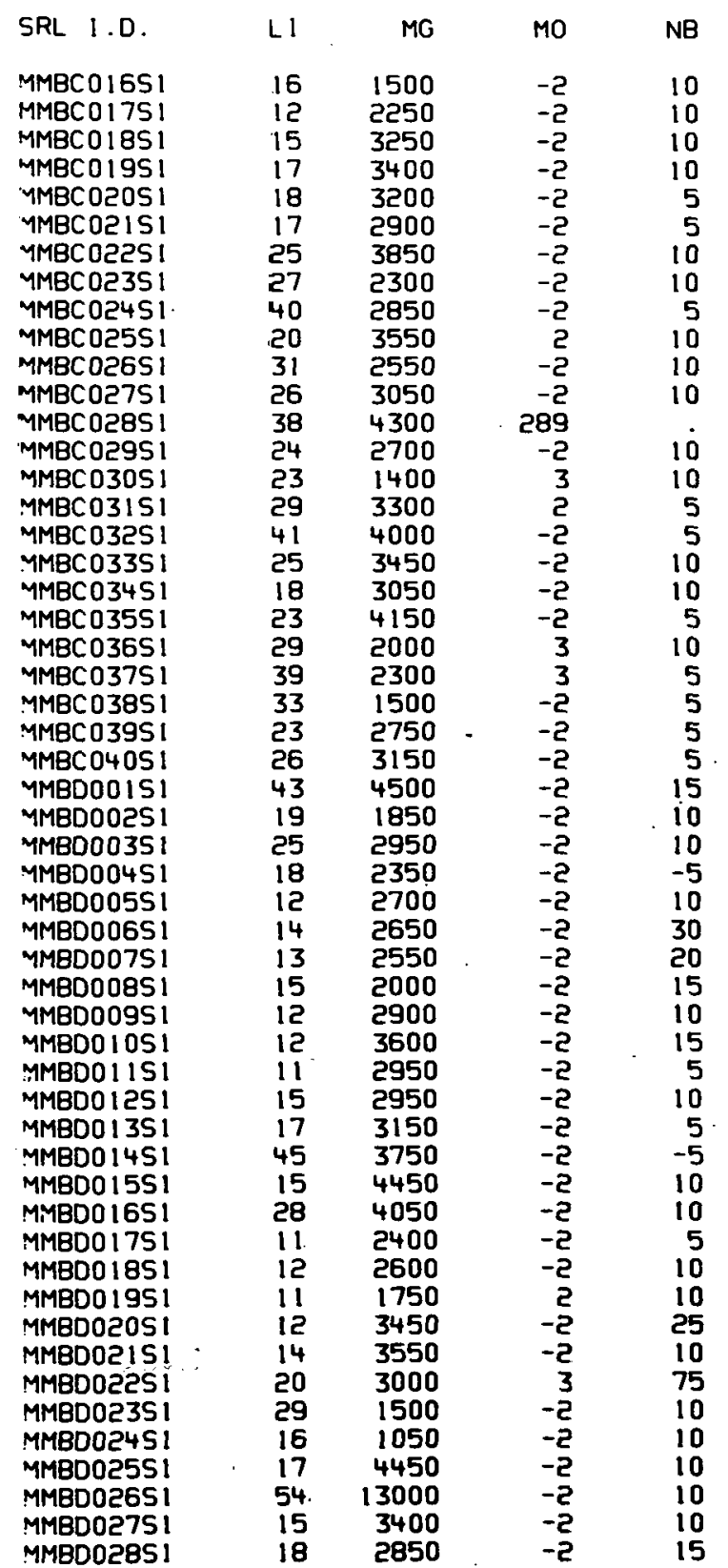

CONCENTRATIONS REPORTED IN WEIGHT PARTS PER MILLION (PPM)

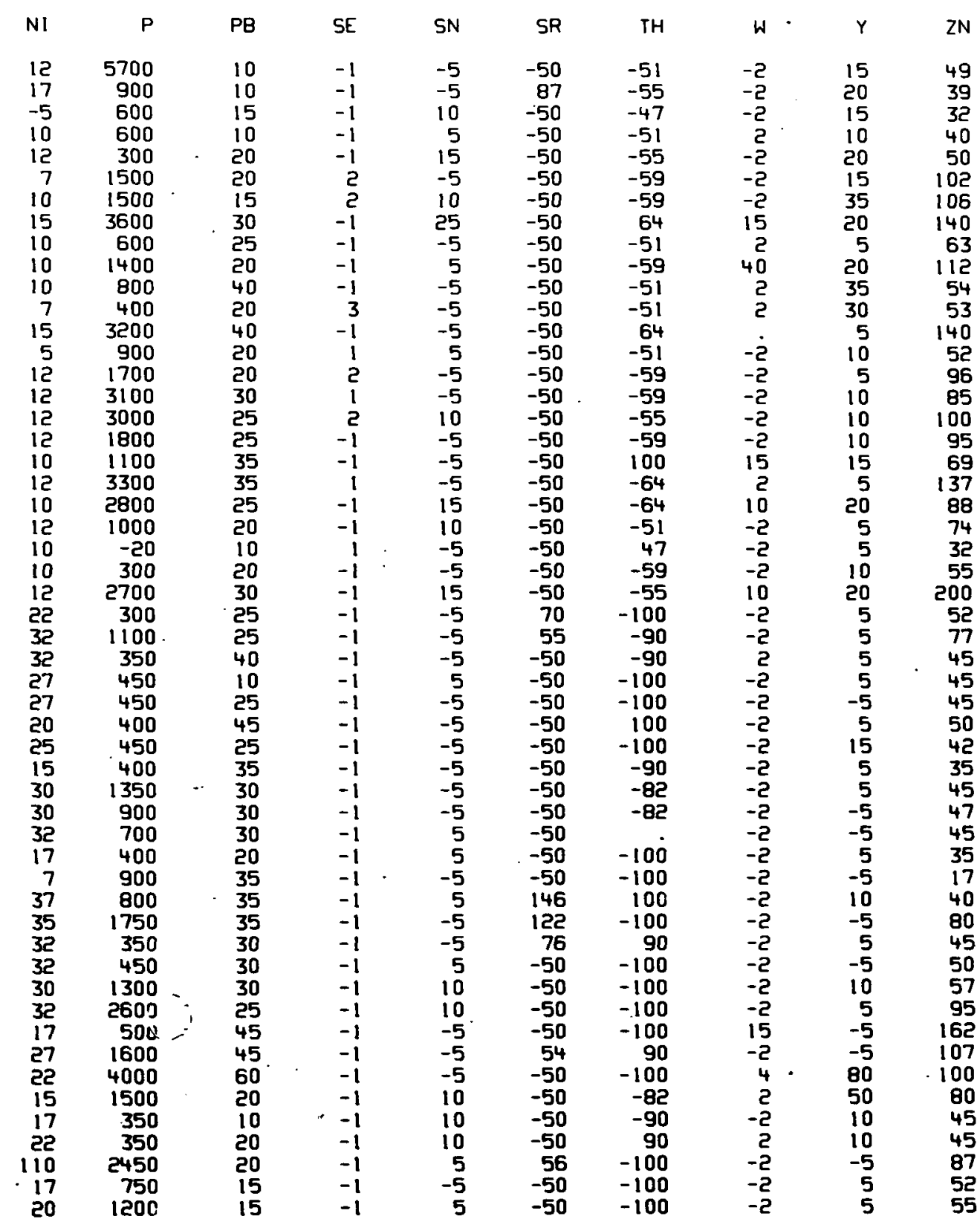


…...

SRL I.D.

DOE I.D

MMBD029S1 04-33.7366-1.11.1275-4-59-000 MMBDO305I MMBD031SI YMBD0325 YMBD0335 MMBD0345 YMBEDOSS

MMBEO035

$M M B E 0045$

MMBE 0055

MMBEODTS

MBEDOOBS

YMBE

MMBEOIIS

MMBEOLISS

MMBEOI 35

MMBED I 45

MMBEDI6S

YMBED 175

YMBEOI8S

MMBEOZOSI

MMBE02IS

YMBE025S

MMBE024S

$4 M B E 025 S$

MMBEO26S

YMBE 275

MMBE 0285

MMBE029S

MMCAODIS

MMCAOOES

MMCAOS

MMCAO04S

MMCAOOSS

MMCACO7S

MMCAD08S

MMCAO09S

MMCAOIOS

MMCAOI1S

MMCAOI2S

MMCAO13S

MMCAO 145

MMCEOD IS

MMCBOD2S

MMCBO035 $04-33.7079-111.1437-4-59-000$ $04-33.5571-111.0716-4-59-000$ (1) 44-33.5413-111.0784-4-59-000 $4-33.5 ! 79-110.8607-4-59-000$ $4-33.5397-110.8375-4-59-000$ 4-33.5435-110.7785-4-59-000 $4-33.5443-110.8061-4-59-000$ 04-33.5594-110.8844-4-59-000 04-33.5791- $110.8171-4-59-000$ 04-33 5907-110.8444-4-59-000 4 04-33.6:13-110.8461-4-59-000 4. 04-33.6094-110 7972-4-59-000 $4-33.6971-110.9799-4-59-000$ 04-33.7208-110.9818-4-59-000 04-33.7208-110.9818-4-59-000 04-33. $7301-110.9546-4-59-000$ 04-33.6977-110.9554-4-59-000 04-33.6739-110.8994-4-59-000 (3).6846- $110.8803-4-59-000$ (1) $4-33.7208-110.8208-4-59-000$ $4-33.5172-110.8851-4-59-000$ $04-33.5244-110.9149-4-59-000$ $04-33.5450-110.9284-4-59-000$ $04-33.5726-110.9512-4-59-000$ 4-33.5999-110.9963-4-59-000 $04-33.6174-110.9289-4-59-000$ $04-33.6194-110.9644-4-59-000$ $04-33.6438-110.9503-4-59-000$ $04-33.6757-110.9734-4-59-000$ $04-33.4549-111.7704-4-59-000$ $04-33.4473-1118135-4-59-000$ $04-33$ 3E 30-111 9816-4-59-000 44-33 3-59-111 9882-4-59-000 $04-33.4805-111.8130-4-59-000$ $04-33.4553-111.9712-4-59-000$ $04-33.4 E 54-111.9508-4-62-000$ 04-33.4E54-111. $9508-4-62-000$ $04-33.4455-111.9363-4-59-000$ $04-33.4 \overline{2} 75-11 \mathrm{r} .9373-4-59-000$ $04-33.4744-111.8359-4-59-000$ $04-33.4 \subseteq 80-111.7896-4-59-000$ $04-33.2504-111.9429-4-59-000$ $04-33.4183-111.5153-459-000$ $04-33.4498-111.5200-4-59-000$ $04-33.4755-111.5160-4-59-000$ $4-33.5690-111.0461-4-59-000$ 04-33.3677-111.9902-4-59-000 $04-33.4515-111.9633-4-59-000$

CONCENTRATIONS REPORTED IN WEIGHT PARTS PER MILLION (PPM).

\begin{tabular}{|c|c|c|c|c|c|c|c|c|c|}
\hline$U x$ & $A G$ & AS & BA & BE & $C A$ & $\mathrm{CO}$ & $C R$ & CU & K \\
\hline 0.3 & 0.2 & 2 & 197 & 1.0 & 2400 & 12 & 253 & 23 & 17000 \\
\hline 0.4 & 0.4 & $\bar{z}$ & 220 & 1.5 & 1900 & 10 & 157 & 18 & 19000 \\
\hline 17.4 & 0.3 & 7 & 167 & 4.0 & 1000 & 10 & 150 & 14 & 21000 \\
\hline 1.0 & 0.3 & 4 & 135 & 1.5 & 5300 & 25 & 245 & 28 & 15000 \\
\hline 1.0 & 0.2 & 2 & 272 & 1.5 & 9000 & 15 & 201 & 22 & 18000 \\
\hline 2.2 & 0.2 & 6 & 245 & 1.5 & 3400 & 10 & 197 & 35 & 18000 \\
\hline 12.0 & 0.2 & 4 & ટ2ર & 2.0 & 3800 & 17 & 192 & 168 & 18000 \\
\hline 0.9 & 0.9 & 6 & 237 & 1.5 & 27000 & 15 & 139 & 60 & 14000 \\
\hline 1.8 & 0.3 & 10 & 422 & 3.5 & 1400 & 17 & 111 & 55 & 40000 \\
\hline 4.9 & 0.1 & 11 & 242 & 5.0 & 5900 & 22 & 197 & 71 & 20000 \\
\hline 5.3 & 0.4 & 12 & ट20 & 4.0 & 10800 & 22 & 163 & 77 & 23000 \\
\hline 3.3 & 0.1 & 6 & 162 & 4.0 & 3100 & 15 & 135 & 50 & 23000 \\
\hline 2.9 & 0.1 & 10. & 230 & 3.5 & 3800 & 12 & 150 & 29 & 26000 \\
\hline 1.4 & 0.4 & 5 & 127 & 1.5 & 46200 & 10 & 130 & 44 & 18000 \\
\hline 1.5 & 0.4 & 4 & 322 & 3.5 & 4700 & 12 & 135 & 43 & 18000 \\
\hline 0.6 & 0.1 & $i$ & 347 & 2.5 & 3900 & 15 & 186 & 34 & 16000 \\
\hline 8.5 & 0.2 & $\overline{2}$ & 397 & 3.5 & 4900 & 15 & 161 & 32 & 23000 \\
\hline 0.4 & 0.6 & $i$ & 372 & 1.0 & $340 \mathrm{C}$ & 17 & 214 & टो & 9000 \\
\hline 1.6 & 0.2 & 1 & 372 & 1.0 & 3200 & 35 & 231 & 33 & 8000 \\
\hline 2.1 & 0.3 & $i$ & 422 & 1.0 & 1900 & 20 & 159 & 24 & 22000 \\
\hline 19.5 & 0.2 & & 260 & 1.0 & 7500 & 37 & 227 & 31 & 10000 \\
\hline 1.7 & 0.7 & 11 & 447 & 3.0 & 3200 & 15 & 178 & 29 & 15000 \\
\hline 1.7 & 0.2 & 4 & 572 & 2.0 & 32900 & 15 & 114 & 21 & 18000 \\
\hline 0.8 & 0.1 & 7 & 322 & 1.0 & 30200 & 20 & 196 & 27 & 14000 \\
\hline 18.0 & 0.3 & 11 & 297 & 5.0 & 8000 & 17 & 139 & 16 & 19000 \\
\hline 1.6 & 0.1 & 3 & 597 & 1.0 & 23300 & 32 & 324 & 24 & 10000 \\
\hline 0.1 & 0.5 & 20 & 397 & 2.0 & 6200 & 50 & 187 & 137 & 13000 \\
\hline 14.2 & 0.3 & 4 & 297 & 4.5 & 4100 & 17 & 140 & 23 & 22000 \\
\hline 52.3 & 0.4 & 10 & 372 & 5.5 & 1400 & 17 & 110 & 82 & 22000 \\
\hline 4.6 & 0.2 & 10 & 272 & 4.0 & 400 & io & 176 & 19 & 21000 \\
\hline 0.7 & 0.1 & 4 & 572 & 1.5 & 10000 & 20 & 167 & 30 & 10000 \\
\hline 0.6 & 0.3 & 6 & 372 & 2.0 & 17900 & 12 & 203 & 28 & 14000 \\
\hline 1.5 & 0.4 & 7 & 647 & 0.5 & 58700 & 15 & 176 & 16 & 16000 \\
\hline 0.7 & 0.1 & 8 & 400 & 1.5 & 3700 & 17 & 194 & 34 & 12000 \\
\hline 0.4 & 0.3 & 5 & 372 & 2.0 & 8900 & 17 & 224 & 20 & 14000 \\
\hline 0.9 & 0.1 & 3 & 372 & 1.5 & 9300 & 17 & 151 & ट2 & 13000 \\
\hline 0.7 & 0.1 & 8 & 397 & 1.0 & રાટ00 & 12 & 148 & 19 & $1 j 000$ \\
\hline 2.1 & 0.3 & 9 & 447 & 2.0 & 13800 & 22 & 190 & 36 & 14000 \\
\hline 0.6 & 0.3 & 2 & 447 & 1.0 & 7500 & 12 & 127 & 18 & 10000 \\
\hline 0.8 & 0.3 & $i$ & 165 & 1.0 & 3000 & 12 & 124 & र2 & 13000 \\
\hline 0.7 & 0.2 & 6 & 245 & 1.0 & 2300 & 10 & 154 & 14 & 8000 \\
\hline 2.8 & 0.1 & 9 & 372 & 2.0 & 13800 & 22 & 185 & 41 & 13000 \\
\hline & & & 372 & 1.0 & & & & & 11000 \\
\hline 0.9 & 0.2 & 4 & 285 & 1.0 & 10300 & 12 & 151 & 15 & 14000 \\
\hline 1.0 & 0.4 & 6 & 422 & 2.0 & 7600 & 15 & 146 & 18 & 14000 \\
\hline 0.8 & 0.2 & 6 & 372 & 1.5 & 9600 & 15 & 157 & 28 & 16000 \\
\hline 0.7 & 0.4 & 6 & 347 & 1.0 & 24600 & 15 & 148 & 15 & 14000 \\
\hline & • & . & & & & • & & • & \\
\hline & & & 307 & & & & & & \\
\hline 0.6 & 0.3 & 2 & 397 & 1.5 & 2200 & 12 & 117 & 14 & 11000 \\
\hline 0.9 & 0.1 & 4 & 422 & - 1.5 & 1100 & 12 & 146 & 16 & 18000 \\
\hline $\begin{array}{l}2.7 \\
0.7\end{array}$ & $\begin{array}{l}0.1 \\
0.2\end{array}$ & $\begin{array}{l}5 \\
4\end{array}$ & $\begin{array}{l}472 \\
332\end{array}$ & $\begin{array}{l}2.0 \\
2.0\end{array}$ & $\begin{array}{l}1700 \\
4500\end{array}$ & $\begin{array}{l}12 \\
20\end{array}$ & $\begin{array}{l}171 \\
251\end{array}$ & $\begin{array}{l}22 \\
71\end{array}$ & $\begin{array}{l}23000 \\
15000\end{array}$ \\
\hline
\end{tabular}


$+\ldots+\infty$

SRL I.D

MMBD029SI MMBD030S MMBD031S MMBD032S MMBD0335 MMBD034S YMBE 0015 YMBE 0025 YMBE003S

YMBE004S

MMBE005SI

MMBE006S1

MMBE 00751

MMBE0085 I

MMBE009S

MMBEOIOSI

MMBE OIIS

MMBEOIDS

MMBE 01351

MMBED I5S

MMBEO I6S

MMBEOI 75

MMBEO1851

MMBEOISSI

MMBEOZOS

MMBE 02 IS

MMBEO2ZSI

MMBE 2451

MMBE025S

MMBE 026S

MMBE OE7S

MMBE0Z8SI

MMBE029S

MMCAODESI

MMCA0035I

MMCAO045

MMCA0055

MMCA006SI

MMCA0075

MMCAOOBS

MMCAO 1051

MMCAO I ISI

MMCAOIZSI

MMCAO I 3S1

MMCAO 1451

MMCAO 15SI

MMCBODISI

MMCB0035

CONCENTRATIONS REPORTED IN WEIGHT PARTS PER MILLION (PPM).

$\begin{array}{rrrr}L 1 & M G & M O & N B \\ 14 & 3900 & -2 & 10 \\ 15 & 3250 & -2 & 19 \\ 21 & 2500 & -2 & 20 \\ 34 & 5000 & 2 & 15 \\ 14 & 1350 & -2 & 10 \\ 18 & 3650 & 2 & 15 \\ 23 & 3650 & 2 & 15 \\ 23 & 2900 & -2 & 15 \\ 18 & 2950 & -2 & 25 \\ 29 & 4150 & 3 & 40 \\ 39 & 4350 & -2 & 45 \\ 32 & 3000 & 2 & 30 \\ 20 & 2800 & -2 & 40 \\ 18 & 2150 & -2 & 10 \\ 18 & 1100 & -2 & 10 \\ 16 & 1400 & 3 & 5 \\ 19 & 900 & -2 & 15 \\ 36 & 900 & -2 & 5 \\ 8 & 2400 & -2 & 5 \\ 5 & 1300 & -2 & 10 \\ 7 & 5000 & -2 & 10 \\ 6 & 1100 & -2 & 10 \\ 6 & 1700 & -2 & 5 \\ 10 & 4300 & -2 & 5 \\ 22 & 700 & -2 & 40 \\ 17 & 2900 & -2 & 10 \\ 15 & 2300 & -2 & 5 \\ 28 & 1100 & -2 & 10 \\ 17 & 500 & -2 & 40 \\ 20 & 300 & -2 & 15 \\ 14 & 1600 & -2 & 10 \\ 51 & 1600 & -2 & 15 \\ 10 & 1500 & -2 & 10 \\ 15 & 1400 & -2 & 10 \\ 8 & 1800 & -2 & 10 \\ 7 & 1400 & -2 & 20 \\ 5 & 1600 & -2 & 25 \\ 7 & 1100 & -2 & 10 \\ 11 & 1100 & -2 & 15 \\ 7 & 700 & -2 & 15 \\ 6 & 2800 & -2 & 15 \\ 9 & 1500 & -2 & 35 \\ -5 & 2400 & -2 & 10 \\ -5 & 1200 & -2 & -2 \\ 5 & 800 & -2 & 25 \\ 5 & 1800 & -2 & 40 \\ -5 & 1600 & -2 & 10 \\ \cdot & -2 & - & \\ 5 & 800 & -2 & 10 \\ \mathbf{6} & 1000 & -2 & 10 \\ 5 & 1100 & -2 & 25 \\ 5 & 1300 & -2 & 15 \\ & & & \end{array}$

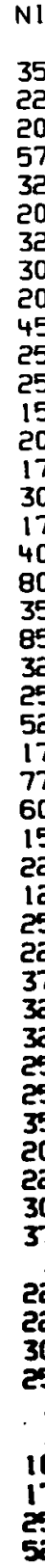

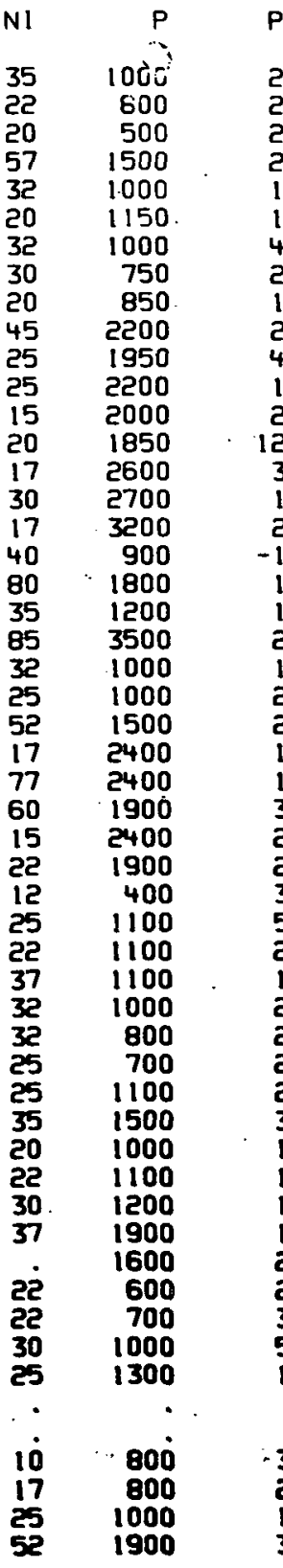

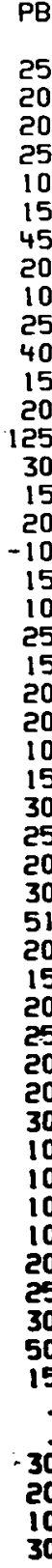

$S E$
-1
-1
-1
-1
-1
-1
-1
-1
-1
-1
-1
-1
-1
-1
-1
-1
-1
-1
-1
-1
-1
-1
-1
-1
-1
-1
-1
-1
-1
-1
-1
-1
-1
-1
-1
-1
-1
-1
-1
-1
-1
-1
-1
-1
-1
-1
-1
-1
-1
-1
-1
-1
-1

SN

SR TH

W $\quad$ Y

ZN

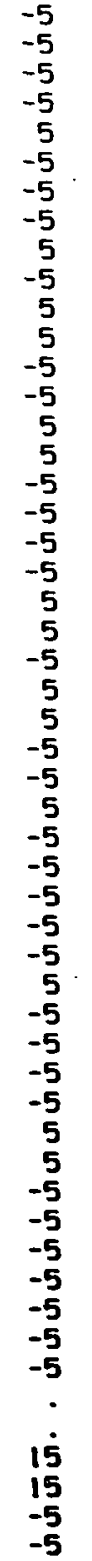

-50
-50
-50
-50
-50
-50
-50
53
-50
-50
-50
-50
-50
137
-50
-50
-50
-50
-50
-50
50
-50
-50
140
-50
60
-50
-50
-50
-50
-50
-50
66
-50
-50
-50
-50
-50
-50
-50
80
-50
-50
-50
-50
-50
86
-50
-50
-50
-50
-50

100
-100

$-100$

$-100$

100
-90

$-100$

$-100$

$-82$

-100
-100

$-100$

$-100$

$-100$

$-100$

-100
-100

-100
-100

100

$-100$

$-100$

$-100$

-100
-100
-100

$-100$

$-100$

100

100
-100
90

$-90$

-100
-100

-100
-90

-90
-100

$-100$

$-90$

100
-100

$-100$

$-100$

$-100$

$-100$

$-100$

$-100$

$-100$

-2
-2
-2
-2
-2
-2
-2
-2
-2
-2
-2
4
-2
3
-2
20
-2
-2
-2
-2
-2
-2
-2
-2
-2
10
-2
-2
-2
37
15
-2
-2
-2
-2
2
-2
-2
2
-2
-2
-2
-2
-2
-2
-2
-2
-2
-2
-2
-2
-2
-2
-2
-2

$\begin{array}{rr}10 & 4 \\ -5 & 3 \\ -5 & 5 \\ 5 & 70 \\ 5 & 4 \\ 5 & 57 \\ 5 & 167 \\ -5 & \\ 15 & \\ 45 & \\ 20 & 155 \\ 20 & \\ 20 & \\ 5 & 17 \\ 45 & \end{array}$

47
37
52
70
42
57
67
62
52
82
55
92
75
72

25

20
5

$-100$

$-100$

-100
100

-2
-2
-2
-2
-2

5
10
20
-5 
SRL 1.0

DOE I.D.

MMCB00451 04-33.4471-111.5623-4-59-000 YMC 3005Si $04-33.4790-111.5726-4-61-500$ 4MCBODSS1 $04-33.4481-111.6045-4-59-000$ 作 4MCB00851 04-33.4085-11. $5624-4-59-000$ $04-33.3784-111.5630-4-59-000$ MMCBDIOSI $04-33.3402-111.5630-4-59-000$ MMCBOIISI 04-33.3109-111.5629-4-59-000 YMCBOIวS1 04-33.2710-111.5622-4-59-000 4MCB01351 04-33.2638-111.5278-4-59-000 YMCBO145 $04-33.2625-111.6070-4-59-000$ 4MCBD15S1 04-33. $2051-111.6057-4-59-000$ YMCBDI5S $04-33.3061-111.6057-4-59-000$ 4MCBO1651 04-33. $2920-111.6347-4-59-000$ YMCBOIBSI 04-33.4077-111.6317-4-59-000 . MMCBOISS! 04-33.3410-111.5182-4-59-000 MMCBOEOS1 04-33.2957-111.5279-4-59-000 MMCBOCISI 04-33.3353-111.6440-4-59-000 4MCBOZ2S1 04-33.3787-111.6447-4-59-000 MMCBO2351 04-33.3749-111.6007-4-59-000 MMCB024S1 04-33.3426-111.6033-4-59-000 MMCB025S1 04-33.2713-111.6587-4-59-000 MMCB02651 04-33.2659-111. 6853-4-59-000 YMCB02751 04-33.2700-111.7373-4-59-000 MMCBO28S1 04-33.3054-111.7223-4-59-000 YMCBOZ9S1 04-33.3046-111.6882-4-59-000 YMCB03051 04-33.3498-111.6806-4-59-000 151 $04-33.3222-111.7405-4-59-000$ 4MCB032S1 04-33.4088-11!.6841-4-59-000 YMCBO3351 04-33.3798-11:.6865-4-59-000 YMCBO351 04-33.3644-11:.7166-4-59-000 YMCBO355! 04-33.4102 $11 ! .7104-4-59-000$ MMCB03651 04-33.4487-111.7347-4-59-000 MMCB03751 04-33.4438-111.6362-4-59-000 MMCB03851 04-33.4783-111.6487-4-59-000 YMCB03951 04-33.4736-111.6133-4-59-000 YMCBO40S1 04-33.4805-111.6841-4-59-000 MMCBO4ISI 04-33.4453-111.6841-4-59-000 MMCBO4टS1 $04-33.4815-111.7185-4-59-000$ YMCCOOISI $04-33.3730-111.4848-4-59-000$ YMCCOO2S1 $04-33.3431-111.4708-4-59-000$ 4MCCC0351 04-33.4125-111.4102-4-59-000 MMCC $00451 \quad 04-33.4075-111.4435-4-59-000$ MMCC 005S1 $04-33.3358-111.4403-4-59-000$ MMCCOD6S1 $04-33.3069-111.4763-4-59-000$ MMCCOD751 $04-33.3109-111.4459-4-59-000$ MMCC 008SI $04-33.3362-111.3938-4-56-000$ MMCCO09S1 04-33.2998-111.3943-4-59-000 MMCCO1051 $04-33.2748-111.3925-4-59-000$ $04-33.2685-111.4404-4-59-000$ MMCCOIZSI 04-33.2717-111.4931-4 59-000 MMCCOI3S1 04-33.2671-111.4931-4 59-000 MMCCOI3S1. $04-33.267 !-111.3510-4-59-000$
CONCENTRATIONS REPORTED IN WEISHT PARTS PER MILLION (PPM).

$\begin{array}{ll}\text { UX } & \text { AG } \\ 1.8 & 0.4 \\ 1.0 & 0.1 \\ 1.9 & 0.2 \\ 2.4 & 0.5 \\ 0.9 & 0.0 \\ 0.9 & 0.3 \\ 0.6 & 0.1 \\ 1.1 & 0.4 \\ 1.0 & 0.3 \\ 0.8 & 0.2 \\ 0.6 & 0.1 \\ 0.9 & 0.1 \\ 0.7 & 0.3 \\ 2.6 & 0.4 \\ 1.5 . & 0.3 \\ 1.0 & 0.4 \\ 0.8 & 0.4 \\ 0.6 & 0.2 \\ 0.7 & 0.6 \\ 1.6 & 0.1 \\ 0.6 & 0.6 \\ 0.8 & 0.2 \\ 0.6 & 0.3 \\ 1.6 & 0.3 \\ .1 .0 & 0.4 \\ 1.0 & 0.4 \\ 3.2 & 0.4 \\ 0 . E & 0.2 \\ 1.0 & 0.5 \\ 1 . \bar{C} & 0.1 \\ 1.5 & 0.1 \\ 1.0 & 0.4 \\ 1.6 & 0.4 \\ 0.7 & 0.5 \\ 6.2 & 0.1 \\ 3.8 & 0.2 \\ 5.4 & 0.7 \\ 2.0 & 0.7 \\ 1.2 & 0.5 \\ 0.9 & 0.6 \\ 0.7 & 0.3 \\ 1.0 & 0.1 \\ 0.5 & 0.1 \\ 0.8 & 0.2 \\ 1.0 & 0.4 \\ 2.6 & 0.6 \\ 1.6 & 0.5 \\ 0.8 & 0.1 \\ 0.5 & 0.3 \\ 0.8 & 0.4 \\ 0.9 & 0.1 \\ 0.6 & 0.3 \\ 0.6 & 0.2 \\ & \end{array}$

A

$\begin{array}{rrrrr}\text { BA } & \text { BE } & \text { CA } & \text { CD } & \text { CR } \\ 297 & 2.0 & 1300 & 12 & 152 \\ 297 & 2.0 & 800 & 7 & 95 \\ 190 & 1.5 & 1300 & 7 & \\ 272 & 2.0 & 1100 & 7 & 76 \\ 250 & 1.5 & 5000 & 12 & 53 \\ 347 & 1.5 & 1500 & 10 & 66 \\ 192 & 1.5 & 72 & 52 \\ 322 & 2.0 & 1700 & 7 & 69 \\ 297 & 2.0 & 1200 & 15 & 69 \\ 322 & 2.0 & 1200 & 7 & 50 \\ 322 & 2.0 & 1400 & 10 & 87 \\ 372 & 1.5 & 3700 & 12 & 88 \\ 245 & 2.0 & 1400 & 7 & 49 \\ 297 & 2.0 & 5400 & 15 & 179 \\ 260 & 2.0 & 1700 & 10 & 128 \\ 322 & 2.0 & 1400 & 10 & 144 \\ 297 & 2.0 & 800 & 10 & 166 \\ 297 & 1.5 & 5300 & 12 & 145 \\ 297 & 1.5 & 2000 & 10 & 128 \\ 260 & 2.0 & 1900 & 10 & 192 \\ 260 & 2.0 & 900 & 7 & 77 \\ 260 & 2.0 & 1500 & 7 & 86 \\ 297 & 2.0 & 2500 & 10 & 119 \\ 272 & 1.5 & 4400 & 12 & 182 \\ 297 & 2.0 & 3800 & 10 & 160 \\ 297 & 2.0 & & 150 \\ 272 & 1.5 & 4600 & 15 & 137 \\ 260 & 2.0 & 3600 & 12 & 126 \\ 347 & 2.0 & 1800 & 10 & 150 \\ 202 & 1.5 & 2400 & 12 & 142 \\ 272 & 2.0 & 1300 & 10 & 125 \\ 245 & 2.0 & 7500 & 7 & 139 \\ 272 & 1.5 & 1200 & 15 & 136 \\ 260 & 2.0 & 1000 & 10 & 145 \\ 167 & 2.0 & 11000 & 15 & 211 \\ 230 & 2.0 & 900 & 7 & 194 \\ 260 & 2.0 & 3500 & 7 & 178 \\ 212 & 1.5 & 6300 & 10 & 182 \\ 260 & 1.5 & 26400 & 10 & 181 \\ 260 & 2.0 & 3600 & 12 & 186 \\ 272 & 2.0 & 900 & 10 & 143 \\ 260 & 2.0 & 2000 & 10 & 162 \\ 248 & 2.0 & 1300 & 10 & 117 \\ 373 & 1.5 & 18100 & 10 & 110 \\ 200 & 1.5 & 1500 & 12 & 193 \\ 273 & 1.5 & 1500 & 7 & 137 \\ 248 & 1.5 & 1200 & 7 & 159 \\ 260 & 1.5 & 1500 & 10 & 146 \\ 245 & 1.5 & 600 & 7 & 137 \\ 240 & 1.5 & 2100 & 10 & 145 \\ 153 & 1.5 & 700 & 7 & 141 \\ 198 & 1.5 & 1000 & 10 & 144 \\ 230 & 2.0 & 5600 & 15 & 132 \\ 243 & 1.5 & 1300 & 10 & 143\end{array}$

$\begin{array}{lr}\text { CU } & \text { K } \\ 17 & 10000 \\ 25 & 19000 \\ 22 & 15000 \\ 22 & 18000 \\ 19 & 17000 \\ 19 & 25000 \\ 16 & 14000 \\ 21 & 21000 \\ 19 & 18000 \\ 20 & 24000 \\ 25 & 18000 \\ 18 & 22000 \\ 24 & 19000 \\ 15 & 18000 \\ 18 & 20000 \\ 20 & 18000 \\ 19 & 18000 \\ 17 & 17000 \\ 19 & 18000 \\ 14 & 22000 \\ 21 & 22000 \\ 17 & 19000 \\ 41 & 14000 \\ 44 & 17000 \\ 13 & 15000 \\ 33 & 16000 \\ 23 & 21000 \\ 17 & 18000 \\ 44 & 22000 \\ 13 & 16000 \\ 17 & 18000 \\ 22 & 19000 \\ 17 & 14000 \\ 19 & 17000 \\ 14 & 18000 \\ 15 & 19000 \\ 15 & 13000 \\ 20 & 21000 \\ 19 & 18000 \\ 21 & 29000 \\ 18 & 17000 \\ 28 & 23000 \\ 15 & 16000 \\ 21 & 19000 \\ 19 & 21000 \\ 21 & 17000 \\ 28 & 13000 \\ 38 & 21000 \\ 30 & 20000 \\ 21 & 12000 \\ 18 & 22000 \\ 22 & 17000 \\ 37 & 13000 \\ & \end{array}$




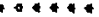

CONCENTRATIONS REPORTED IN WEIGHT PARTS PER MILLION (PPM).

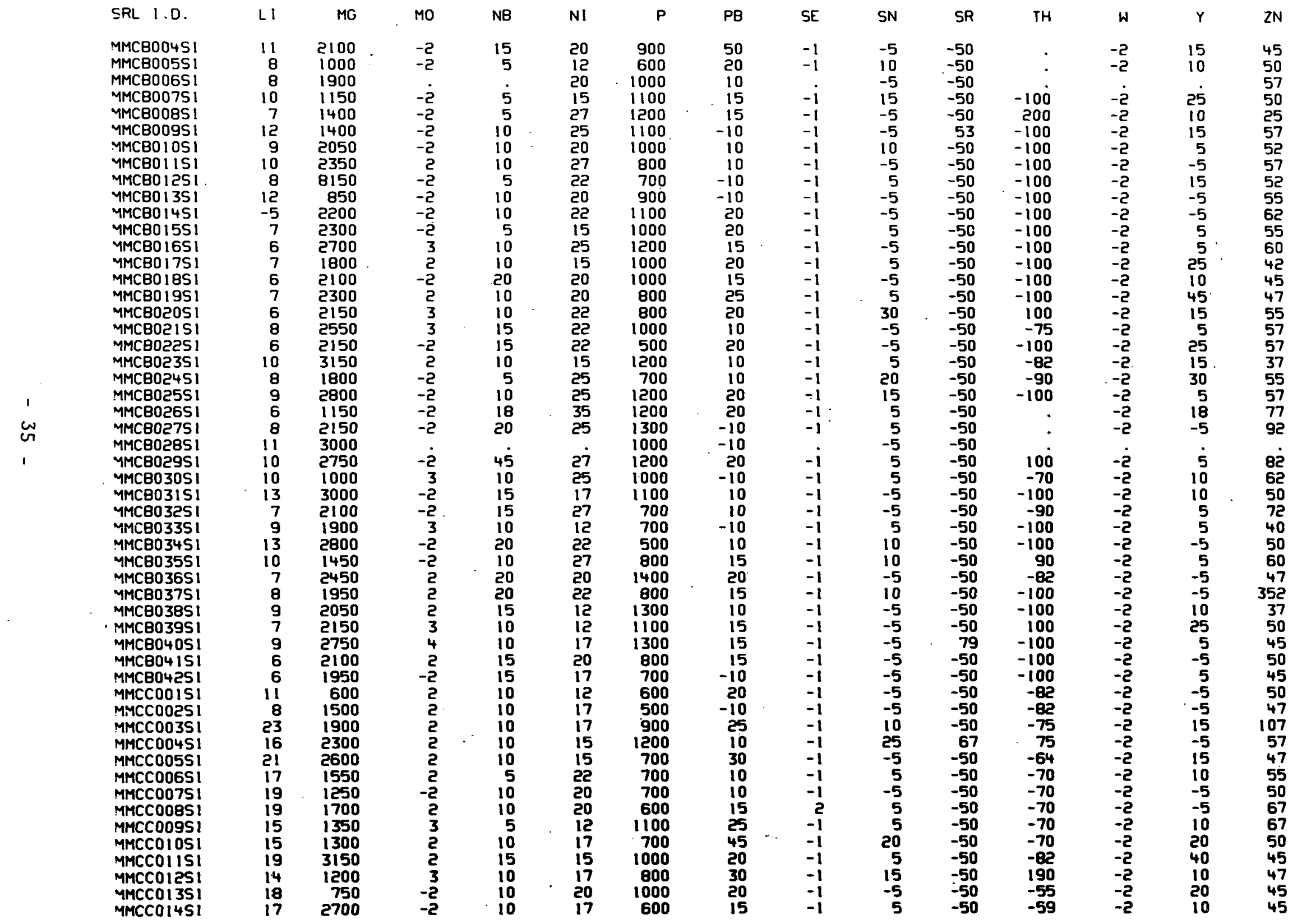


$0 .+0$.

DOE $\mathrm{i} . \mathrm{\Omega}$.

MMCCO1551 04-33.2632-111.2703-4-59-000 MiMC 01651 04-33.3005-111.2763-4-59-000 $4 M C C 01751$ YMCCDITS1 04-33.2966-111.3146-4-59-000 YMCCDI951 $04-33.3277-111.3254-4-59-000$ MMCC02051 $04-33.3516-111.3431-4-59-000$ MMCCODIS1 $04-33.4488-111.4847-4-59-000$ MMCCO2151 $04-33.4488-111.4847-4-59-000$ 4MCC $0235104-33.4782-111.4417-4-59-000$ 4MCCO2451 $04-33.4782-111.4417-4-59-000$ 4MCCOLS1 04-33.4154-111.4850-4-59-000 4MCCO25S1 $04-33.3785-111.4356-4-59-000$ 4MCC02651 $04-33.3644-111.3828-4-59-000$ MMCC02751 $04-33.3780-111.3639-4-59-000$ MMCCO2851 $04-33.3960-111.3485-4-59-000$ YMCCO29S1 $04-33.3934-111.3203-4-59-000$ YMCCO30S1 04-33.3333-111.2804-4-59-000 YMCC03151 04-33.3675-111.2712-4-59-000 MMCC 032S1 04-33.4586-111.4505-4-59-000 YMCC03351 04-33.4534-111.4044-4-59-000 YMCC034S1 $04-33.4585-111.4161-4-59-000$ MMCC035S1 04-33.4921-111.2847-4-59-000 MMCC03651 04-33.4993-111. 2977-4-59-000 4MCD00151 04-33.2824-111.1473-4-59-000 4MCD002S1 04-33.2711-111.1832-4-59-000 YMCD00351 $04-33.2743-111.2213-4-59-000$ MMCO004S1 $04-33.3035-111.2325-4-59-000$ YMCDO05S1 04-33.2987-111.1962-4-59-000 MMCD006S1 $04-33.3213-111.1654-4-59-000$ MMCD00751 04-33.3098-111.1408-4-59-000 4MCDO0851 04-33.3386-111.1346-4-59-000 MMCDOO9SL 04-33.3390-111.2264-4-59-000 MMCDO10S1 04-33.3586-111.2092-4-59-000 MMCDOIISL 04-33.3781-111.1921-4-59-000 MMCDOL LSL $04-33.4194-111.0331-4-59-000$ MMCD01351 $04-33.4257-111.0540-4-59-000$ MMCDO145 $04-33.4366-111.0688-4-59-000$ MMCDO15SE $04-33.4282-111.0865-4-59-000$ MMCDO1651 $04-33.4285-111.0073-4-59-000$ YMCD01751 04-33.4472-111.0102-4-59-000 MMCO018S1 04-33.4674-111.0267-4-59-000 (951 $04-33.4680-111.0250-4-59-000$ 4MCDO2051 04-33.2619-111. 0388-4-59-000 MMCDODIS1 $04-33.3147-111.0354-4-59-000$ 1 $04-33.3401-111.0154-4-59-000$ 4MCDO2351 04-33.3625-111.0031-4-59-000 4MCD02451 04-33.3397-111.0627-4-59-000 MMCDO25SI 04-33.3397-111.0627-4-59-000 MMCDOEST: 04-33.3066-111. MMCDOES: 04-33.3286-111.0948-4-59-000 MMCDOC25: 04-33.3020-111.0593-4-59-000 04-33.2717-111. $1131-4-59-000$ 04 $33.2797-111.0455-459-000$ $\begin{array}{ll}\text { MMCD030SI } & 04-33.3985-111.1961-4-59-000 \\ \text { MMCD031S1 } & 04-33.413 E-111.1897-4-59 \cdot 000\end{array}$

CONCENTTRATIONS REPORTED IN WEIGHT PARTS PER MILLION (PPM).

$\begin{array}{ll}\text { UX } & \text { AG } \\ 0.8 & 0.2 \\ 1.0 & 0.5 \\ 1.1 & 0.4 \\ 1.3 & 0.2 \\ 0.6 & 0.5 \\ 0.7 & 0.2 \\ 0.6 & 0.2 \\ 0.7 & 0.1 \\ 0.7 & 0.2 \\ 0.5 & 0.1 \\ 0.7 & 0.4 \\ 1.0 & 0.2 \\ 0.9 & 0.2 \\ 1.4 & 0.3 \\ 0.8 & 0.3 \\ 0.7 & 0.2 \\ 1.2 & 0.6 \\ 0.7 & 0.3 \\ 0.8 & 0.3 \\ 0.7 & 0.1 \\ 1.2 & 0.1 \\ 0.9 & 0.1 \\ 0.4 & 0.2 \\ 0.8 & 1.1 \\ 1.1 & 0.4 \\ 2.2 & 0.5 \\ 0.7 & 0.2 \\ 1.0 & 0.1 \\ 0.7 & 0.2 \\ 0.6 & 0.4 \\ 0.8 & 1.4 \\ 0.4 & 0.2 \\ 2.0 & 0.5 \\ 0.3 & 0.5 \\ 0.4 & 0.5 \\ 0.3 & 0.6 \\ 1.5 & 0.9 \\ 0.6 & 0.2 \\ 0.9 & 0.2 \\ 0.5 & 0.6 \\ 0.4 & 0.2 \\ 0.8 & 0.2 \\ 0.8 & 0.3 \\ 0.6 & 0.2 \\ 0.5 & 0.4 \\ 1.2 & 0.3 \\ 0.6 & 4.1 \\ 0.4 & 0.8 \\ 0.5 & 0.4 \\ 0.9 & 0.5 \\ 0.6 & 0.5 \\ 7.1 & 1 . E \\ 6.4 & 0.5 \\ & \\ 0.4 & \end{array}$

A5

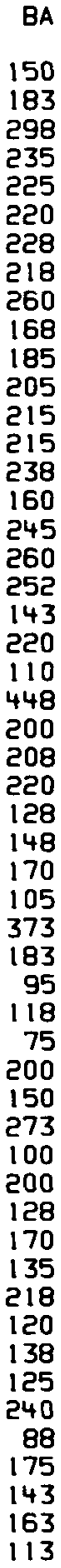

$\begin{array}{rrrr}\text { BE } & \text { CA } & \text { CO } & \text { CR } \\ 1.5 & 900 & 12 & 122 \\ 1.0 & 5100 & 12 & 144 \\ 1.5 & 600 & 7 & 178 \\ 1.5 & 600 & 10 & 184 \\ 1.5 & 600 & 10 & 144 \\ 1.5 & 1200 & 7 & 133 \\ 1.0 & 4900 & 10 & 140 \\ 1.0 & 2400 & 12 & 117 \\ 1.5 & 800 & 12 & 146 \\ 1.5 & 3100 & 10 & 142 \\ 1.5 & 800 & 10 & 157 \\ 1.5 & 800 & 12 & 199 \\ 1.0 & 1100 & 10 & 162 \\ 1.0 & 2300 & 12 & 194 \\ 2.0 & 800 & 12 & 115 \\ 1.5 & 1100 & 10 & 164 \\ 1.0 & 10600 & 15 & 229 \\ 1.0 & 2500 & 10 & 151 \\ 1.0 & 700 & 7 & 129 \\ 1.0 & 3100 & 10 & 109 \\ 2.0 & 1200 & 15 & 135 \\ 2.0 & 1400 & 10 & 121 \\ -0.5 & 2700 & 20 & 187 \\ 1.0 & 50000 & 15 & 127 \\ 1.5 & 800 & 12 & 173 \\ 1.5 & 1100 & 17 & 178 \\ 1.0 & 3300 & 15 & 276 \\ 1.5 & 2200 & 12 & 123 \\ 2.0 & 2200 & 22 & 285 \\ 1.5 & 3600 & 27 & 316 \\ 1.5 & 79500 & 12 & 101 \\ 1.5 & 3600 & 27 & 239 \\ 3.0 & 700 & 12 & 182 \\ 1.5 & 3900 & 17 & 129 \\ 1.5 & 6100 & 35 & 296 \\ 1.5 & 6300 & 32 & 270 \\ 1.5 & 3500 & 35 & 281 \\ 2.5 & 2200 & 7 & 77 \\ 2.0 & 1000 & 12 & 166 \\ 2.5 & 3800 & 17 & 178 \\ 1.5 & 16500 & 20 & 225 \\ 2.5 & 400 & 7 & 105 \\ 2.0 & 400 & 7 & 104 \\ 2.0 & 800 & 7 & 92 \\ 2.5 & 500 & 7 & 93 \\ 2.5 & 600 & 12 & 172 \\ 2.0 & 2000 & 17 & 232 \\ 1.0 & 5400 & 20 & 156 \\ 2.0 & 10800 & 12 & 187 \\ 2.0 & 300 & 10 & 130 \\ 2.0 & 400 & 7 & 117 \\ 6.0 & 900 & 12 & 173 \\ 6.5 & 1000 & 15 & 155 \\ & & & \end{array}$

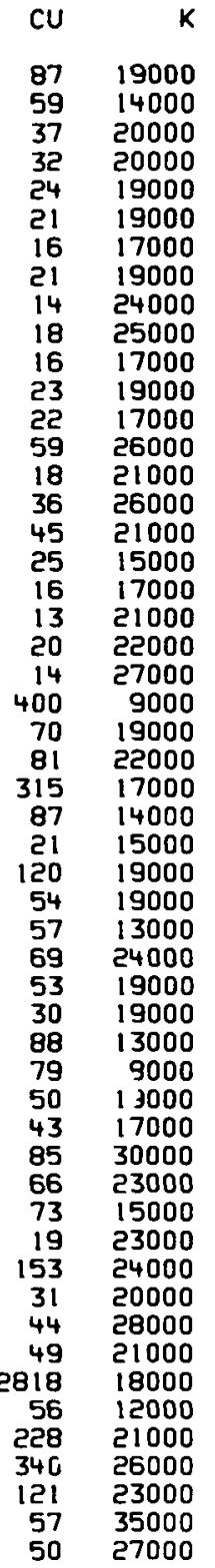




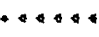

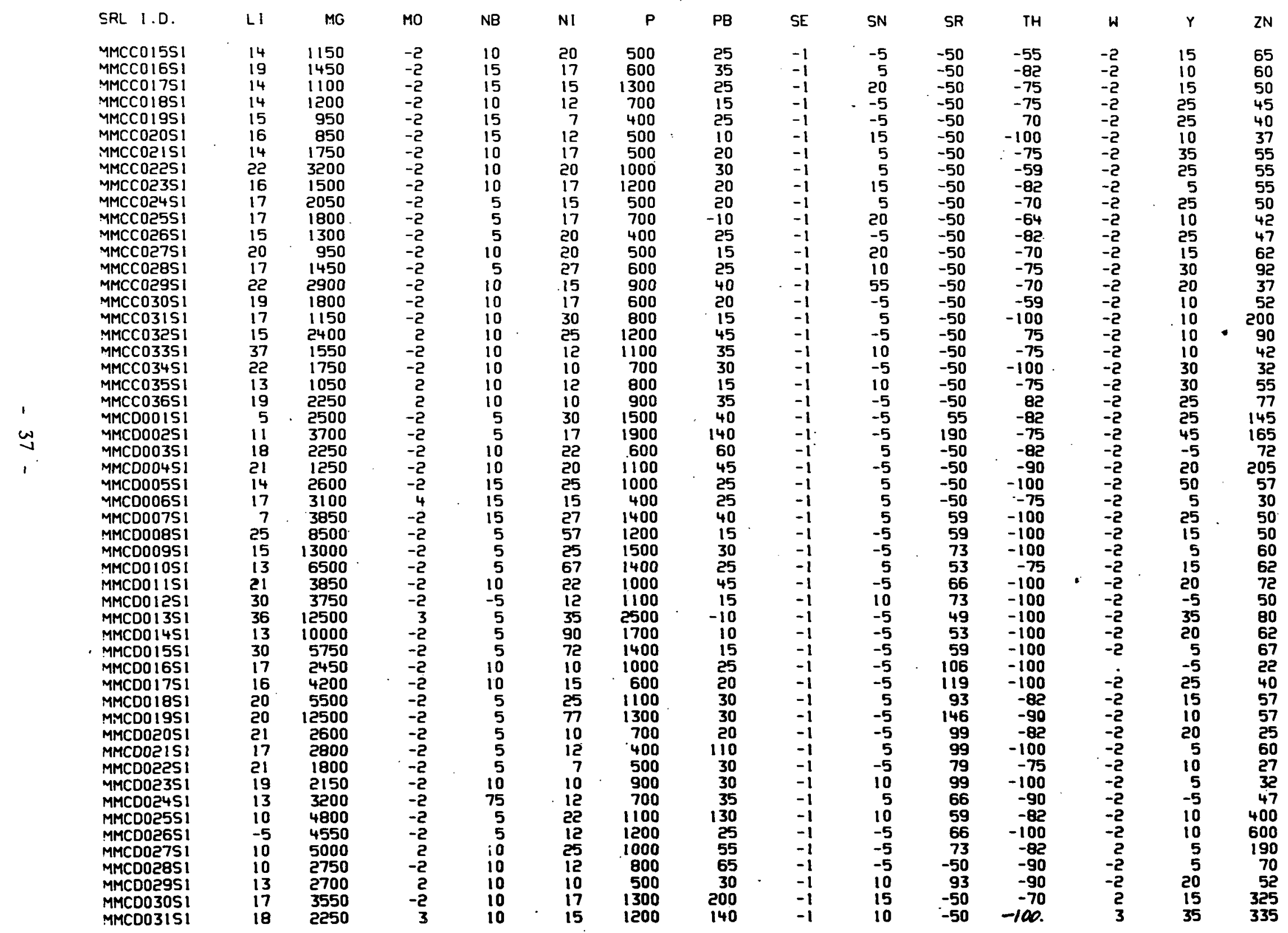

CONCENTRATIONS REPORTED IN INEIGHT PARTS PER MILLION (PPM). 
SRL $1 . D$.

DOE I.D.

MMCDO325: $04-33.4084-111.2052-4-59-000$ MC $0015: 04-33.3636-110.9384-4-59-000$ YMCEDO2S: 04-33.3427-110.9352-4-59-000 YMCE00351 04-33.3179-110.9228-4-59-000 YMCE00451.04-33. $3451-110.9890-4-59-000$ $4 M C 0451.04-33.3451-110.9890-4-59-000$ YMCEOOS . $04-33.380=-110.8960-4-59-000$ $04-33.4805-110.8784-4-59-000$ MMCEO08SI $04-33.4735-110.9072-4-59-000$ MMCEO09S1 04-33.4731-110.9409-4-59-000 MMCEOiDS1 04-33.4628-110.9272-4-59-000 MMCEOIIS1 $04-33.4363-110.8969-4-59-000$ YMCE012S1 $04-33.41011-110.9277-4-59-000$ YMCE013S1 $04-33.4076-110.9060-4-59-000$ YMCE01451 04-33.2782-110.8885-4-59-000 YMCEO15S1 $04-33.2655-110.9071-4-59-000$ YMCEDITSI $04-33.389 .110 .9494-4-59-000$ YMCEOIBS1 04-33.399i-110.9653-4-59-000 YMCEOISSI $04-33$. YMCE020S1 $04-33.4153-110.7795-4-59-000$ YMCEOPIS! $04-33.4415-110.7581-4-59-000$ YMCEOD2S1 $04-33.475-110.7581-4-59-000$ YMCEOC25! 04-33.47E3-110.7538-4-59-000 MMCEOE3S1 04-33.3334-110.7614-4-59-000 MMCEOC4SI $04-33.3076-110.7780-4-59-000$ MMCEOCSSI 04-33.3858-110.7571-4-59-000 MMCE026S1 04-33:3861-110.7916-4-59-000 MCEUC751 04-33.37i6-110.8163-4-59-000 MMCEOC8S1 04-33.388,-110.8600-4-59-000 MMCEO29S1 $104-33.4393-110.8518-4-59-000$ YMCEO30SI $04-33.4431-110.8165-4-59-000$ MMCE031S1 04-33.4781-110.8529-4-59-000 MMCE032S! $04-33.469+110.8019-4-59-000$ MMCE033S1 $04-33.44: 1-110.7817-4-59-000$ YMCE034S1 $04-33.40: 0-110.8360-4-59-000$ MMCE 03551 04-33.3513-110.8043-4-59-000 MMCE03651 $04-33.3323-110.8411-4-59-000$ MMCE037S1 $04-33.3016-110.8580-4-59-000$ MMCE038S1 04-33.2709-110.8511-4-59-000 MMCE039S1 $04-33.3085-110.7943-4-59-000$ MMCEO40S1 04-33.2633-110.8070-4-59-000 MMCEC4IS1 04-33 $2774-110.7527-4-59-000$ MMDAODIS1 04-33.2343-111.7767-4-59-000 MMDAOD251 $04-33.23=8-111.8137-4-59-000$ MMDAOOSI $04-33.2388-111.8737-4-59-000$ MMDAOOS1 04-33.2319-111.8701-4-59-000 MMDAOOSI $04-33.1549-111.7749-4-59-000$ MMDAOO5S1 04-33.13:9-111.7708-4-59-000 MMDAO06.51 04-33.1374-111.8147-4-59-000 MMDA007.51 $04-33.1588-111.8600-4-59-000$ MMDAOOBSI 04-33.1312-111.8527-4-59-000 MMDA009' 1 04-33.2039-!11.7602-4 59-000 MMDA010'51 04-33.2001-\$11.8333-4-59-000 MMDAO1151 04-33.1864-111.8128-4-59-000
CONCENTRATIONS REPORTED IN WEIGHT PARTS PER MILLIDN (PPM).

\begin{tabular}{ll}
$U X$ & $A G$ \\
8.4 & 0.1 \\
0.8 & 0.2 \\
0.8 & 0.1 \\
3.6 & 0.8 \\
0.8 & 0.5 \\
0.8 & 0.2 \\
4.4 & 0.3 \\
0.5 & 0.9 \\
0.5 & 0.3 \\
1.5 & 0.4 \\
1.4 & 0.1 \\
3.5 & 0.4 \\
1.5 & 0.3 \\
1.5 & 0.2 \\
0.8 & 0.9 \\
0.8 & 0.1 \\
0.5 & 0.7 \\
0.9 & 0.2 \\
0.7 & 0.8 \\
1.2 & 1.6 \\
0.4 & 1.6 \\
0.6 & 0.5 \\
0.6 & 0.8 \\
1.1 & 0.7 \\
0.3 & 1.0 \\
0.6 & 0.6 \\
0.7 & 0.5 \\
0.6 & 0.6 \\
0.4 & 0.6 \\
1.3 & 0.2 \\
0.4 & 0.3 \\
0.5 & 0.2 \\
0.2 & 0.3 \\
0.8 & 1.9 \\
0.4 & 0.6 \\
0.7 & 0.3 \\
1.1 & 0.5 \\
0.3 & 0.6 \\
1.4 & 0.4 \\
0.8 & 0.3 \\
0.2 & 0.2 \\
0.7 & 0.2 \\
1.7 & 0.5 \\
0.4 & 0.4 \\
0.8 & 0.5 \\
0.2 & 0.5 \\
1.7 & 0.4 \\
1.3 & 0.4 \\
0.7 & 0.7 \\
0.6 & 0.2 \\
0.4 & 0.2 \\
0.5 & 0.3 \\
0.4 & 0.2 \\
& \\
\hline
\end{tabular}

\section{BE}

5.5

4.0

3.0

3.5

3.5

4.0

4.0

2.
2. 5

5.0

3.0

4.5

3.0

3.5

3.0

1.5

1.5
3.0

1. 5

2.5

2.0

1.5

2. 5

1.5
2.0

2. 0

1.5

2.

2. 0

2.

1.0
35.5

2.5

2.5
2.0
2.0

2.0
1.5

2.5

2.

2.

1.5

2.

2. 0

2. 0

2.

1.5

2. 0

2. 0

1.5
1.5

$\begin{array}{rrr}\text { CR } & \text { CU } & K \\ & & K \\ 180 & 34 & 30000 \\ 78 & 104 & 17000 \\ 249 & 75 & 24000 \\ 209 & 453 & 21000 \\ 103 & 88 & 21000 \\ 185 & 176 & 25000 \\ 165 & 993 & 25000 \\ 287 & 54 & 12000 \\ 229 & 175 & 13000 \\ 187 & 140 & 30000 \\ 160 & 336 & 30000 \\ 231 & 493 & 30000 \\ 193 & 219 & 28000 \\ 121 . & 568 & 21000 \\ 180 & 113 & 31000 \\ 185 & 45 & 24000 \\ 151 & 52 & 14000 \\ 318 & 91 & 7000 \\ 184 & 125 & 12000 \\ 386 & 2947 & 13000 \\ 162 & 1927 & 12000 \\ 196 & 272 & 14000 \\ 160 & 147 & 28000 \\ 320 & 77 & 23000 \\ 237 & 56 & 11000 \\ 324 & 512 & 10000 \\ 349 & 205 & 10000 \\ 310 & 68 & 10000 \\ 369 & 432 & 9000 \\ 255 & 642 & 18000 \\ 339 & 447 & 13000 \\ 240 & 337 & 10000 \\ 328 & 152 & 7000 \\ 262 & 417 & 8000 \\ 221 & 827 & 9000 \\ 379 & 126 & 14000 \\ 238 & 45 & 11000 \\ 283 & 95 & 11000 \\ 209 & 60 & 21000 \\ 281 & 51 & 16000 \\ 231 & 56 & 10000 \\ 213 & 31 & 16000 \\ 180 & 31 & 17000 \\ 213 & 19 & 12000 \\ 209 & 22 & 14000 \\ 167 & 41 & 20000 \\ 190 & 54 & 11000 \\ 189 & 30 & 12000 \\ 196 & 25 & 15000 \\ 166 & 47 & 12000 \\ 151 & 17 & 17000 \\ 178 & 19 & 20000 \\ 166 & 21 & 18000\end{array}$


$\ldots$

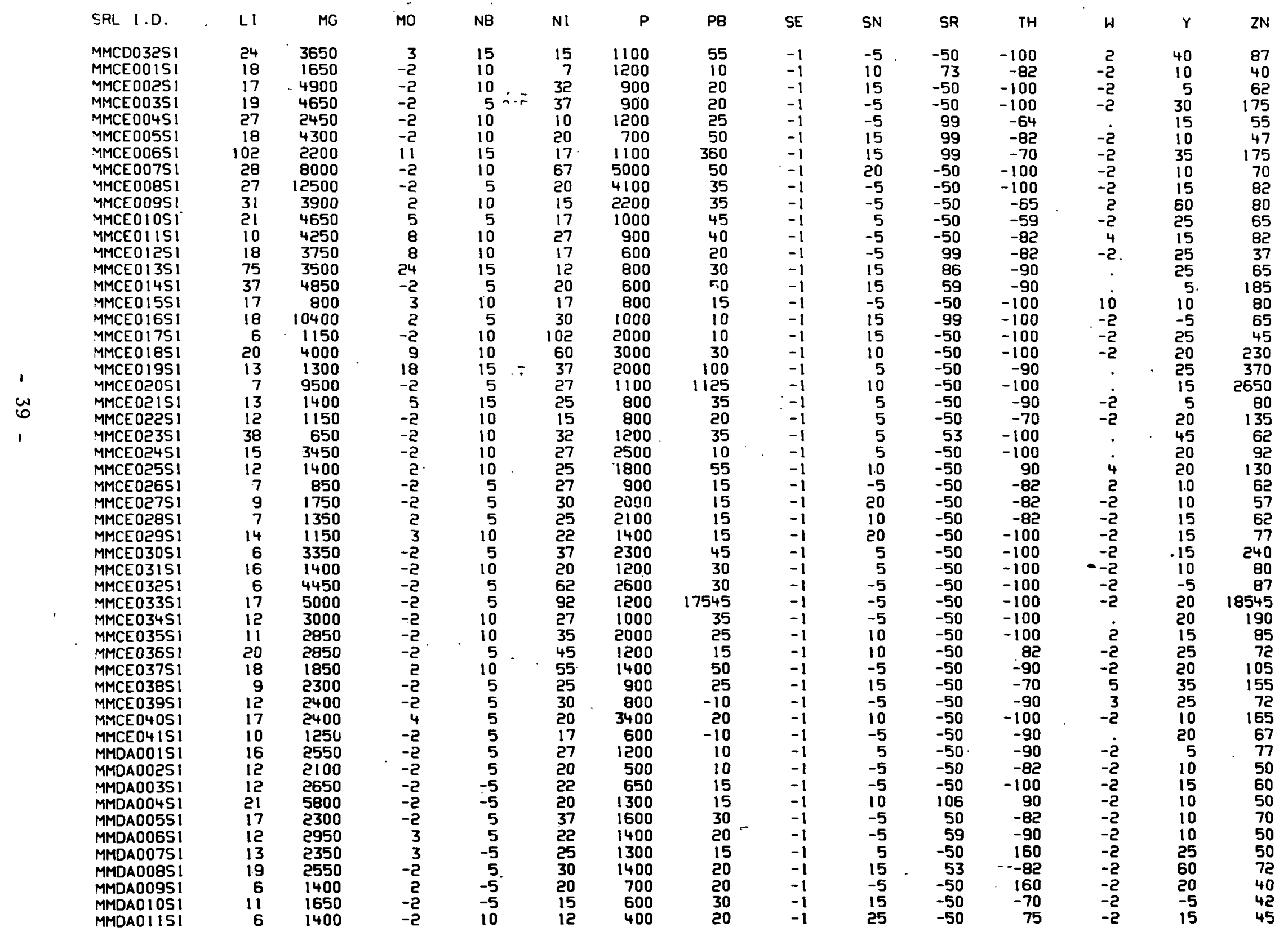

CONCENTRATIONS REPORTED IN WEIGHT PARTS PER MILLION (PPM). 
$+\cdots+\cdots$

DOE 1.D.

MMDA012S1 04-33.1709-111.7941-4-59-000 MMDA01351 04-33.2202-111. 9126-4-59-000 YMDAOI5S1 04-33.2344-11 $04-33.2007-111.9774-4-59-000$ MMDA01751 04-33.1704-111. 9774-4-59-000 (25) $04-33.1906-111.9342-4-59-000$ YMDA019S: 04-33.1908-111. 4MDAO20S: $04-33.1725-111.0260-4-59-000$ YMDAOCOS: 04-33.1725-111.9260-4-59-000 MMOADEISI $04-33.1715-111.8874-4-59-000$ MMOAOE2SI 04-33.0665-111.8484-4-59-000 MMDA02351 04-33.0764-111.8985-4-59-000 MMOAOE4S1 04-33.0469-111.9257-4-59-000 MMDA02751 $04-33.088-1 i 1.9271-4-59-000$ MMOA02851 04-33.1189-111.9823-4-59-000 MMDA029SI 04-33.1203-111.9364-4-59-000 YMDA030S1 04-33.1187-111.8991-4-59-000 MMDA031S: $04-33.0533-111.7892-4-59-000$ MMDA032Si $04-33.0668-111.8154-4-59-000$ MMDA03351 04-33.0880-111.7686-4-59-000 MMOA03451 04-33.0965-111.7998-4-59-000 MMDBO0251 04-33.2493-111.6858-4-59-000 4MDBO0451 04-33.2090-111.7272-4-59-000 4MDBD0551 04-33.2041-111.6815-4-59-000 MMD800651 04-33.2023-111.6336-4-59-000 MMDBD0751 04-33.1714-111.6330-4-59-000 YMDBD08S1 04-33.1744-111.6705-4-59-000 4MDBDogs1 04-33.0755-111.7195-4-59-000 MMOOIS1 04-33.0743-111.6920-4-59-000 MMDBDII $04-33.0847-111.6527-4-59-000$ MMDO 04-33.0753-111.6113-4-59-000 MMBO1 04-33.0759-11.6113-4-59-000 MMDBO1 351 04-33.1089-111.6705-4-59-000 MMDOL 451 04-33.1278-111.6458-4-59-000 MMDBOI5S1 04-33.1302-111. 7282-4-59-000 MMOBOL6S1 04-33.1457-111.7168-4-59-000 MMOBOI7S1 04-33.0554-111.6817-4-59-000 MMDBDI8S1 04-33.0499-111.7294-4-62-000 MMDBC 19S1 04-33.0186- $111.7420-4-59-000$ MMDBO20S1 04-33.0232-1 $11.6707-4-59-000$ MMDBO21S1 04-33.0508-111.6417-4-59-000 MMDBD22'51 04-33.0369-111.6176-4-59-000 MMDB02351 04-33.0320-111.5591-4-59-000 MMDBO24 $04-33.0589-111.5586-4-59-000$ MMOB025S1 $04-33.0544-111.5254-4-59-000$ MMOB02651 $04-33.0020-111.5239-4-59-000$ $04-33.0256-111.5948-4-59-000$ $04-33.0758-111.5461-4.59-000$ MMCB020 MMOBOE9S1 $04-33.0884-111.5286-4-59-000$
COMCENTRATIONS REPORTED IN WEIGHT PARTS PER MILLION (PPM).

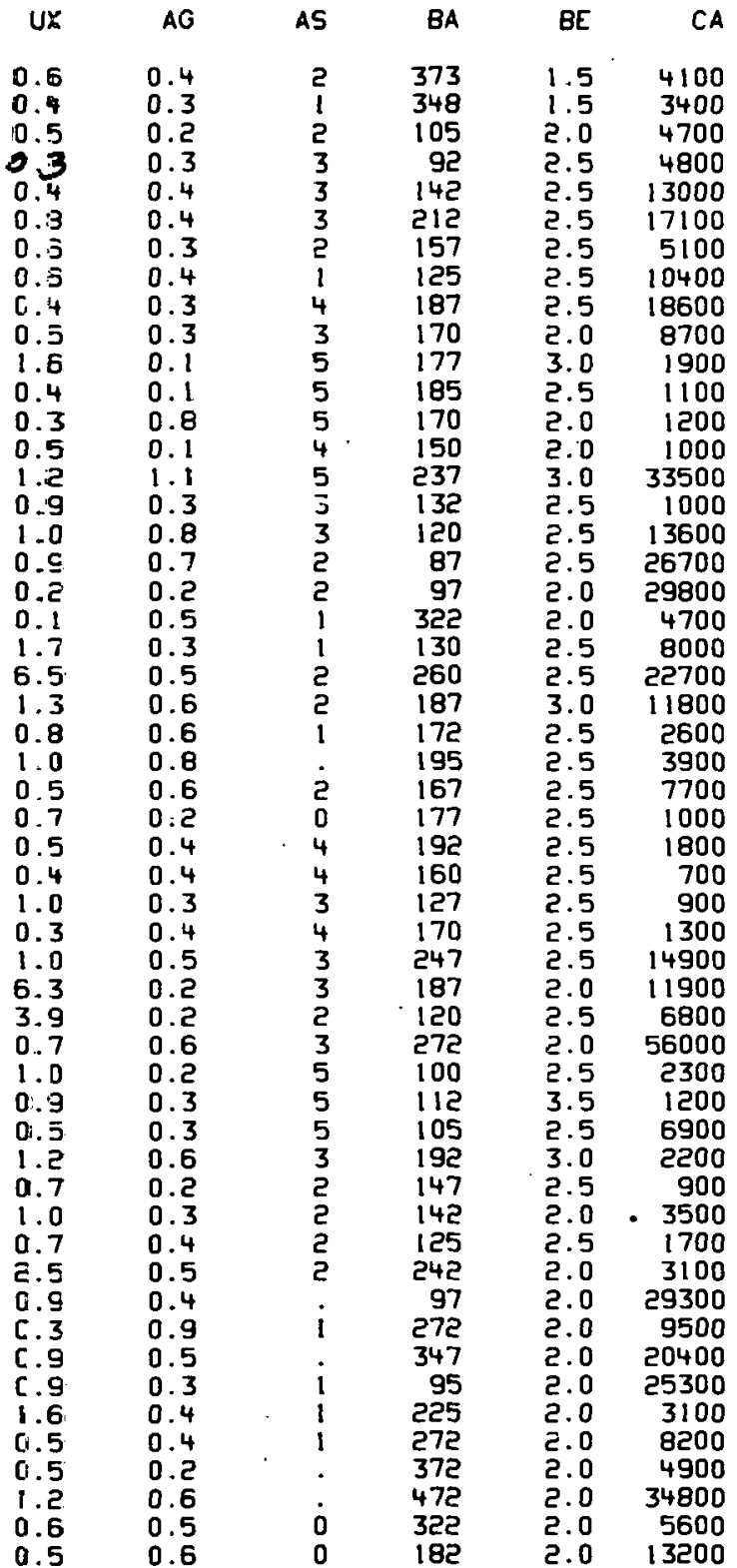

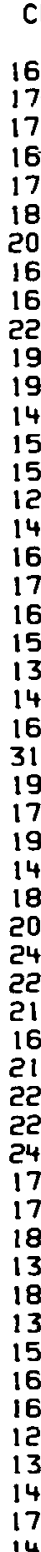

167
167
174
173
168
175
181
203
168
167
222
195
190
149
150
154
125
148
168
177
161
158
136
142
166
311
197
175
194
145
181
207
245
226
215
167
214
223
222
240
174
176
187
133
186
130
153
162
166
121
132
147
179
143
4. 16000 $16 \quad 25000$ 1424000 5422000 $18 \quad 22000$ 1824000 $17 \quad 21000$ 2423000 $\begin{array}{ll}23 & 24000 \\ 22 & 24000\end{array}$ 1852000 $72 \quad 21000$ $17 \quad 24000$ $\begin{array}{ll}20 & 22000 \\ 28 & 21000\end{array}$ 1820000 $25 \quad 21000$ $24 \quad 19000$ $32 \quad 24000$

3124000

41
19 24000

$\begin{array}{ll}33 & 24000\end{array}$ $27 \quad 21000$ $32 \quad 22000$ ट2 22000 
+4

SRL 1.0

MMDAOISS MMDA0 1351

MMOAO 14S

MMDAO15S

MMDAO 1651

MMDAO 1751

MMDAOISSI

MMDAOISI

MMDAOEOSI

MMDAOEIS!

MMDAO2351

MMDA024S1

MMDA025SI

MMDAO2651

MMDA02751

MMDAO285

MMDA030SI

MMOAD31S

MMDA0325!

MMDA03351

MMDA03451

MMDBOOISI

MMDBO02SI

MMDB00351

MMDBD0451

MMDB00551

MMOBO0751

MMDBO0BS1

MMOB00951

MMDBO 1051

MMDBOIISI

MMDBO I2SI

MMDBO 1351

MMDBO 1451

MMDBO 15S1

MMDBO 16S1

MMDBO 1751

MMDBO 1851

MMDBO ISSI

MMOB020S1

MMDB021S1

MMDB02251

MMDBO2351

MMD802451

MMDB025SI

MMDB02651

MMDB02751

MMDB02851

MMDB0295!

MMDB030S1
CONCENTRATIONS REPORTED IN WEIGHT PARTS PER MILLION (PPM).

$\begin{array}{rrr}\text { LI } & M G & M O \\ & & \\ 7 & 1300 & -2 \\ 11 & 2550 & 2 \\ 16 & 4700 & -2 \\ 13 & 4500 & -2 \\ 19 & 7500 & -2 \\ 15 & 6000 & 2 \\ 9 & 3900 & -2 \\ 12 & 4700 & -2 \\ 24 & 5000 & -2 \\ 12 & 3400 & -2 \\ 22 & 2900 & -2 \\ 14 & 2700 & -2 \\ 14 & 3500 & -2 \\ 14 & 3000 & -2 \\ 34 & 7500 & -2 \\ 12 & 3650 & -2 \\ 13 & 4150 & -2 \\ 28 & 7100 & -2 \\ 20 & 5600 & -2 \\ 13 & 4250 & -2 \\ 12 & 3250 & -2 \\ 19 & 5250 & -2 \\ 13 & 3250 & -2 \\ 18 & 4200 & -2 \\ 12 & 3300 & -2 \\ 19 & 4350 & -2 \\ 12 & 3050 & -2 \\ 12 & 2500 & -2 \\ 16 & 2850 & -2 \\ 10 & 3100 & -2 \\ 18 & 2850 & -2 \\ 12 & 3500 & -2 \\ 13 & 3500 & -2 \\ 15 & 2650 & -2 \\ 15 & 3000 & -2 \\ 14 & 3100 & -2 \\ 15 & 2800 & -2 \\ 8 & 2000 & -2 \\ 12 & 2450 & -2 \\ 11 & 2600 & -2 \\ 13 & 3050 & -2 \\ 11 & 3200 & -2 \\ 13 & 1950 & -2 \\ 22 & 4400 & -2 \\ 12 & 2750 & -2 \\ 15 & 2900 & -2 \\ 15 & 4500 & -2 \\ 16 & 3000 & -2 \\ 14 & 2750 & 2 \\ 14 & 1900 & -2 \\ 15 & 2700 & -2 \\ 16 & 2250 & -2 \\ 17 & 3800 & 2 \\ & & \end{array}$

NI

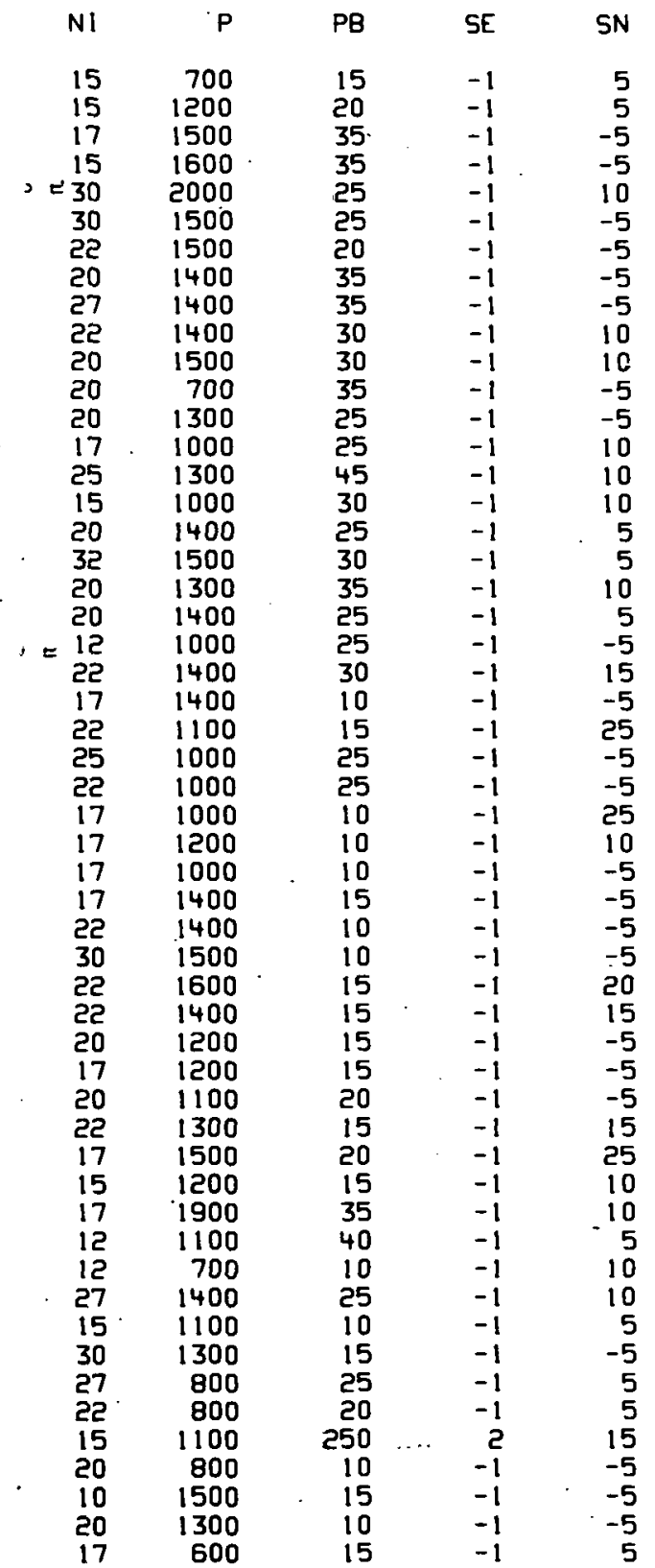

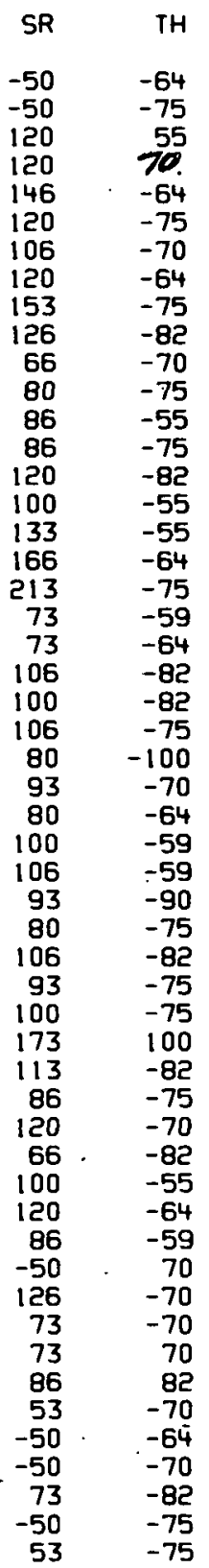


SRL 1.0 .

DOE I.D.

MMDB031S1 $04-33.1234-111.5565-4-59-000$ MMDi303251 04-33.1236-111.5929-4-59-000 YMDB03351 04-33.1465-111.5139-4-59-000 $4 M 0335104-33.1465-111.5139-4-59-000$ 4MB03451 04-33. $1614-111.5637-4-59-000$ MMDO3551 04-33.1636-111.6159-4-59-000 MMDO365l . 04-33.1980-111.6103-4-59-000 MMOBO37S1 04-33.2045-111.5334-4-59-000 YMOB039S1 $04-33.2302-111.5272-4-59-000$ MMDBO40S1 $04-33.1980-111.5635-4-59-000$ MMDB04ISI $04-33.0262-111.6395-4-59-000$ MMDC001S1 $04-33 \cdot 1437-111.4954-4-59-000$ YMDCO02S1 $04-33 \cdot 1647-111.4953-4-59-000$ YMOC003S1 $04-33.1900-111.4785-4-59-000$ $4 M 0 C 00451 \quad 04-33 \cdot 1606-111.4377-4-59-000$ YMDC 005S1 04-33.1324-111.4010-4-59-000 YMDC00651 04-33.2302-111.4738-4-59-000 MMDCD0751 04-33.2288-111.4299-4-59-000 YMDC00851 04-33.2267-111.4057-4-59-000 MMDCDO9S1 04-33.0032-111.4713-4-59-000 YMDCO10S1 04-33.0159-111.4245-4-59-000 MMDCDIIS1 04-33.0533-111.3940-4-59-000 MMDCDIวS1 04-33.0500-111. 4351-4-59-000 MMOCDI3Si $04-33.0465-111.4764-4-59-000$ MMDCD 1451 04-33.0185-11. 3925-4-59-000 MMDCDISS1 $04-33.1001-111.4034-4-59-000$ MMOCDI5S1 04-33.1001-111.4034-4-59-000 YMOCDS1 $04-33.1001-111.4034-4-59-000$ 4MCO1651 04-33.0784-111.4218-4-59-000 MMDC 1751 04-33.0988-111.4765-4-59-000 04-33.1252-111.4367-4-59-000 MMDCOISS1 04-33.1183-111.3605-4-59-000 MMOCD20S1 $04-33 \cdot 1612-111.3552-4-59-000$ MMDCDISl $04-33 \cdot 1484-111.3834-4-59-000$ MMDCD235I 04-33.1895-111.3850-4-59-000 MMDCO2451 04-33.1910-111.4365-4-59-000 MMDC025S1 04-33.2165-111.3602-4-59-000 MMOC02651 04-33.2319-111.2991-4-59-000 MMCC02751 $04-33.2443-111.2840-4-59-000$ MMCC2751 04-33.1883-111.3246-4-59-000 MMDCO2951 04-33.1916-111. MMCC2951 04-33.1983-111. MMCO3051 04-33.181-111.2845-4-59-000 MMOCO3151 04-33.1543-11. MMOCO3151 04-33.1543-111.2987-4-59-000 MMCC03esi 04-33.1280-111.3060-4-59-000 MMDCO3351 04-33.0966-111.3003-4-59-000 MMDCO34SI 04-33.1043-111.2715-4-59-000 MMOCO35S1 04-33.1247-111.2583-4-59-000 MMOC03651 04-33.0329-111. MMDC03751 04-33.0115-111.3082-4-59-000 MMOC03851 04-33.0040-111.2828-4-59-000 MMDC03951 04-33.0608-111.3189-4 59-000 MMDC04051 04 MMDC 04151 0.1-33.0552-111.3624-4-59-000
CINCENTRATIONS REPORTED IN WEIGHT PARTS PER MILLION (PPM).

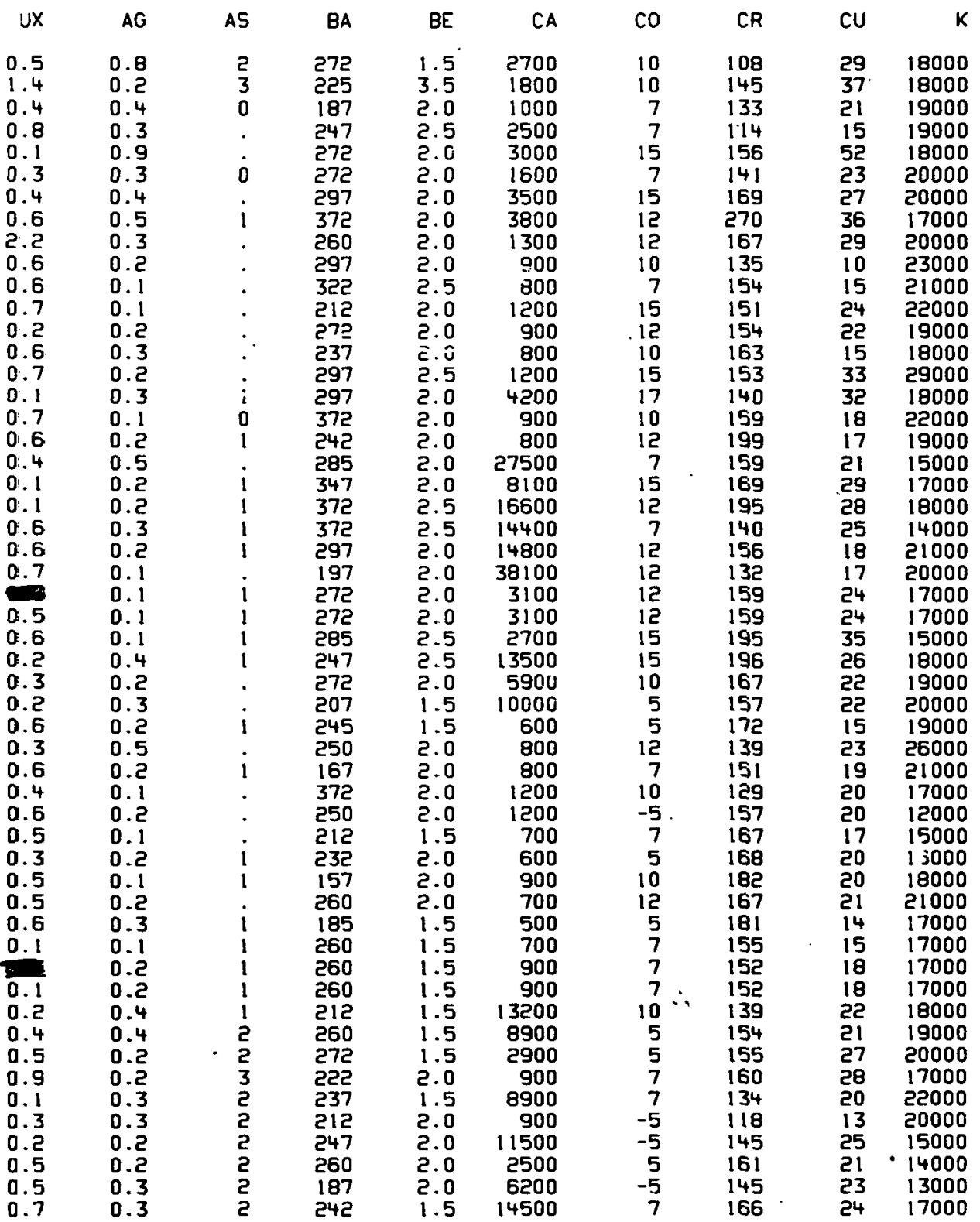


.0.0.4. MMDBO32S1

MMDB03351

MMO803451

MMDB035S!

MMOB036S!

MMDB0375

MMDB03951

MMOB040S!

MMDBO4151

MMOCDOIS

MMDC003S

MMDC004S1

MMOC005SI

MMOC0065

MMDCOO7S

MMDC0085

MMC009S

MMDCOIOS

MMDCOIIS

MMDCOIZS

\& MMDCOI3S

MMDCO14S

MMDCO15S

MMDCOLISI

MMDCOI6SI

MMOCO175

MMOCOI8S

MMDCOIOS

MMDC020S

YMOCO2151

MMDCDE2S

YMDCO235

MMDCOZ4S

MMDCDSSI

MMDC 02651

MMDC027S

MMDC028S1

MMDC029S

MMOC03051

MMDC031S!

MMDC031S1

MMDC032SI

MMDC0335

MMDC034S

MMDCO35S

MMDC 03651

MMDC037S1

MMDC038S

MMOC039S

MMDCO40SI

MMDC 04151

$\begin{array}{rrr}L 1 & M G & M O \\ 18 & 2550 & -2 \\ 23 & 2300 & 2 \\ 17 & 1900 & -2 \\ 18 & 2100 & 2 \\ 26 & 2850 & -2 \\ 16 & 2000 & -2 \\ 19 & 2800 & -2 \\ 12 & 1750 & -2 \\ 19 & 1900 & -2 \\ 13 & 1350 & 2 \\ 20 & 1550 & -2 \\ 20 & 2450 & -2 \\ 21 & 2500 & -2 \\ 19 & 1850 & 2 \\ 27 & 3150 & -2 \\ 20 & 3000 & 2 \\ 19 & 1900 & -2 \\ 20 & 1900 & -2 \\ 14 & 3150 & -2 \\ 15 & 2350 & -2 \\ 13 & 2100 & -2 \\ 14 & 2750 & 2 \\ 13 & 2650 & 3 \\ 12 & 3200 & -2 \\ 15 & 2700 & -2 \\ 15 & 2700 & -2 \\ 15 & 2350 & 2 \\ 13 & 2500 & 2 \\ 16 & 2450 & -2 \\ 11 & 2250 & -2 \\ 13 & 1300 & -2 \\ 22 & 2600 & -2 \\ 17 & 1500 & -2 \\ 22 & 2100 & 2 \\ 15 & 2250 & 2 \\ 12 & 1750 & 3 \\ 16 & 1600 & 2 \\ 14 & 2150 & -2 \\ 17 & 3300 & -2 \\ 16 & 1450 & -2 \\ 17 & 1500 & 2 \\ 13 & 3000 & 3 \\ 13 & 3000 & 3 \\ 10 & 1650 & 2 \\ 8 & 1750 & -2 \\ 12 & 2400 & 2 \\ 15 & 1350 & 2 \\ 12 & 2150 & 3 \\ 14 & 2900 & -2 \\ 12 & 2500 & -2 \\ 10 & 3250 & -2 \\ 13 & 2550 & -2 \\ 14 & 2750 & -2 \\ & & \end{array}$

NB

15
10

75

40
25

25

15

25

10

10

15

15

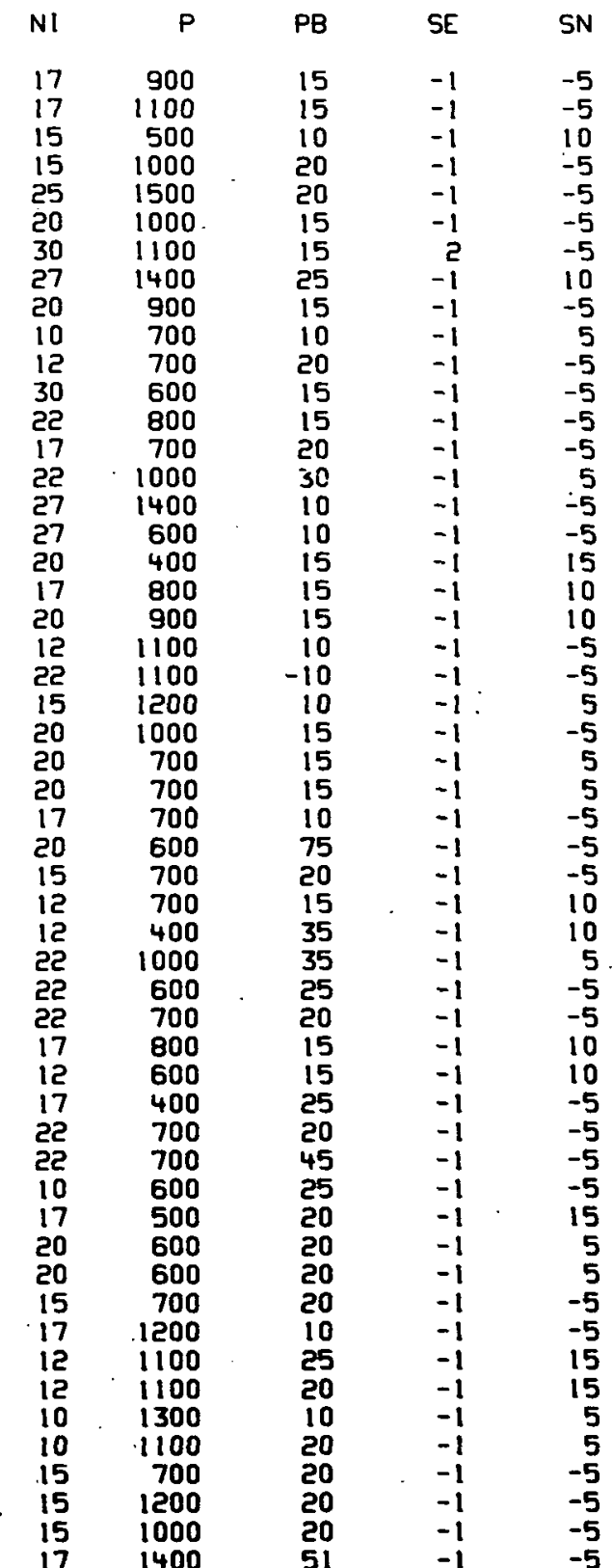

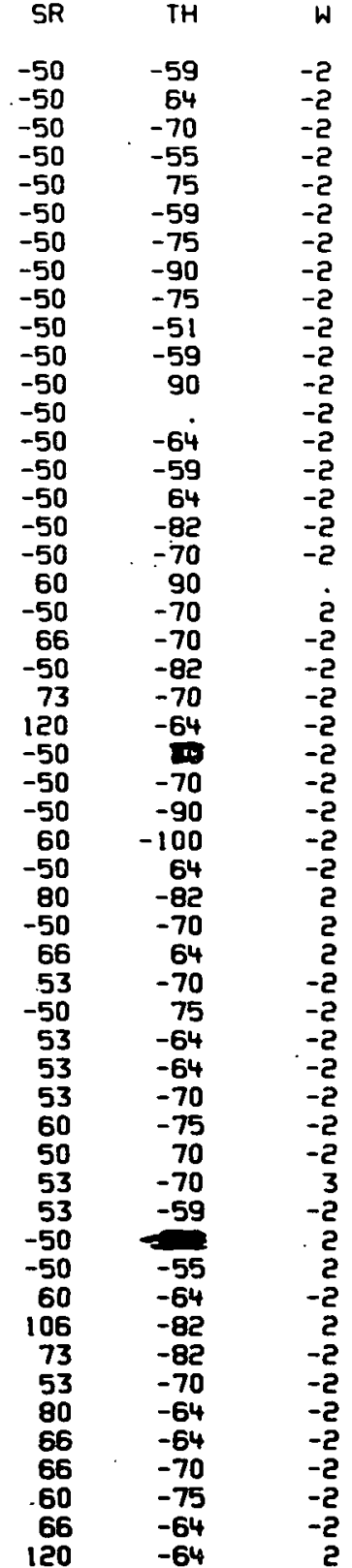




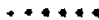

SRL $1 . D$.

DOE I.D.

MMDC042S1 $04-33.0798-111.3727-4-59-000$ MDJO0IS1 04-33.1955-111.2418-4-59-000 4MDDO02S1 04-33.2253-111.2238-4-59-000 MMDD0035L 04-33.2018-111.1963-4-59-000 MMDDO0451 04-33.2145-111.1970-4-59-000 MMDD005S1 $04-33.207 i-111.1536-4-59-000$ MMDD006S1 $04-33.2193-111.1480-4-59-000$ MMDD00751 04-33.1620-111.2021-4-59-000 YMDD0085: $04-33 \cdot 1653-111.1613-4-59-000$ YMDD00951 04-33.1317-111.2012-4-59-000 4MDDO1051 04-33.1089-111.2135-4-59-000 YMDDOIIS1 $04-33,103 i-111.2478-4-59-000$ YMDDDIXS1 04-33. YMDDOI3S1 04-33.0445-111. YMDOD 45 I 4MODO1451 04-33.0236-111.2155-4-59-000 MMDOI5S1 04-33.0378-11..1999-4-59-000 MMDDI651 04-33.0196-111.1860-4-59-000 MMDDOIIS1 04-33.0168-111. 1586-4-59-000 4MODOI8S1 04-33.0632-111. MMDDOI9S1 04-33.0958-111.1389-4-59-000 MMDDOCOS1 04-33.1083-111.1486-4-59-000 MMDOD2151 04-33.0328-11.1.1172-4-59-000 MMDDO22S1 04-33.0443-111.1048-4-59-000 MMDD02351 04-33.0509-111.0683-4-59-000 4MDD024S: 04-33.0730-111.0888-4-59-000 MMDD025S! 04-33.0859-111.0770-4-59-000 MMDD0265: $04-33.112 z-111.0865-4-59-000$ MMDD0275: $04-33.0087-111.0626-4-59-000$ MMDDD28S ! 04-33.2343-111.0950-4-59-000 YMDD029S $104-33.2369-111.0536-4-59-000$ MMDD030S1 $04-33.1925-111.0468-4-59-000$ MMDD0315! $04-33.1805-111.0181-4-59-000$ MMDD03251 $04-33.1605-111.0079-4-59-000$ MMDD03351 04-33.1600-111.0524-4-59-000 MMDD03451 04-33.1345-111. 0654-4-59-000 MMDD035S1 04-33.1254-111.0303-4-59-000 MMDD03751 04-33.0569-111.0344 MMO03751 04-33.0569-111.0344-4. MMODJ81 04-33.0815-111.0132-4-59-000 MMDO03951 04-33.24 MMDDO40S1 04-33.2123-111. $1126-4-59-000$ MMDDC4151 04-33.1454-111. $1054-4-59-000$ MMDD04251 04-33.1461-111.2444-4-59-000 MMDE001S1 04-33.1319-1:0.9807-4-59-000 MMDE00251 04-33.1685-1:0.9732-4-59-000 MMOE00351 04-33.1982-1:0.9923-4-59-000 MMDE004S1 04-33.0900-1:0.9836-4-59-000 MMDE005S1 14 MMDE00751 04-33.1018-110.9475. MMDE00851 04-33.0618-110.8984-4 59-000 MMDED09S1 $04-33.0758-110.8790-4-59-000$ MMDEOIOS1 04-33.0951-110.8703-4-59-000
CONCENTRATIONS REPORTED IN HEIGHT PARTS PER MILLION (PPM).

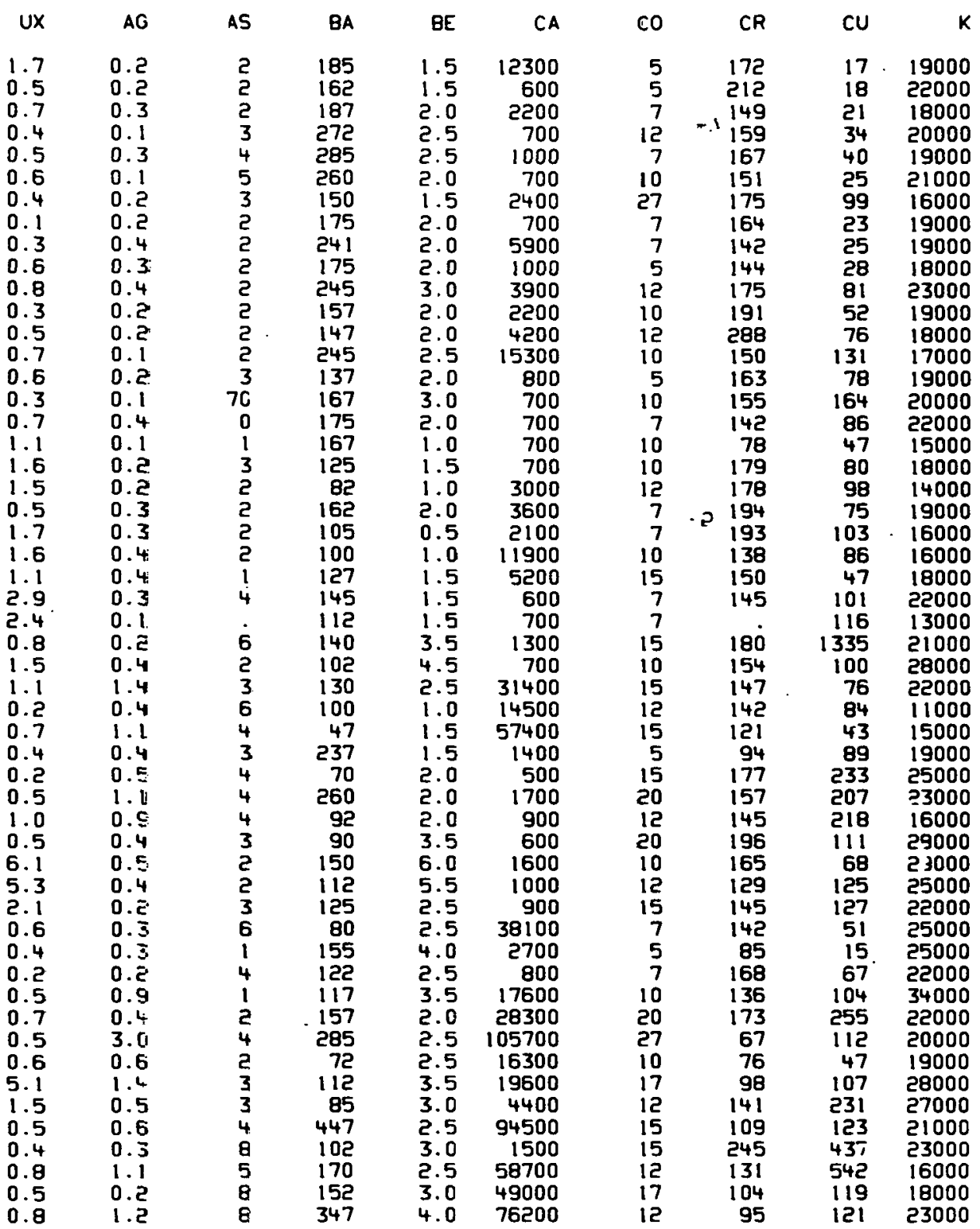


$\ldots+\ldots$

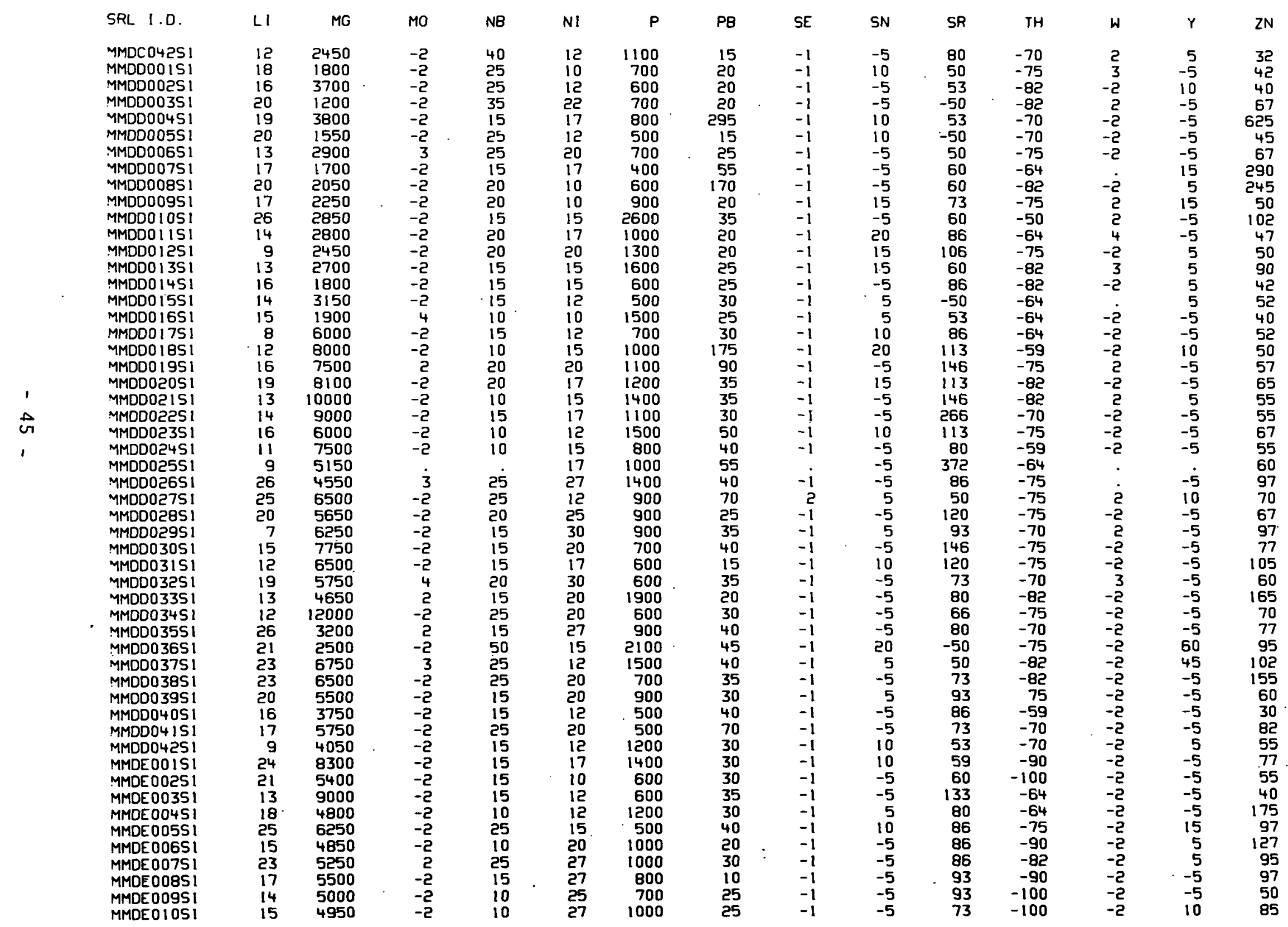

CONCENTRATIONS REPORTED IN WEIGHT PARTS PER MILLION (PPM). 
$\ldots \ldots$

SRL I.D.

COE I.D.

YMDEOIIS.1 $04-33.0177-110.8446-4-59-000$ 'MOLOLI2S.1 04-33.04E9-110.8428-4-59-000 YMDE013S. 04-33.01E6-110.8195-4-59-000 MMDEOI4SI 04-33.0572-110.8036-4-59-000 MMDEOI5SI $04-33.0054-110.7635-4-59-000$ MMDEOI6SI $04-53.3 .1152-110.7696-4-59-1000$ MMDEOI7SI $04-35$ $32.1396-110.8032$ पMOEDट251 04-33. 1578-110.7863-4-59-300 MMDE02351 04-33.1389-110.8554-4-59-300 MMDEOC4S1 04-33.1593-110.8549-4-59-900 MMEO25E1 04-33.1957-110.8452-4-59-000 MMDE026S1 04-33.2304-110.8549-4-59-000 MMDEOC7S1 04-33.1330-110.7956-4-59-000 MMDE029S1 04-33. $302-110.7635-4-59-000$ MMOE030SI $04-33.1827 \cdot 110.8829-4-59-000$ MMDE03151 $04-33.2595-110.8931-4-59-000$ MMDE032S 1 04-33.1780-110.9351-4-59-000 MMDE03351 $04-33.1942-110.9256-4-59-000$ MMDE034S1 D4-33.2351-110.9078-4-59-000 YMDE035S1 04-33.23E5- $110.9337-4-59-000$ YMDE03651 04-33.0749-110.9855-4-59-000
CONCENTRAT IONS REPORTED IN WLIGHT PARTS PER MILL!ON (PPM).

$\begin{array}{rrrrrrrrrr}\text { UX } & \text { AG } & \text { AS } & \text { BA } & \text { BE } & C A & \text { CO } & \text { CR } & \text { CU } & K \\ 0.5 & 1.1 & 4 & 80 & 2.5 & 37600 & 15 & 151 & 250 & 22000 \\ 0.8 & 2.5 & 10 & 397 & 3.0 & 67000 & 22 & 113 & 302 & 22000 \\ 0.1 & 2.9 & 8 & 77 & 1.5 & 14400 & 17 & 190 & 1877 & 22000 \\ 0.3 & 1.1 & 3 & 145 & 2.0 & 2200 & 42 & 196 & 687 & 24000 \\ 0.4 & 2.2 & 6 & 522 & 1.0 & 79000 & 12 & 150 & 2732 & 9000 \\ 0.6 & 1.3 & 1 & 222 & 1.5 & 28200 & 7 & 153 & 567 & 18000 \\ 0.7 & 0.5 & 3 & 117 & 2.0 & 2200 & 12 & 176 & 224 & 23000 \\ 0.4 & 1.1 & 1 & 242 & 1.0 & 7200 & 27 & 175 & 214 & 10000 \\ 0.8 & 1.6 & 6 & 147 & 2.0 & 19200 & 10 & 133 & 136 & 19000 \\ 0.6 & 1.5 & 1 & 622 & 0.5 & 65000 & 22 & 119 & 49 & 6000 \\ 1.3 & 0.6 & 3 & 272 & 2.0 & 1000 & 7 & 141 & 41 & 35000 \\ 2.8 & 1.1 & 3 & 572 & 0.5 & 57200 & 5 & 101 & 37 & 16000 \\ 10.7 & 1.2 & 12 & 150 & 1.5 & 40700 & 12 & 114 & 95 & 21000 \\ 0.1 & 0.2 & 2 & 122 & 1.5 & 29900 & 20 & 185 & 51 & 17000 \\ 0.3 & 0.7 & . & 175 & 1.5 & 4600 & 17 & 194 & 53 & 14000 \\ 0.4 & 1.0 & 1.0 & 107 & 2.0 & 13300 & 30 & 180 & 39 & 24000 \\ 0.4 & 0.3 & 3 & 125 & 1.5 & 2500 & 20 & 174 & 64 & 17000 \\ 1.3 & 0.5 & 1 & 247 & 2.0 & 8300 & 20 & 141 & 54 & 19000 \\ 1.9 & 0.5 & 2 & 212 & 2.0 & 2700 & 12 & 160 & 61 & 20000 \\ 0.7 & 1.0 & 1 & 147 & 1.5 & 21200 & 12 & 200 & 45 & 17000 \\ 0.6 & 1.4 & 6 & 212 & 1.0 & 46200 & 10 & 90 & 27 & 18000 \\ 3.9 & 1.5 & 7 & 45 & 1.0 & 4500 & 5 & 116 & 228 & 19000 \\ 0.2 & 0.3 & 3 & 205 & 1.0 & 23300 & 27 & 171 & 65 & 15000 \\ 1.0 & 0.5 & 3 & 200 & 1.5 & 1300 & 20 & 237 & 52 & 18000 \\ 0.6 & 0.4 & 0 & 100 & 2.5 & 17800 & 25 & 193 & 44 & 17000 \\ 2.8 & 0.3 & 2 & 167 & 2.0 & 500 & 7 & 171 & 113 & 28000\end{array}$


$\cdots+4$

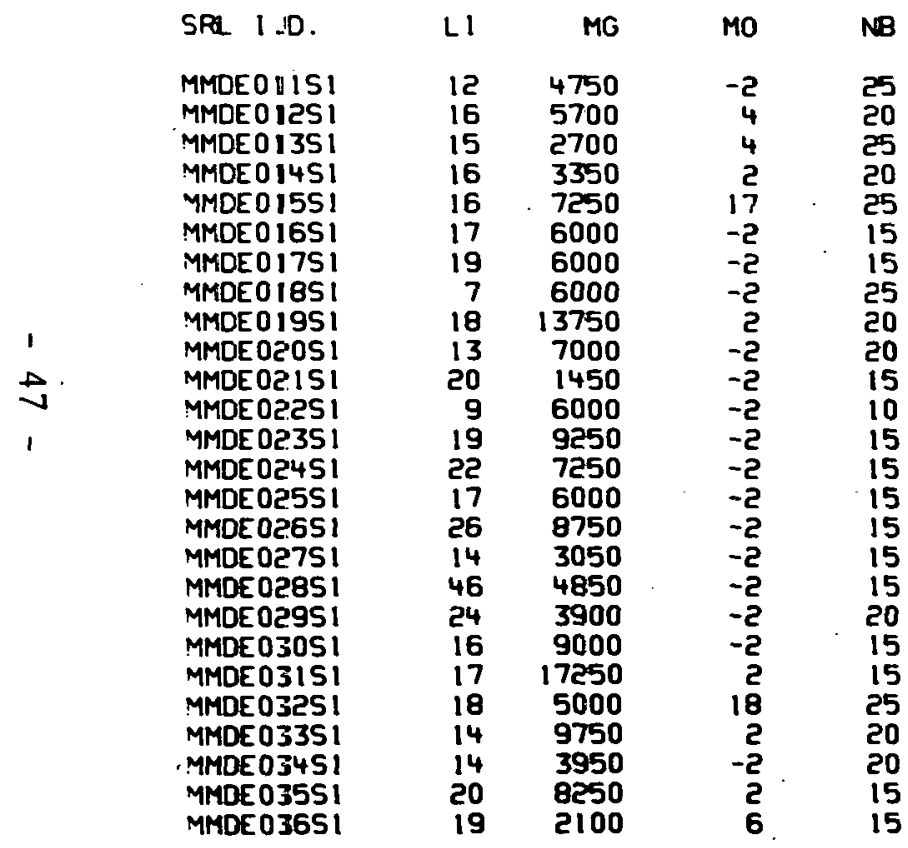

CONCENTRATIONS REPORTED IN WEIGHT PARTS PER MILLION (PPM).

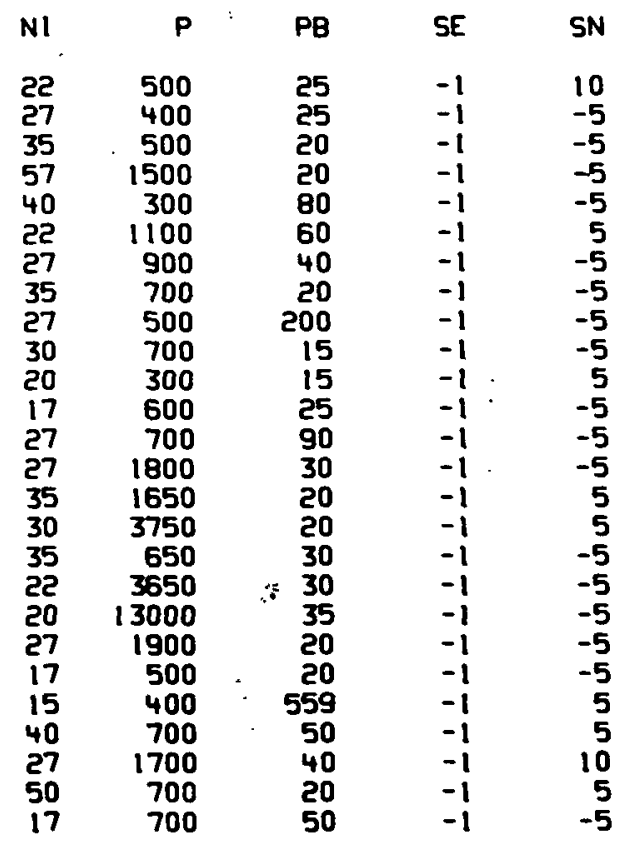

$\begin{array}{rr}S R & T H \\ 93 & -90 \\ 80 & -90 \\ 73 & -82 \\ 73 & -100 \\ 113 & -100 \\ 106 & -90 \\ 133 & -75 \\ 120 & -82 \\ 73 & -82 \\ 93 & -100 \\ -50 & -82 \\ -50 & -90 \\ 73 & -90 \\ 93 & -100 \\ 66 & -100 \\ 66 & -100 \\ 60 & -90 \\ 53 & -90 \\ 53 & -82 \\ 73 & -100 \\ 66 & -82 \\ 53 & -64 \\ 73 & -100 \\ 53 & -100 \\ 100 & -90 \\ 60 & -75\end{array}$

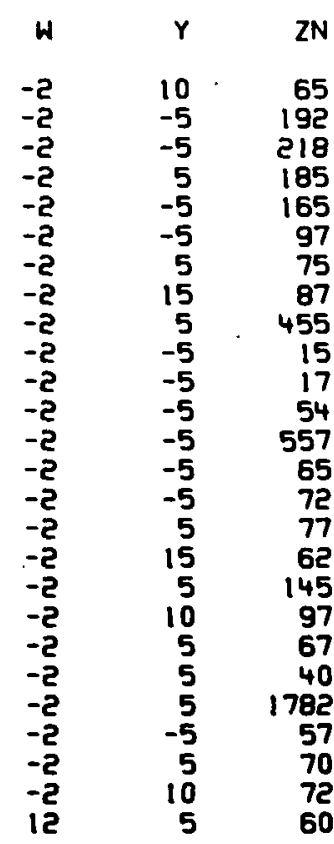

WE. 
THIS PAGE

WAS INTENTIONALLY

LEFT BLANK 


\section{Ground Water Analyses from the Mesa Quadrangle}

Data are tabulated for concentrations of 31 supplementary elements in ground water. The 31 elements are $\mathrm{Ag}, \mathrm{Al}, \mathrm{As}, \mathrm{B}, \mathrm{Ba}$, $\mathrm{Be}, \mathrm{Ca}, \mathrm{Ce}, \mathrm{Co}, \mathrm{Cr}, \mathrm{Cu}, \mathrm{Fe}, \mathrm{K}, \mathrm{Li}, \mathrm{Mg}, \mathrm{Mn}, \mathrm{Mo}, \mathrm{Na}, \mathrm{Nb}, \mathrm{Ni}, \mathrm{P}, \mathrm{Sc}$, $\mathrm{Se}, \mathrm{Si}, \mathrm{Sr}, \mathrm{Th}, \mathrm{Ti}, \mathrm{V}, \mathrm{Y}, \mathrm{Zn}$, and $\mathrm{Zr}$. 
IPPENDIX 11 TABULAIION OF KEY FIELD MEASUREMENTS AND ANALYTICAL DATA---GROUND WATER---

ELEMENTAL CONCENTRATIONS IN PPB. ALK IN MEQ/L. COND IN UM/CM.

\begin{tabular}{|c|c|c|c|c|c|c|c|c|}
\hline RL $1 . D$. & DOE I.D. & PH & COND & ALK & TH & AC & $A L$ & AS \\
\hline 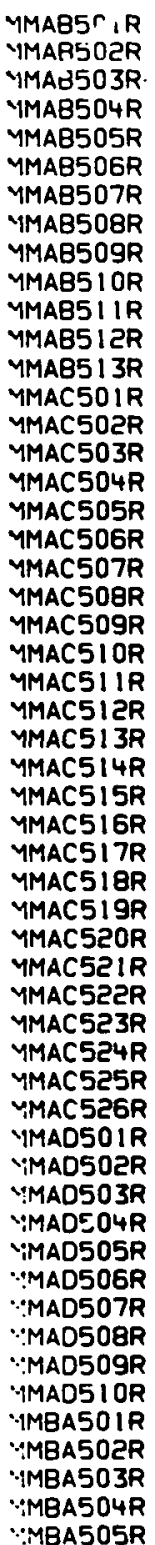 & 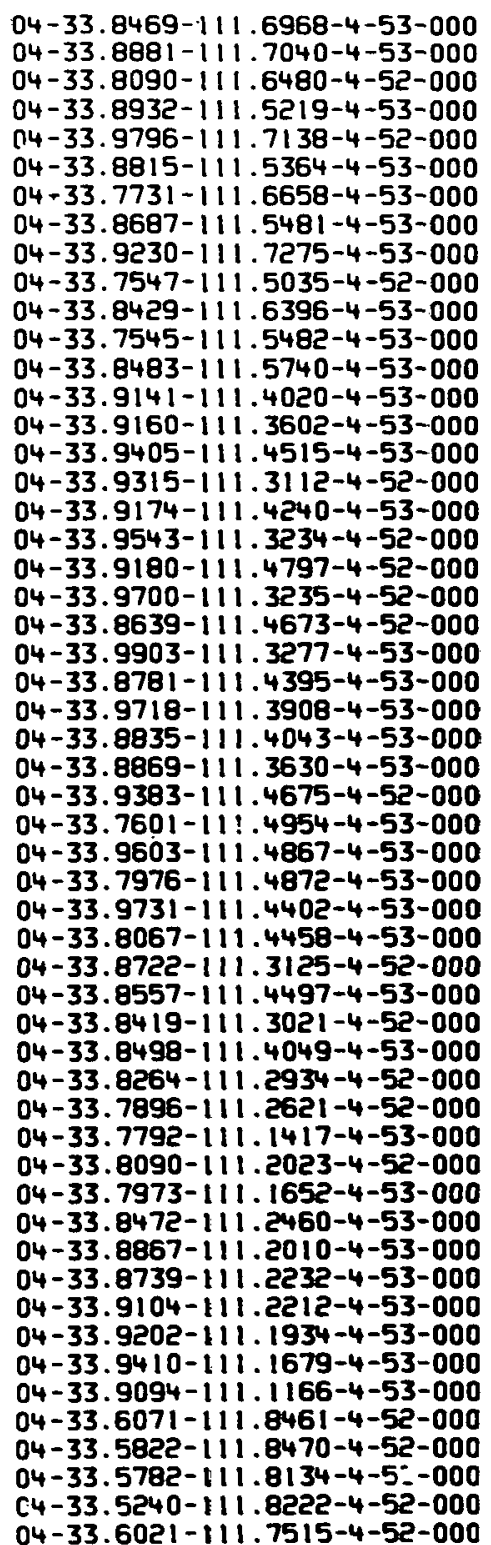 & $\begin{array}{l}7.4 \\
8.2 \\
7\end{array}$ & $\begin{array}{r}700 \\
550 \\
600 \\
364 \\
600 \\
392 \\
390 \\
840 \\
280 \\
270 \\
900 \\
600 \\
525 \\
60 \\
62 \\
510 \\
540 \\
100 \\
402 \\
455 \\
480 \\
580 \\
650 \\
340 \\
201 \\
270 \\
239 \\
510 \\
357 \\
352 \\
581 \\
218 \\
215 \\
320 \\
241 \\
990 \\
177 \\
990 \\
300 \\
600 \\
320 \\
800 \\
750 \\
480 \\
500 \\
650 \\
500 \\
270 \\
130 \\
500 \\
390 \\
480 \\
1600 \\
380\end{array}$ & $\begin{array}{l}7.10 \\
5.60 \\
4.60 \\
2.90 \\
4.10 \\
3.20 \\
3.90 \\
8.60 \\
1.90 \\
1.50 \\
5.90 \\
3.60 \\
3.70 \\
0.70 \\
0.46 \\
4.30 \\
3.40 \\
0.80 \\
3.70 \\
2.70 \\
5.10 \\
3.40 \\
6.70 \\
3.10 \\
2.00 \\
2.80 \\
2.30 \\
3.60 \\
1.90 \\
1.80 \\
4.70 \\
2.80 \\
0.90 \\
1.30 \\
4.00 \\
2.40 \\
1.50 \\
2.70 \\
1.80 \\
4.50 \\
3.20 \\
5.00 \\
4.30 \\
4.30 \\
4.00 \\
4.60 \\
4.80 \\
2.80 \\
0.60 \\
3.70 \\
3.30 \\
3.70 \\
2.60 \\
2.70\end{array}$ & $\begin{array}{r}-5 \\
-5 \\
-5 \\
20 \\
-5 \\
-5 \\
-5 \\
-5 \\
-5 \\
11 \\
-5 \\
-5 \\
-5 \\
-5 \\
-5 \\
9 \\
6 \\
-5 \\
11 \\
7 \\
-5 \\
-5 \\
-5 \\
-5 \\
-5 \\
-5 \\
-5 \\
9 \\
-5 \\
-5 \\
9 \\
-5 \\
9 \\
9 \\
6 \\
-5 \\
-5 \\
-5 \\
-5 \\
-5 \\
-5 \\
-5 \\
-5 \\
-5 \\
-5 \\
-5 \\
-5 \\
-5 \\
-5 \\
-5 \\
-5 \\
-5 \\
6\end{array}$ & $\begin{array}{r}-2 \\
-2 \\
-2 \\
3 \\
-2 \\
-2 \\
-2 \\
-2 \\
-2 \\
3 \\
-2 \\
-2 \\
-2 \\
-2 \\
-2 \\
-2 \\
2 \\
-2 \\
-2 \\
-2 \\
-2 \\
-2 \\
-2 \\
-2 \\
-2 \\
-2 \\
-2 \\
2 \\
-2 \\
-2 \\
2 \\
-2 \\
2 \\
2 \\
2 \\
-2 \\
-2 \\
-2 \\
-2 \\
2 \\
-2 \\
-2 \\
-2 \\
-2 \\
-2 \\
-2 \\
-2 \\
-2 \\
-2 \\
-2 \\
-2 \\
-2 \\
2 \\
2 \\
-2\end{array}$ & $\begin{array}{r}1051 \\
26 \\
21 \\
27 \\
87 \\
214 \\
929 \\
196 \\
39 \\
73 \\
33 \\
80 \\
20 \\
212 \\
164 \\
159 \\
31 \\
75 \\
15 \\
55 \\
42 \\
82 \\
43 \\
75 \\
65 \\
431 \\
77 \\
94 \\
36 \\
42 \\
33 \\
324 \\
38 \\
51 \\
28 \\
31 \\
114 \\
54 \\
172 \\
67 \\
111 \\
137 \\
117 \\
27 \\
132 \\
87 \\
784 \\
51 \\
149 \\
16 \\
30 \\
14 \\
44 \\
20\end{array}$ & $\begin{array}{r}1.6 \\
1.1 \\
8.7 \\
2.9 \\
6.7 \\
3.2 \\
2.3 \\
318.0 \\
4.5 \\
3.2 \\
1.1 \\
0.6 \\
8.7 \\
1.1 \\
0.8 \\
8.5 \\
4.1 \\
1.8 \\
1.2 \\
2.6 \\
8.2 \\
2.5 \\
16.1 \\
16.2 \\
4.5 \\
2.3 \\
-0.5 \\
0.9 \\
0.7 \\
-0.5 \\
8.0 \\
4.0 \\
0.6 \\
3.5 \\
3.0 \\
1.2 \\
-0.5 \\
0.6 \\
1.2 \\
-0.5 \\
-0.5 \\
2.1 \\
3.9 \\
2.6 \\
2.2 \\
2.3 \\
1.0 \\
-0.5 \\
-0.5 \\
5.5 \\
6.6\end{array}$ \\
\hline
\end{tabular}

B BA BE

CR

CU

$\begin{array}{rrr}110 & 10 & -1 \\ 44 & 4 & -1 \\ 154 & 5 & -1 \\ 37 & 10 & -1 \\ 188 & 25 & -1 \\ 41 & 19 & -1 \\ 43 & 39 & -1 \\ 349 & 221 & -1 \\ 30 & 12 & 2 \\ 25 & 14 & -1 \\ 226 & 48 & -1 \\ 89 & 64 & -1 \\ 611 & 6 & -1 \\ 22 & 13 & -1 \\ 15 & 24 & -1 \\ 38 & 84 & -1 \\ 56 & 250 & -1 \\ 20 & 18 & -1 \\ 62 & 108 & -1 \\ 39 & 151 & -1 \\ 49 & 92 & -1 \\ 40 & 49 & -1 \\ 153 & 203 & -1 \\ 29 & 128 & -1 \\ 24 & 25 & -1 \\ 31 & 36 & -1 \\ 34 & 38 & -1 \\ 49 & 41 & -1 \\ 43 & 30 & -1 \\ 38 & 13 & -1 \\ 128 & 6 & -1 \\ 29 & 96 & -1 \\ 26 & 18 & -1 \\ 69 & 58 & -1 \\ 30 & 49 & -1 \\ 311 & 152 & -1 \\ 22 & 10 & -1 \\ 285 & 135 & -1 \\ 52 & 16 & -1 \\ 76 & 23 & -1 \\ 89 & 50 & -1 \\ 120 & 46 & -1 \\ 98 & 87 & -1 \\ 48 & 101 & -1 \\ 66 & 53 & -1 \\ 52 & 102 & 1 \\ 53 & 98 & -1 \\ 31 & 33 & -1 \\ 22 & 43 & -1 \\ 112 & 67 & -1 \\ 104 & 35 & -1 \\ 116 & 62 & -1 \\ 184 & 34 & -1 \\ 129 & 15 & -1 \\ & & \end{array}$

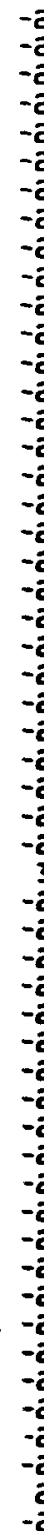


APPENDIX II TABULATION OF KEY FIELD MEASUREMENTS AND ANALYTICAL DATA--- GROUND WATER--EE LI MN MO NB NI F SC SE SR TI V Y ZN AND ZR CONCENTRATIONS IN PPB.
CA K MG NA AND SI CONCENTRATIONS IN PPM.

$\begin{array}{rrrr}\text { FE } & K & \text { L1 } & M G \\ 841 & 2.0 & 168 & 28.7 \\ -10 & 0.6 & 28 & 44.2 \\ -10 & 7.0 & 90 & 12.9 \\ -10 & 1.4 & 25 & 15.3 \\ 31 & 3.2 & 28 & 33.6 \\ 128 & 1.4 & 27 & 17.9 \\ 898 & 3.4 & 13 & 16.9 \\ 166 & 4.9 & 259 & 4.1 \\ -10 & 1.9 & 25 & 5.9 \\ 47 & 0.9 & 9 & 13.0 \\ -10 & 3.1 & 59 & 28.5 \\ -10 & 1.3 & 43 & 20.3 \\ -10 & 10.1 & 124 & 5.2 \\ 166 & 1.3 & 5 & 11.8 \\ 267 & 4.0 & -2 & 1.6 \\ 201 & 1.3 & 78 & 25.1 \\ -10 & 5.5 & 26 & 24.2 \\ -10 & 0.4 & 12 & 3.3 \\ -10 & 1.8 & 23 & 14.3 \\ -10 & 1.9 & 31 & 15.9 \\ -10 & 1.7 & 25 & 26.7 \\ 24 & 2.0 & 15 & 29.0 \\ -10 & 4.8 & 85 & 21.7 \\ 42 & 1.7 & 21 & 16.0 \\ -10 & 1.0 & 5 & 6.4 \\ 388 & 2.0 & 24 & 12.0 \\ -10 & 0.6 & -2 & 15.8 \\ 100 & 1.4 & 10 & 17.9 \\ -10 & 1.5 & 48 & 9.2 \\ -10 & 0.9 & 10 & 35.9 \\ 77 & 3.3 & 139 & 1.8 \\ 614 & 1.5 & 11 & 6.8 \\ -10 & 1.1 & 10 & 5.0 \\ 72 & 1.0 & 18 & 16.2 \\ 12 & 1.7 & 8 & 16.8 \\ 12 & 2.3 & 34 & 27.2 \\ 32 & 2.5 & 11 & 6.6 \\ 34 & 2.0 & 39 & 31.3 \\ 122 & 0.7 & 18 & 9.4 \\ 30 & 8.0 & 18 & 55.7 \\ 41.4 & 3.0 & 7 & 27.3 \\ 675 & 14.0 & 32 & 73.2 \\ 391 & 2.9 & 54 & 34.0 \\ 38 & 3.5 & 14 & 31.5 \\ 350 & 4.4 & 25 & 34.7 \\ 28 & 1.8 & 46 & 42.2 \\ 739 & 2.4 & 30 & 39.2 \\ 141 & 3.4 & 3 & 25.5 \\ 124 & 2.1 & -2 & 6.7 \\ -10 & 2.5 & 33 & 13.2 \\ -10 & 3.6 & 79 & 13.4 \\ -10 & 2.6 & 30 & 9.9 \\ 22 & 6.7 & 124 & 41.2 \\ -10 & 4.0 & 47 & 14.3\end{array}$

$\begin{array}{rr}M N & M \\ 45 & \\ 5 & -4 \\ -2 & -4 \\ 4 & \\ 5 & \\ 22 & -4 \\ 24 & -4 \\ 773 & -4 \\ 3 & -4 \\ 4 & \\ 3 & 58 \\ 8 & -4 \\ -2 & 13 \\ 5 & -4 \\ 43 & -4 \\ 30 & \\ 9 & \\ 4 & -4 \\ 20 & -4 \\ 8 & -4 \\ 6 & -4 \\ 5 & -4 \\ 3 & \\ 7 & -4 \\ -2 & -4 \\ 138 & \\ 4 & \\ 18 & -4 \\ 3 & -4 \\ 6 & -4 \\ 6 & 25 \\ 52 & -4 \\ 4 & -4 \\ 743 & \\ 14 & \\ 24 & 4 \\ 4 & \\ 21 & \\ 11 & \\ 26 & \\ 16 & \\ 23 & \\ 15 & 131 \\ 15 & 10 \\ 16 & 28 \\ 9 & -4 \\ 3 & -2 \\ 19 & \\ 4 & \end{array}$

1
7
-4
-4
5
5
-4
-4
-4
-4
7
58
-4
13
-4
-4
4
4
-4
-4
-4
-4
-4
4
-4
-4
5
6
-4
-4
-4
25
-4
-4
-4
-4
5
-4
-4
-4
-4
-4
5
-4
-4
-4
11
-4
-4
-4
-4
12
-4
4
-4

SE

SI SR

$82 \quad \begin{array}{llll}1.0 & 0.3 & 38.2\end{array}$

88.6
30.8

95.9

35.9

33.2

14.0

197.8

MMABSO9R

MMAB5 IIR

MMAB513R

YMAC5O $1 R$

YMAC504R

MMAC505R

MMAC5O6R

YMAC508R

MMAC509R

MMAC5!IR

MMAC5IIR

MMAC513R

MMAC514R

MMAC5I $6 R$

MMAC5I $8 R$

MMAC519R

MMAC52OR

MMAC522R

MMAC523R

MMAC524R

MMAC525R

MMAO5OIR

MMADSOZR

MMAD503R

MMAD504R

MMAD506R

MMAD507R

MMAD50BR

MMAD509R

MMBA5OIR

MMBA502R

MMBA503R

MMBA505R

16.7

110.4
68.3

138.7

11.1

40.4

41.5
8.3

25.0

25. 0

टर. 4

22. 1

13.3

13.3

12.8

29.8
16.7

144.7

15.0
10.9

11.7

25.6

48.1

55.7

21.5

18.1

39.4

67.9
27.0

29.6

56.4

12.6

4.5
56.8

56.8
55.5

63.1
163.2

\begin{tabular}{rr}
$N B$ & $N 1$ \\
-4 & -4 \\
-4 & 7 \\
-4 & 6 \\
7 & 5 \\
-4 & 6 \\
-4 & -4 \\
-4 & -4 \\
-4 & 8 \\
-4 & -4 \\
6 & -4 \\
-4 & -4 \\
-4 & 7 \\
6 & -4 \\
-4 & 5 \\
-4 & -6 \\
-4 & 9 \\
6 & 6 \\
6 & -4 \\
-4 & -4 \\
-4 & 7 \\
-4 & 4 \\
-4 & -4 \\
-4 & -4 \\
-4 & 5 \\
-4 & 6 \\
10 & -4 \\
-4 & -4 \\
-4 & 5 \\
-4 & -4 \\
-4 & 5 \\
-4 & -4 \\
6 & -4 \\
-4 & -4 \\
-4 & 9 \\
5 & 6 \\
-4 & -4 \\
-4 & -4 \\
-4 & 8 \\
-4 & 4 \\
6 & -4 \\
-4 & -4 \\
-4 & 7 \\
-4 & 5 \\
-4 & 7 \\
-4 & 6 \\
-4 & -4 \\
-4 & 7 \\
-4 & 5 \\
-4 & -4 \\
-4 & -4 \\
4 & -4 \\
-4 & 6 \\
-4 & 8 \\
-4 & -4 \\
\hline
\end{tabular}

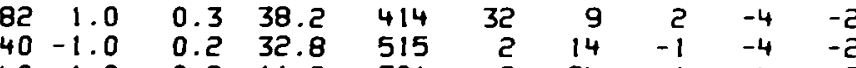

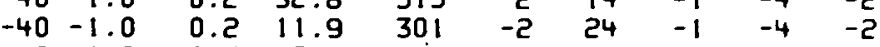

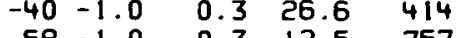

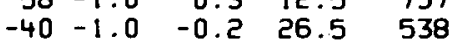

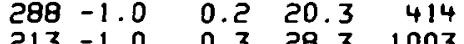

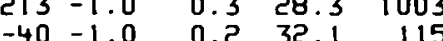

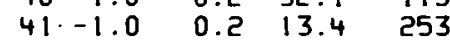

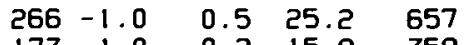

$\begin{array}{lllll}173 & -1.0 & 0.2 & 15.8 & 358\end{array}$

$\begin{array}{lllll}-40 & -1.0 & 0.2 & 31.0 & 156\end{array}$

$\begin{array}{lll}-1.0 & 0.2 & 9.8\end{array}$

$\begin{array}{lllll}252 & -1.0 & 0.5 & 3.4 & 51\end{array}$

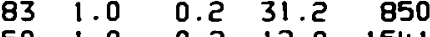

$\begin{array}{rrrrr}50 & -1.0 & -0.2 & 12.0 & 1541 \\ 50 & -1.0 & 0.3 & 12.3 & 158\end{array}$

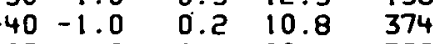

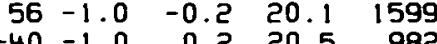

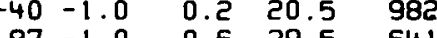

$\begin{array}{rrrrr}87 & -1.0 & 0.6 & 29.5 & 641\end{array}$

$\begin{array}{rrrr}7.9-1.0 & 0.9 & 13.7 & 1311 \\ -40 & 0.2 & 21.4 & 316\end{array}$

$\begin{array}{lllll}-40 & -1.0 & 0.3 & 15.0 & 137\end{array}$

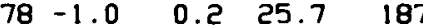

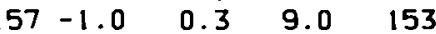

$\begin{array}{lllll}-40 & -1.0 & 0.4 & 9.4 & 345\end{array}$

$\begin{array}{rrrrr}88 & -1.0 & 0.2 & 17.7 & 229 \\ -40 & -1.0 & -0.2 & 11.2 & 220\end{array}$

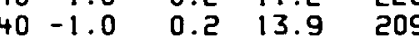

$\begin{array}{lllll}110 & -1.0 & -0.2 & 17.2 & 175\end{array}$

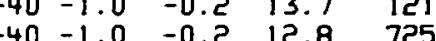

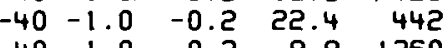

$\begin{array}{lllll}-40 & -1.0 & -0.2 & 8.8 & 1260\end{array}$

$\begin{array}{lllll}-40 & -1.0 & -0.2 & 17.9 & 100\end{array}$

$53-1.0 .0 .2 \quad 9.7-1506$

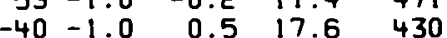

$\begin{array}{lllll}-40 & -1.0 & -0.2 & 12.7 & 242\end{array}$

$\begin{array}{rrrrr}41 & -1.0 & 0.2 & 18.6 & 445 \\ -40 & -1.0 & -0.2 & 21.7 & 323\end{array}$

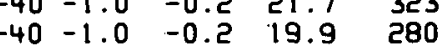

$\begin{array}{lllll}-40 & -1.0 & -0.2 & 19.5 & 393\end{array}$

$\begin{array}{lllll}-40 & 1.0 & -0.2 & 17.6 & 321\end{array}$

$136-1.0-0.519 .4236$

$\begin{array}{llll}59-1.0 & -0.2 & 10.8 & 74\end{array}$

$\begin{array}{lllll}-40 & -1.0 & 0.3 & 18.8 & 473\end{array}$

$\begin{array}{lllll}-40 & -1.0 & -0.2 & 15.4 & 722\end{array}$

$-40-1.0-0.2 \cdot 20.3 \quad 391$

$\begin{array}{rrrrr}-40 & -1.0 & -0.2 & 15.7 & 1304 \\ -40 & -1.0 & -0.2 & 20.7 & 772\end{array}$

47.1 

ELEMENTAL CONCENTRATIJNS IN PPB. ALK IN MEQ/L. COND IN UM/CM.

SRL $1 . D$.

DOE I.D.

PH COND ALK

YMBA5 JR $04-33.6026-111.8840-4-52-000$ MMBASOTR $04-33.7480-111.9903-4-52-0007.7$ YMBA50BR 04-33.5795-111.9727-4-52-000 $84-33.5795-111.9727-4-52-0008.1 \quad 4003.70$ MMBA510R $04-33.6550-111.9255-4-52-0007.9 \quad 4404.30$ YMBASIIR 04-33.7270-111.9300-4-52-000 7.4 5504.30 YMBA5I2R $04-33.7300-111.8782-4-52-0007.723001 .50$ YMBA5I3R 04-33.6977-111.8943-4-52-000 7.5 10502.20 4MBA5I4R 04-33.6920-111. 9245-4-52-000 7.7 6004.00 YMBA5I5R $04-33.6516-111.9682-4-52-0007.9$ 400 4.00 MMBASISR $04-33.6516-111.9682-4-52-0007.914004 .00$

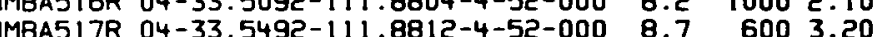
MMBA5IBR 04-33.5962-111.9521-4-52-000 $8.7 \quad 3202.60$ MMBA5ISR $04-33.5426-111.9914-4-52-000$ 8.0 3503.10 MMBA52OR 04-33.5537-111.9258-4-52-000 $8.3 \quad 4603.30$ MMBA52IR $04-33.7322-111.8456-4-52-000 \quad 7.2 \quad 2801.30$ MMBA522R $04-33.7349-111.7879-4-52-000 \quad 7.8 \quad 5206.10$ MMBA523R $0^{1} 4-33.6717-111.8216-4-53-000 \quad 7.8 \quad 7005.20$ MMBB50IR $04-33.5130-111.6880-4-52-000 \quad 7.4 \quad 19508.30$ 4MBB502R $04-33.5523-111.6064-4-52-600 \quad 7.5 \quad 6252.30$ YMBB503R $04-33.6006-111.7145-4-52-000 \quad 7.6 \quad 4603.50$ 4MBB504R 04-33.6037-111.6915-4-52-000 B.2 3302.60 MMB日506R 04-33.7284-111.6551-4-52-000 8.1 5001.80 MMBB507R 04-33.7466-111.5975-4-53-000 7.3 MMBB508R 04-33.7370-11. 5760-4-53-000 7.7 4MB $04-33.73708-111.5145-4-53-000$ 8.1 MBBSOPR $04-33.7108-111.6145-4-53-000$ 8.1 YMBDSOIR 04-33.6034-111.0059-4-52-000 8.8 YMBO5O2R $04-33.5877-111.0631-4-52-0007.9$ MMBD503R 04-33.6508-111.0471-4-53-000 7.6 MMBD504R 04-33.6461-111.1110-4-52-000 7.2 MMBD505R 04-33.6715-111. 1332-4-52-000 7.7 YMBD506R 04-33.6236-111.0021-4-52-000 7.4 MMBDS07R $04-33.6103-111.0905-4-52-0007.6$ MBD508R 04-33.5950-111.2208-4-52-000 7.6 MMBD509R 04-33.6597-111.2202-4-52-000 7.9 YMBDSIOR 04-33.7479-111.0394-4-52-000 B. YMBO5I2R 04-33.7327-111.1092-4-52-000 7.8 YMBD5 I 3R 04-33.7295-111.1312-4-53-000 8.1 YMBD5 IUR 04-33.5482-111.0662-4-53-000 7.7 YMBD515R 04-33.5276-111.0826-4-53-000 7.7 YMBE50IR 04-33.5182-110.8624-4-53-000 7.3 4MBED $04-33.5440-110.7902-4-53-000 \cdot 7.3$ YMBES03R 04-33.5513-110.8338-4-52-000 9.0 YMBESOBR $04-33.5513-110.8328-4-52-000$ 8. YMBESO4R $04-33.5595-110.8844-4-52-0005.9$ MBESOSR 04-33.5893-110.8505-4-52-000 7.9 MMBESOGR O4-33.5930-110.8776-4-53-000 7.5 MMBESOTR 04-33.5913-110.7858-4-53-000 7.7 MMBE5OBR 04-33.6095-110.7972-4-53-000 7.1 MMBE5O9R 04-33.7126-110.9511-4-53-000 7.7 MMBESIOR 04-33.6566-110.9245-4-52-000 7.4 -1MBESIIR 04-33.6894-110.8716-4-5:-000 8.4 AMBESILR $04-33.6940-110.8123-4-52-0008.0$ 8504.60 3702.60 4002.50 5104.00 4873.60 7605.80 4142.70 18803.90 6904.20 3333.20 4173.50 4402.90 4803.40 4504.20 4204.80 3903.10 7004.50 17904.50 17900.30 402 2.60 4002.60 7503.10 7505.30 4974.10 6105.30 3512.50 5802.70

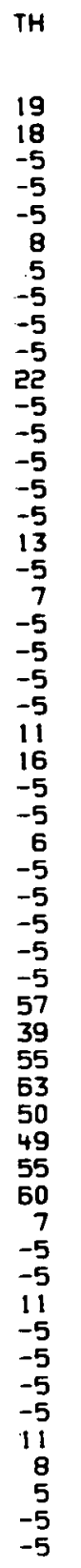

AL

B

CA

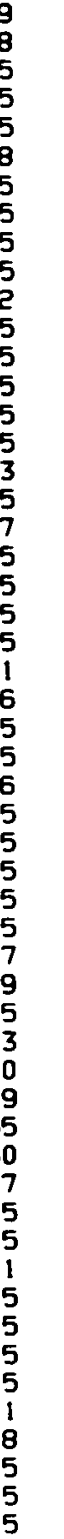

30
185
28
18
42
29
19
19
-10
15
17
-10
42
36
101
23
25
-10
30
20
11
20
22
167
118
1014
110
47
36
61
26
53
289
28
33
44
53
51
64
69
52
31
94
-10
15
35
71
805
42
58
31
31
22
21
19
23

6.4 5.0

4.5

8.8

4.2

14.6

3.5

3.5

4.6
10.5

6.1

4.7

10.0

0.9

6.3

2. 7

5.1

10.9

6.0

1.0
2.7

1.6
11.2

2.1

-0.5
0.6

35.9

1.8
-0.5

2. 8

4.1

1.5

1.3

3.7

3.2

3.2

4.4

5.8

3.5

6.9

2.9
0.9

0.9
1.1
109
70
82
91
93
72
191
114
88
70
123
130
100
66
132
49
135
176
497
114
117
166
220
140
88
43
621
64
59
75
29
464
83
106
19
35
56
51
58
43
31
38
62
79
79
48
45
58
136
73
72
100
25
31

$\begin{array}{rrrrr}25 & 38 & -2 & 40 & 3 \\ 33 & 43 & -2 & 12 & 3 \\ 23 & -30 & -2 & 14 & 4 \\ 17 & -30 & -2 & 112 & 3 \\ 13 & -30 & -2 & 37 & -2 \\ 43 & -30 & -2 & 5 & 36 \\ 283 & -30 & -2 & -4 & 11 \\ 60 & -30 & -2 & -4 & 2 \\ 53 & -30 & -2 & 4 & 2 \\ 15 & -30 & -2 & 71 & 2 \\ 34 & 44 & 2 & 18 & 23 \\ 5 & -30 & -2 & 29 & -2 \\ 4 & -30 & -2 & 158 & -2 \\ 25 & -30 & -2 & 28 & 3 \\ 15 & -30 & -2 & 72 & -2 \\ 37 & -30 & 3 & -4 & 5 \\ 90 & -30 & 3 & -4 & 7 \\ 98 & -30 & -2 & -4 & 4 \\ 112 & -30 & -2 & -4 & 81 \\ 30 & -30 & -2 & -4 & 4 \\ 35 & -30 & -2 & -4 & 5 \\ 25 & -30 & -2 & 12 & 38 \\ 22 & -30 & -2 & 21 & -2 \\ 68 & -30 & -2 & 5 & 14 \\ 37 & 31 & 4 & 9 & 26 \\ 63 & -30 & -2 & -4 & 12 \\ 3 & -30 & -2 & -4 & 3 \\ 62 & -30 & -2 & -4 & 7 \\ 61 & -30 & -2 & -4 & 4 \\ 6 & -30 & -2 & -4 & 10 \\ 64 & -30 & -2 & -4 & 13 \\ 35 & -30 & -2 & -4 & 10 \\ 53 & -30 & -2 & -4 & 3 \\ 73 & -30 & -2 & -4 & 13 \\ 49 & -30 & -2 & -4 & 3 \\ 63 & 30 & 6 & -4 & 5 \\ 63 & 37 & 4 & 6 & 5 \\ 53 & -30 & 6 & -4 & 15 \\ 37 & 44 & 7 & 15 & 25 \\ 59 & -30 & 4 & -4 & 10 \\ 62 & 34 & 6 & 5 & 2 \\ 58 & -30 & 2 & -4 & 8 \\ 81 & -30 & -2 & -4 & 11 \\ 82 & -30 & -2 & -4 & 21 \\ 347 & -30 & 18 & -4 & 576 \\ 71 & -30 & -2 & -4 & 34 \\ 60 & -30 & -2 & -4 & 28 \\ 79 & -30 & -2 & -4 & 15 \\ 89 & -30 & -2 & -4 & 10 \\ 64 & -30 & -2 & 4 & 2 \\ 79 & -30 & -2 & 4 & 6 \\ 53 & -30 & -2 & -4 & 6 \\ 37 & -30 & -2 & -4 & 16 \\ 71 & -30 & -2 & -4 & 7\end{array}$


APPENDIX 11 TABULATION OF KEY FIELD MEASUREMENTS AND ANALYTICAL DATA-- - GROUND WATER--

MESA SHEET

IX 11 TABULATION OF KEY FIELD MEASUREMENTS AND AN

CA K MG NA AND SI CONCENTRATIONS IN PPM.

$\begin{array}{lrrrr}\text { SRL I.D. } & \text { FE } & K & L 1 & \text { MG } \\ \text { MMBA506R } & -10 & 3.7 & 50 & 16.9 \\ \text { MMBA507R } & 345 & 2.2 & 24 & 20.5 \\ \text { MMBA50BR } & -10 & 2.0 & 24 & 18.7 \\ \text { MMBA509R } & -10 & 2.3 & 25 & 12.7 \\ \text { MMBA510R } & -10 & 3.2 & 47 & 12.9 \\ \text { MMBA511R } & 44 & 2.4 & 25 & 14.5 \\ \text { MMBA512R } & -10 & 9.9 & 193 & 57.4 \\ \text { MMBA513R } & -10 & 4.5 & 152 & 16.3 \\ \text { MMBA514R } & -10 & 2.5 & 31 & 16.6 \\ \text { MMBA515R } & -10 & 3.0 & 33 & 25.6 \\ \text { MMBA516R } & 154 & 4.3 & 125 & 23.4 \\ \text { MMBA517R } & -10 & 1.4 & 41 & 2.4 \\ \text { MMBA51BR } & -10 & 1.1 & 8 & 2.5 \\ \text { MMBA519R } & 26 & 2.7 & 22 & 19.1 \\ \text { MMBA520R } & -10 & 2.3 & 24 & 13.7 \\ \text { MMBA521R } & -10 & 1.6 & 23 & 9.6 \\ \text { MMBA522R } & -10 & 2.9 & 33 & 11.3 \\ \text { MMBA523R } & -10 & 4.0 & 48 & 24.9 \\ \text { MMBB501R } & -10 & 2.7 & 68 & 42.5 \\ \text { MMBB502R } & 589 & 3.4 & 70 & 8.3 \\ \text { YMBB503R } & -10 & 3.5 & 36 & 15.8 \\ \text { MMBB504R } & -10 & 3.9 & 41 & 16.2 \\ \text { MMBB506R } & -10 & 2.0 & 92 & 22.9 \\ \text { MMBB507R } & 71 & 1.9 & 61 & 34.7 \\ \text { MMBB508R } & 936 & 3.8 & 38 & 13.2 \\ \text { MMBB509R } & 65 & 2.2 & 19 & 19.7 \\ \text { MMBD501R } & 53 & 1.4 & 98 & 0.9 \\ \text { MMBD502R } & -10 & 1.9 & 42 & 18.1 \\ \text { MMBD503R } & -10 & 2.4 & 38 & 15.9 \\ \text { MMBD504R } & 606 & 2.8 & 4 & 4.7 \\ \text { MMBD505R } & -10 & 1.6 & 11 & 18.5 \\ \text { MMBD506R } & 129 & 9.8 & 664 & 9.2 \\ \text { MMBD507R } & -10 & 2.5 & 55 & 35.8 \\ \text { MMBD508R } & -10 & 2.6 & 76 & 30.4 \\ \text { MMBD509R } & -10 & 3.0 & 3 & 20.3 \\ \text { MMBD510R } & -10 & 2.3 & 9 & 21.5 \\ \text { MMBD511R } & -10 & 2.8 & 16 & 21.0 \\ \text { MMBD512R } & -10 & 2.5 & 29 & 28.6 \\ \text { MMBD513R } & -10 & 3.1 & 23 & 36.4 \\ \text { MMBD514R } & -10 & 1.3 & 110 & 15.8 \\ \text { MMBD515R } & -10 & 1.4 & 43 & 16.9 \\ \text { MMBE501R } & -10 & 2.9 & 32 & 25.8 \\ \text { MMBE502R } & 48 & 1.9 & 20 & 46.7 \\ \text { MMBE503R } & 66 & 3.4 & 70 & 46.8 \\ \text { MMBE504R } & 325 & 5.5 & 71 & 82.0 \\ \text { MMBE505R } & 61 & 2.6 & 54 & 17.6 \\ \text { MMBE506R } & 19 & 2.4 & 44 & 10.0 \\ \text { MMBE507R } & -10 & 1.9 & 25 & 30.0 \\ \text { MMBE508R } & -10 & 1.1 & 102 & 34.8 \\ \text { MMBE509R } & -10 & 4.6 & 15 & 49.0 \\ \text { MMBE510R } & -10 & 2.7 & 33 & 19.8 \\ \text { MMBE511R } & -10 & 6.1 & 57 & 24.0 \\ \text { MMBE512R } & -10 & 1.3 & 6 & 14.9 \\ \text { MMBE513R } & -10 & 2.2 & 16 & 21.3\end{array}$

\begin{tabular}{|c|c|c|c|c|}
\hline MN & MO & NA & NB & NI \\
\hline 2 & -4 & 54.3 & -4 & 10 \\
\hline $1 \overline{2}$ & 4 & 29.5 & -4 & 5 \\
\hline 2 & 7 & 35.1 & -4 & 7 \\
\hline$-\overline{2}$ & 4 & 47.3 & -4 & 7 \\
\hline 2 & 4 & 56.7 & -4 & -4 \\
\hline 7 & -4 & 48.2 & -4. & -4 \\
\hline 8 & -4 & 183.2 & -4 & -4 \\
\hline 2 & -4 & 101.3 & -4 & -4 \\
\hline 2 & -4 & 45.7 & -4 & -4 \\
\hline 2 & -4 & 32.4 & -4 & -4 \\
\hline-2 & 4 & 137.0 & 8 & -4 \\
\hline$-\overline{2}$ & -4 & 110.9 & 5 & -4 \\
\hline 3 & -4 & 62.4 & -4 & -4 \\
\hline 3 & -4 & 28.5 & -4 & -4 \\
\hline-2 & -4 & 63.2 & -4 & -4 \\
\hline 11 & -4 & 21.0 & -4 & -4 \\
\hline 3 & -4 & 49.0 & -4 & 5 \\
\hline 11 & 9 & 59.4 & -4 & -4 \\
\hline 7 & -4 & 286.7 & -4 & 6 \\
\hline 13 & -4 & 122.5 & -4 & 9 \\
\hline 20 & -4 & 32.7 & -4 & $\boldsymbol{\theta}$ \\
\hline ? & 4 & 30.6 & -4 & 15 \\
\hline-2 & 4 & 65.7 & 6 & 4 \\
\hline 7 & -4 & 115.6 & 4 & 6 \\
\hline 260 & 9 & 45.2 & 5 & 10 \\
\hline $\begin{array}{l}12 \\
-2\end{array}$ & 4 & 13.2 & -4 & 7 \\
\hline-2 & -4 & 157.6 & 5 & 8 \\
\hline 3 & -4 & 30.3 & 6 & -4 \\
\hline $\begin{array}{l}12 \\
17\end{array}$ & -4 & 27.4 & -4 & 4 \\
\hline 17 & -4 & 188.8 & -4 & 6 \\
\hline 12 & -4 & 17.5 & -4 & 5 \\
\hline 11 & 9 & 260.7 & -4 & -4 \\
\hline 7 & -4 & 42.5 & -4 & 6 \\
\hline 3 & 22 & 95.4 & -4 & 4 \\
\hline 2 & -4 & 22.5. & -4 & -4 \\
\hline 5 & -4 & 20.7 & 15 & 7 \\
\hline 4 & -4 & 26.9 & .12 & 4 \\
\hline 2 & 7 & 30.7 & 7 & -4 \\
\hline 5 & 7 & 42.2 & 16 & 12 \\
\hline $\begin{array}{l}4 \\
3\end{array}$ & $\begin{array}{l}6 \\
7\end{array}$ & $\begin{array}{l}42.5 \\
25.0\end{array}$ & $\begin{array}{r}5 \\
12\end{array}$ & $\begin{array}{l}12 \\
-4\end{array}$ \\
\hline 3 & -4 & 23.0 & 8 & -4 \\
\hline 4 & -4 & 42.7 & -4 & -4 \\
\hline 12 & -4 & 46.0 & -4 & -4 \\
\hline 4392 & -4 & 45.7 & -4 & 96 \\
\hline 230 & -4 & 29.4 & -4 & $\mathbf{9}$ \\
\hline 19 & -4 & 26.2 & 5 & 5 \\
\hline 5 & -4 & 29.4 & 4 & 6 \\
\hline 4 & -4 & 61.7 & -4 & 10 \\
\hline $\begin{array}{l}5 \\
3\end{array}$ & $\begin{array}{l}-4 \\
-4\end{array}$ & $\begin{array}{r}34.8 \\
8.4\end{array}$ & $\begin{array}{l}4 \\
8\end{array}$ & 5 \\
\hline 3 & -4 & 64.6 & -4 & -4 \\
\hline 3 & -4 & 9.5 & -4 & -4 \\
\hline 3 & 4 & 19.8 & -4 & -4 \\
\hline
\end{tabular}

$\begin{array}{rrrrr}\text { P } & \text { SC } & \text { SE } & \text { S1 } & \text { SR } \\ -40 & -1.0 & -0.2 & 15.8 & 943 \\ -40 & -1.0 & -0.2 & 21.0 & 535 \\ -40 & -1.0 & -0.2 & 14.6 & 591 \\ -40 & -1.0 & -0.2 & 12.9 & 484 \\ -40 & -1.0 & 0.3 & 9.5 & 496 \\ -40 & -1.0 & 0.2 & 16.7 & 391 \\ -40 & 1.0 & 0.3 & 5.8 & 7713 \\ -40 & -1.0 & -0.2 & 14.6 & 918 \\ -40 & -1.0 & 0.4 & 19.2 & 419 \\ -40 & -1.0 & 0.3 & 10.2 & 491 \\ -40 & -1.0 & 0.2 & 9.7 & 435 \\ -40 & -1.0 & -0.2 & 7.6 & 91 \\ -40 & -1.0 & 0.3 & 6.6 & 147 \\ -40 & -1.0 & -0.2 & 14.5 & 404 \\ -40 & -1.0 & 0.4 & 9.9 & 513 \\ 44 & -1.0 & 0.2 & 9.1 & 225 \\ -40 & 1.0 & 0.2 & 14.5 & 332 \\ -40 & -1.0 & 0.5 & 15.7 & 559 \\ 66 & 1.0 & 0.5 & 24.7 & 1081 \\ 82 & -1.0 & -0.2 & 10.0 & 237 \\ -40 & -1.0 & -0.2 & 24.5 & 562 \\ -40 & -1.0 & -0.2 & 18.1 & 603 \\ -40 & -1.0 & -0.2 & 17.0 & 155 \\ 60 & -1.0 & -0.2 & 32.4 & 668 \\ 319 & -1.0 & 0.2 & 18.2 & 226 \\ -40 & -1.0 & -0.2 & 24.0 & 521 \\ -40 & -1.0 & -0.2 & 21.2 & 45 \\ -40 & -1.0 & -0.2 & 18.5 & 227 \\ 55 & -1.0 & -0.2 & 20.6 & 272 \\ -40 & -1.0 & -0.2 & 12.7 & 21 \\ -40 & -1.0 & -0.2 & 11.4 & 285 \\ -40 & -1.0 & -0.2 & 15.6 & 456 \\ -40 & -1.0 & -0.2 & 15.8 & 191 \\ 72 & -1.0 & 0.3 & 15.0 & 1007 \\ 71 & -1.0 & 0.3 & 11.7 & 183 \\ -40 & -1.0 & 0.3 & 15.8 & 179 \\ 42 & 1.0 & 0.3 & 15.6 & 235 \\ 52 & -1.0 & 0.5 & 16.7 & 385 \\ 46 & 1.0 & 0.3 & 10.8 & 234 \\ 59 & -1.0 & -0.2 & 28.0 & 214 \\ -40 & 1.0 & 0.3 & 17.6 & 135 \\ -40 & -1.0 & 0.3 & 14.2 & 532 \\ -40 & -1.0 & 0.5 & 17.8 & 361 \\ -40 & -1.0 & 0.5 & 23.5 & 654 \\ 254 & 1.0 & 0.3 & 19.1 & 1459 \\ -40 & -1.0 & 0.2 & 17.0 & 395 \\ -40 & -1.0 & 0.4 & 21.4 & 230 \\ 55 & -1.0 & 0.3 & 15.2 & 234 \\ -40 & -1.0 & 0.3 & 24.3 & 307 \\ -40 & 1.0 & 0.3 & 20.3 & 233 \\ -40 & -1.0 & 0.3 & 23.6 & 463 \\ -40 & -1.0 & 0.3 & 15.5 & 831 \\ -40 & -1.0 & 0.3 & 7.8 & 141 \\ -40 & -1.0 & 0.5 & 12.0 & 323 \\ & & & & \end{array}$

\begin{tabular}{|c|c|c|c|c|}
\hline 11 & $v$ & $Y$ & $Z N$ & $Z R$ \\
\hline-2 & 21 & 1 & 97 & \\
\hline 0 & 30 & 1 & 200 & \\
\hline-2 & 25 & $-i$ & 79 & -2 \\
\hline & 21 & -1 & 4 & $-a$ \\
\hline 3 & 18 & -1 & 112 & -2 \\
\hline 3 & 11 & $i$ & -4 & \\
\hline 8 & -4 & -1 & 708 & $-i$ \\
\hline 3 & -4 & -1 & -4 & $-z$ \\
\hline 3 & 8 & -1 & -4 & $-a$ \\
\hline 2 & 25 & -1 & -4 & $-z$ \\
\hline 3 & 18 & $i$ & -4 & \\
\hline 3 & 42 & -1 & -4 & -2 \\
\hline$-\overline{2}$ & 35 & -1 & 130 & $-a$ \\
\hline $\begin{array}{r}5 \\
-2\end{array}$ & $\begin{array}{l}23 \\
47\end{array}$ & $\begin{array}{l}-1 \\
-1\end{array}$ & $\begin{array}{l}-4 \\
-4\end{array}$ & $-a$ \\
\hline 2 & -4 & -1 & 727 & $-c$ \\
\hline 4 & 5 & $i$ & 2765 & \\
\hline 3 & -4 & -1 & -4 & \\
\hline 4 & 12 & 1 & 25 & - \\
\hline 3 & -4 & -1 & 124 & \\
\hline 2 & 16 & -1 & 33 & \\
\hline 2 & 22 & $\begin{array}{l}-1 \\
-1\end{array}$ & $\begin{array}{l}21 \\
-4\end{array}$ & 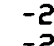 \\
\hline $\begin{array}{r}-c \\
6\end{array}$ & $\begin{array}{l}14 \\
14\end{array}$ & 1 & $\begin{array}{l}-4 \\
11\end{array}$ & $C$ \\
\hline 14 & 11 & 3 & -4 & 2 \\
\hline 4 & 13 & -1 & -4 & $-a$ \\
\hline 2 & 80 & -1 & 20 & -8 \\
\hline $\begin{array}{l}3 \\
3\end{array}$ & $\begin{array}{r}13 \\
6\end{array}$ & $\begin{array}{l}-1 \\
-1\end{array}$ & $\begin{array}{c}94 \\
-4\end{array}$ & $-\hat{c}$ \\
\hline-2 & -4 & $-i$ & 16 & $-c$ \\
\hline 2 & 8 & $-i$ & 466 & - \\
\hline 7 & 6 & -1 & 24 & $-i$ \\
\hline 3 & 4 & -1 & -4 & -2 \\
\hline 3 & 11 & 1 & 157 & \\
\hline 2 & 14 & -1 & -4 & \\
\hline $\begin{array}{l}3 \\
4\end{array}$ & $\begin{array}{l}15 \\
11\end{array}$ & $\begin{array}{l}1 \\
2\end{array}$ & $\begin{array}{r}90 \\
9\end{array}$ & \\
\hline 4 & 38 & 1 & 163 & \\
\hline 5 & 28 & 2 & 8 & \\
\hline 4 & 9 & 1 & -4 & \\
\hline 4 & 9 & 1 & -4 & - \\
\hline 2 & 15 & 1 & $\begin{array}{r}23 \\
781\end{array}$ & \\
\hline $\begin{array}{l}\mathrm{C} \\
2\end{array}$ & $\begin{array}{l}8 \\
7\end{array}$ & $\begin{array}{l}-1 \\
-1\end{array}$ & 443 & \\
\hline 9 & -4 & 11 & 980 & - \\
\hline 3 & -4 & -1 & 284 & \\
\hline 3 & 8 & -1 & 97 & \\
\hline ? & $\begin{array}{r}-4 \\
4\end{array}$ & $\begin{array}{l}-1 \\
-1\end{array}$ & 300 & \\
\hline 3 & 23 & $i$ & 208 & \\
\hline ? & 11 & $\begin{array}{r}1 \\
-1\end{array}$ & $\begin{array}{l}560 \\
224\end{array}$ & \\
\hline 2 & -4 & -1 & 66 & \\
\hline 3 & -4 & -1 & & \\
\hline
\end{tabular}


ERL I.D.

DOE I.D.

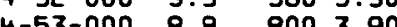
$M B-516 R \quad 04-33.6333-113.9376-4-52-000 \quad 7.423204 .00$ MMBE517R $04-33.7351-113.9822-4-53-0007.7 \quad 7204.20$ YMBE5IBR $04-33.7252-113.9547-4-53-0007.7 \quad 6804.40$ MMCA50R $04-33.4450-111.7616-4-52-000 \quad 7.7 \quad 13003.00$ MMCA5O2R $04-33.4772-11.7681-4-52-0007.87004 .40$ IMCA503R 04-33.3784-111.8020-4-52-000 8.0 12001.90 MMCA504R 04-33.3638-111.7613-4-52-000 7.2 18003.70 MMCA505R 04-33.2914-1111.8629-4-52-000 7.5 23002.20 YMCA506R 04-33.3064-111.7522-4-52-000 7.1 20001.20 MMCA507R $04-33.2780-1$ i $1.7725-4-52-0007.9 \quad 13001.80$ YMCA50BR $04-33.2663-111.8231-4-52-000 \quad 7.8 \quad 14001.70$ YMCA509R 04-33.2620-111 8535-4-52-000 7.531001 .40 YMCA5IOR 04-33.3234-111. (1) YMCA5I3R $04-33.3113-111.9793-4-52-0007.623001 .90$ YMCA5I YMCASI4R 04-33.3448-111.9624-4-52-000 7.2 30003.60 MMCASISR $04-33.3619-111.9458-4-52-0007.2531002 .60$ MMCASIGR 04-33.3055-111.8270-4-52-000 7.5 28001.70 MMCA5ITR 04-33.3409-111.7835-4-52-000 7.6 18001.60 YMCA5IBR 04-33.3341-111.8239-4-52-000 7.4 22002.90 MMCA5I9R 04-33.3489-111.8934-4-52-000 7.3 23006.80 MMCA5EOR 04-33.3502-111.8677-4-52-000 7.614002 .20 MMCA52IR 04-33.3603-111.8358-4-52-000 $7.7 \quad 17502.90$ YMCASZ2R 04-33.4948-111.8918-4-52-000 8.09002 .70 YMCA523R 04-33.4732-111.7871-4-52-000 7.7. 7503.20 MMCA524R $04-33.4272-111.8258-4-52-000 \quad 7.4 \quad 13003.70$ YMCA525R $04-33.4081-111.8234-4-52-000 \quad 7.6 \quad 11002.10$ YMCA526R $04-33.4075-111.8472-4-52-0008.31100110$ YMCA527R $04-33.4333-111.8781-4-52-000 \quad 6.9 \quad 13004.50$ YMCA528R 04-33.4501-111.8551-4-52-000 7.9 13004.50 YMCA529R 04-33.4108-111.8802-4-52-000 7.9 MMCASTR $04-33.4112-111.9337-4-52-0007.6212002 .50$ MMCA53IR 04-33.4584-111.9157-4-52-000 7.514004 .70 MMCASBTR O4-33.4584-111.9157-4-52-000 7.5 1400 4.70 MCASER O4 MCBSOIR 04 $33.4374-111.5185-4-52-0007.75030$ MMCBSOER $04-33.4636-111.5350-4-52-0007.45303 .60$ MMCBS03R $04-33.4530-111.57 .55-4-7-0007.43743 .10$ MMCB504R 04-33.4326-111.6149-4-52-000 7.6 7202.40 MMCB505R 04-33.3728-111.5766-4-52-000 8.4 2700.90 MMCBEO6R 04-33.2637-111.5632-4-52-000 $7.6 \quad 9001.30$ MMCB5OTR 04-33.2637-111.5367-4-52-000 8.4 MMCB508R 04-33.2632-101.6086-4-52-000 7.6 YMCB509R $04-33.3072-111.5833-4-52-0007.8 \quad 3501.60$ YMCB5IOR 04-33.2919-111.6174-4-52-000 $7.9 \quad 5701.60$ YMCB5IIR $04-33.3855-111.6329-4-52-000 \quad 8.0 \quad 9002.40$ YMCB512R $04-33.3858-111.5272-4-52-000 \quad 7.5 \quad 3720 \quad 1.90$ MMCB513R 04-33.3361-111.6447-4-52-000 9.0 396 0.80 YMCB514R $04-33.4004-111.6231-4-52-000 \quad 7.7 \quad 8402.40$ YMCE515R $04-33.3785-111.6186-4-5^{\circ}-000 \quad 8.2 \quad 4601.70$ $4 M C B 516 R$ 04-33.3194-111.6068-4-52-000 9.8 -1MCB517R $04-33.2712-111.6341-4-52-000 \quad 7.5 \quad 9901.10$

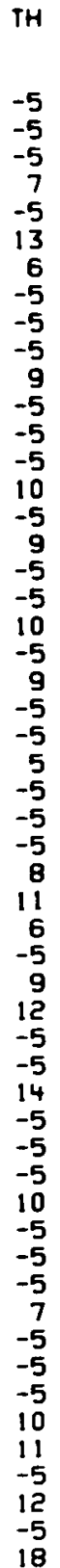

CA CE CO CA

$\mathrm{CU}$ 
APPENDIX 11 TABULATION OF KEY FIELD MEASUREMENTS AND ANALYTICAL DATA--- GROUND WATER---

FE LI MN MO NB NI P SC SE SR TI $V Y$ ZN AND ZR CONCENTRATIONS IN PPB. CA K MG NA AND SI CONCENTRATIONS IN PPM.

SRL 1.D.

YMBE514R

MMBE $16 R$

MMBE517R

MMBE $518 R$

MMCA5OIR

MMCA50วR

YMCA503R

MMCA504R

MMCA505R

MMCA506R

YMCA507R

MMCA508R

YMCASIOR

MMCASIIR

MMCASILR

MMCASI 3R

MMCA5 I4R

MMCA5 I5R

MMCA5ITR

MMCA5IBR

MMCA5ISR

MMCA5PIR

MMCA522R

MMCA523R

MMCA524R

MMCA525R

MMCA526R

MMCA52BR

MMCA529R

YMCA530R

MMCA53IR

YMCA532R

MMCB5OIR

MMCB502R

$M M C B 504 R$

MMCB505R

MMCB506R

MMCB507R

MMCB508R

MMCB509R

MMCBSIOR

MMCB5I IR

MMCB5 $13 R$

MMCB514R

MMCB515R

MMCB516R
MMCB517R

$\begin{array}{rrrr}\text { FE } & K & L 1 & M G \\ 82 & 1.4 & 100 & 1.8 \\ 16 & 15.0 & 15 & 27.9 \\ -10 & 9.7 & 274 & 27.2 \\ -10 & 2.9 & 13 & 28.8 \\ 796 & 7.2 & 6 & 40.9 \\ -10 & 5.5 & 134 & 23.7 \\ 37 & 2.7 & 92 & 11.3 \\ -10 & 5.5 & 145 & 15.5 \\ -10 & 8.5 & 282 & 29.0 \\ -10 & 7.8 & 225 & 51.2 \\ -10 & 8.0 & 196 & 38.9 \\ -10 & 7.5 & 108 & 33.5 \\ -10 & 7.6 & 133 & 37.5 \\ -10 & 9.0 & 246 & 71.3 \\ -10 & 6.8 & 251 & 33.1 \\ -10 & 5.9 & 165 & 38.0 \\ -10 & 5.1 & 207 & 40.3 \\ -10 & 6.2 & 117 & 35.3 \\ -10 & 6.5 & 185 & 52.9 \\ -10 & 8.3 & 186 & 77.3 \\ 14 & 7.2 & 257 & 49.4 \\ -10 & 4.9 & 163 & 17.6 \\ -10 & 7.9 & 235 & 54.6 \\ -10 & 5.8 & 209 & 40.5 \\ -10 & 5.7 & 135 & 39.8 \\ -10 & 6.7 & 179 & 45.3 \\ -10 & 3.8 & 118 & 16.2 \\ -10 & 3.2 & 107 & 11.9 \\ -10 & 4.8 & 103 & 24.7 \\ -10 & 4.7 & 82 & 26.7 \\ -10 & 2.9 & 121 & 4.3 \\ -10 & 7.3 & 129 & 23.6 \\ 24 & 4.7 & 116 & 15.6 \\ -10 & 4.8 & 115 & 22.8 \\ -10 & 6.1 & 207 & 32.8 \\ -10 & 5.6 & 177 & 29.6 \\ -10 & 6.2 & 181 & 50.4 \\ 65 & 2.4 & 30 & 29.4 \\ -10 & 2.1 & 74 & 16.0 \\ -10 & 3.1 & 43 & 12.3 \\ 108 & 4.4 & .52 & 15.6 \\ 118 & 3.2 & 41 & 0.9 \\ 37 & 4.4 & 29 & 15.7 \\ -10 & 4.3 & 29 & 12.6 \\ 13 & 5.5 & 54 & 13.6 \\ 25 & 4.0 & 27 & 9.2 \\ 324 & 4.7 & 41 & 9.8 \\ -10 & 3.8 & 134 & 1.5 \\ 14 & 25.0 & 1550 & 19.7 \\ 224 & 9.5 & 406 & 6.8 \\ -10 & 9.5 & 269 & 8.6 \\ 103 & 3.5 & 42 & 1.1 \\ -10 & 2.7 & 65 & 4.5 \\ -10 & 5.2 & 52 & 18.5 \\ & & & \end{array}$

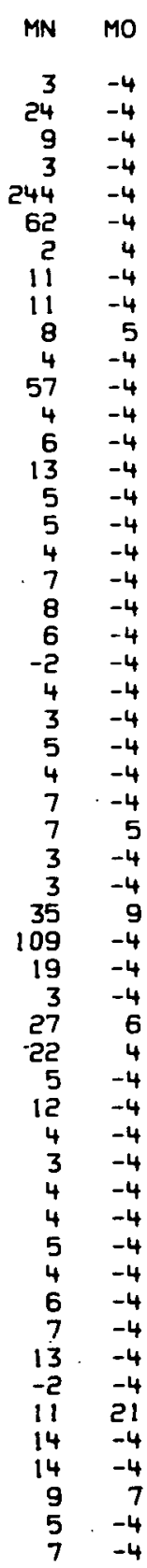

NA NB NI

$\begin{array}{rr}115.7 & -4 \\ 26.5 & -4 \\ 366.2 & -4 \\ 18.7 & -4 \\ 18.4 & -4 \\ 190.9 & \\ 129.3 & -4 \\ 155.2 & 1 \\ 229.2 & -4 \\ 252.9 & -4 \\ 179.5 & \\ 94.2 & -4 \\ 97.1 & -4 \\ 244.5 & -4 \\ 351.4 & -4 \\ 191.8 & -4 \\ 313.1 & -4 \\ 173.6 & -4 \\ 303.8 & \\ 277.2 & -4 \\ 252.0 & -4 \\ 206.5 & \\ 238.8 & -4 \\ 309.3 & -4 \\ 154.5 & -4 \\ 193.1 & -4 \\ 128.9 & -4 \\ 137.3 & -4 \\ 162.4 & -4 \\ 106.5 & -4 \\ 173.8 & \\ 181.8 & -4 \\ 163.2 & 1 \\ 123.9 & 11 \\ 267.4 & 4 \\ 171.4 & \\ 222.9 & \\ 66.5 & -4 \\ 36.6 & \\ 28.6 & -4 \\ 56.6 & -4 \\ 66.2 & \\ 61.1 & -4 \\ 57.6 & -4 \\ 69.4 & 1 \\ 35.1 & -4 \\ 52.2 & -4 \\ 120.7 & -4 \\ 392.9 & 1 \\ 152.7 & -4 \\ 128.1 & -4 \\ 72.5 & -4 \\ 49.8 & \\ 58.2 & -4 \\ \end{array}$

P SC

SE

-4
-4
-4
-4
10
-4
7
8
4
-4
-4
-4
-4
-4
9
-4
-4
-4
-4
-4
4
7
-4
-4
11
-4
-4
-4
-4
-4
-4
-4
4
-4
-4
5
4
4
-4
-4
-4
-4
7
7
-4
-4
-4
-4
-4
-4
4
5
6
6

$\begin{array}{rrrrr}-40 & -1.0 & 0.8 & 6.3 & 80\end{array}$ $\begin{array}{lllll}276 & -1.0 & -0.2 & 10.3 & 391\end{array}$ $\begin{array}{lllll}-40 & 1.0 & -0.2 & 10.6 & 965\end{array}$ $\begin{array}{lllll}-40 & 1.0 & -0.2 & 22.1 & 158\end{array}$ $\begin{array}{rrrrr}59 & 1.0 & -0.2 & 12.4 & 792\end{array}$ $\begin{array}{lllll}-40 & -1.0 & -0.2 & 10.3 & 298\end{array}$ $\begin{array}{rrrrr}-40 & -1.0 & -0.2 & 14.7 & 584 \\ -40 & -1.0 & 0.2 & 17.3 & 2350\end{array}$ $\begin{array}{rrrrr}-40 & -1.0 & 0.2 & 17.3 & 2350 \\ -40 & -1.0 & -0.2 & 13.4 & 2092\end{array}$ $\begin{array}{lllll}-40 & -1.0 & -0.2 & 13.4 & 2092\end{array}$ $\begin{array}{lllll}-40 & 1.0 & -0.2 & 10.4 & 2042\end{array}$ $\begin{array}{lllll}-40 & -1.0 & -0.2 & 13.4 & 1737\end{array}$ $\begin{array}{lllll}-40 & -1.0 & -0.2 & 14.4 & 1842\end{array}$ $\begin{array}{lllll}-40 & 1.0 & -0.2 & 10.4 & 3666\end{array}$ $\begin{array}{lllll}-40 & 1.0 & -0.2 & 12.7 & 1539\end{array}$ $\begin{array}{lllll}-40 & -1.0 & -0.2 & 13.0 & 1325\end{array}$ $-40-1.0-0.5 \quad 9.01569$ $\begin{array}{lllll}-40 & 1.0 & -0.2 & 10.9 & 1868\end{array}$ $\begin{array}{lllll}-40 & 1.0 & -0.2 & 11.3 & 2758\end{array}$ $\begin{array}{lllll}-40 & -1.0 & -0.2 & 14.6 & 2943\end{array}$

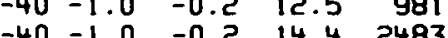
$\begin{array}{rrrrr}-40 & -1.0 & -0.2 & 14.4 & 2483\end{array}$ $\begin{array}{lllll}-40 & 1.0 & 0.4 & 10.7 & 1268\end{array}$ $\begin{array}{lllll}-40 & 1.0 & 0.3 & 11.8 & 1260\end{array}$ $\begin{array}{rrrr}-40 & -1.0 & 0.3 & 12.3 \\ -40 & -1.0 & 0.2 & 11.3\end{array}$ $\begin{array}{lllll}-40 & -1.0 & 0.2 & 9.0 & 279\end{array}$ $\begin{array}{lllll}-40 & -1.0 & 0.4 & 11.2 & 753\end{array}$ $\begin{array}{lllll}-40 & -1.0 & 0.2 & 13.1 & 743\end{array}$ $\begin{array}{lllll}-40 & -1.0 & 0.2 & 7.7 & 211\end{array}$ $\begin{array}{lllll}745 & -1.0 & 0.3 & 13.7 & 675\end{array}$

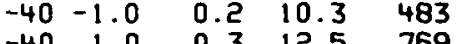
$\begin{array}{rrrrr}-40 & 1.0 & 0.3 & 12.5 & 769 \\ -40 & -1.0 & 0.3 & 12.4 & 1012\end{array}$

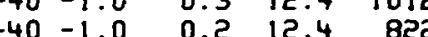
$\begin{array}{lllll}-40 & -1.0 & 0.2 & 11.9 & 2243\end{array}$

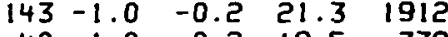
$\begin{array}{lllll}-40 & -1.0 & -0.2 & 18.5 & 338\end{array}$ $\begin{array}{lllll}-40 & -1.0 & -0.2 & 29.8 & 353\end{array}$ $\begin{array}{lllll}-40 & -1.0 & -0.2 & 22.4 & 743\end{array}$ $\begin{array}{lllll}-40 & -1.0 & -0.2 & 17.1 & 129\end{array}$ $\begin{array}{lllll}-40 & -1.0 & -0.2 & 15.1 & 565\end{array}$ $\begin{array}{lllll}-40 & -1.0 & -0.2 & 17.7 & 446 \\ -40 & -1.0 & -0.2 & 15.9 & 723\end{array}$ $\begin{array}{lllll}-40 & -1.0 & -0.2 & 15.9 & 723 \\ -40 & -1.0 & -0.2 & 17.7 & 402\end{array}$

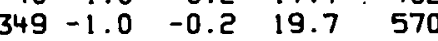
$\begin{array}{lllll}-40 & -1.0 & -0.2 & 16.7 & 210\end{array}$ $\begin{array}{lllll}-40 & 1.0 & -0.2 & 21.3 & 2548\end{array}$ $\begin{array}{lllll}-40 & -1.0 & -0.2 & 10.4 & 609\end{array}$ $\begin{array}{lllll}-40 & -1.0 & -0.2 & 20.7 & 819 \\ -40 & -1.0 & -0.2 & 19.3 & 155\end{array}$ $-40-1.0 \quad-0.2 \quad 18.5 \quad 266$ $\begin{array}{rrrr}-40-1.0 & -0.2 \cdot 18.5 & 266 \\ -40-1.0 & -0.2 \cdot 15.0 \quad 1138\end{array}$

$\begin{array}{rrrrr}1 & V & Y & 2 N & 2 R \\ -2 & 55 & -1 & -4 & -2 \\ 3 & -4 & -1 & -4 & -2 \\ 5 & 5 & -1 & 1158 & -2 \\ 3 & 13 & 1 & 44 & -2 \\ 3 & -4 & -1 & 86 & -2 \\ 3 & 7 & 1 & -4 & -2 \\ 2 & 8 & 1 & 64 & -2 \\ 2 & 10 & -1 & -4 & -2 \\ 3 & 7 & -1 & 920 & -2 \\ 3 & 5 & -1 & 85 & -2 \\ 4 & 6 & -1 & -4 & -2 \\ 3 & -4 & -1 & 511 & -2 \\ 3 & -4 & -1 & 17 & -2 \\ 7 & -4 & -1 & 81 & -2 \\ 4 & -4 & 1 & 1736 & 2 \\ 3 & -4 & -1 & 123 & -2 \\ 4 & 4 & -1 & 304 & -2 \\ 3 & -4 & -1 & 160 & -2 \\ 5 & -4 & -1 & 35 & -2 \\ 6 & 8 & -1 & 44 & -2 \\ 4 & -4 & -1 & -4 & -2 \\ -2 & 6 & 1 & 6 & -2 \\ 4 & -4 & -1 & 552 & -2 \\ 5 & -4 & -1 & 183 & -2 \\ 4 & -4 & -1 & 40 & -2 \\ 3 & -4 & -1 & 18 & -2 \\ -2 & 21 & -1 & -4 & -2 \\ -2 & 4 & -1 & -4 & -2 \\ 2 & 8 & 1 & -4 & -2 \\ 2 & 12 & 1 & -4 & -2 \\ 3 & 46 & -1 & -4 & -2 \\ 2 & 9 & -1 & -4 & -2 \\ 3 & 7 & -1 & -4 & -2 \\ 3 & 13 & 1 & -4 & 2 \\ 3 & 8 & -1 & -4 & -2 \\ 2 & 7 & -1 & -4 & -2 \\ 4 & 14 & 1 & 129 & 3 \\ 2 & 12 & -1 & 211 & -2 \\ 2 & 5 & -1 & 1867 & -2 \\ 3 & 10 & -1 & 848 & -2 \\ 4 & 7 & -1 & 378 & -2 \\ -2 & 21 & -1 & 265 & -2 \\ 4 & 8 & -1 & 159 & -2 \\ 3 & 12 & -1 & 75 & -2 \\ 3 & 13 & -1 & 77 & -2 \\ 2 & 11 & -1 & 158 & -2 \\ 15 & 22 & -1 & 41 & -2 \\ 2 & 13 & -1 & 19 & -2 \\ 8 & 8 & -1 & 377 & -2 \\ 6 & 5 & 1 & 44 & 2 \\ 5 & 9 & -1 & 209 & -2 \\ 3 & 22 & 1 & 59 & 3 \\ 2 & 28 & -1 & 79 & -2 \\ 4 & 10 & 1 & 34 & 3 \\ & & & & \end{array}$



ELEMENTAL CONCENTRATIIJNS IN PPB. ALK IN MEQIL. COND IN UM/CM.

SRL I.D.

DOE 1.D.

PH COND ALK

TH AG

YMCB5' $3 R \quad 04-33.2660-: 11.6853-4-52-000 \quad 7.5 \quad 8700.50$ YMCBSISR $04-33.2598-111 . " 1374_{i}-+-52-000 \quad 7.3 \quad 2300 \quad 1.50$ YMC B520R $04-33.2919-111.7440-+-52-000 \quad 7.7 \quad 8501.30$ MMCB52IR $04-33.3078-111.6881-4-52-000 \quad 7.0 \quad 3210 \quad 1.30$ YMCB522R $04-33.3327-111.6875-4-52-0009.2$ 282 1.50 YMCB523R $04-33.3494-111.7172-4-52-000 \quad 7.3 \quad 24703.20$ YMCB524R 04-33.4219-111.5290-4-52-000 6.7 820 1.30 YMCB525R 04-33.3933-111.5697-4-52-000 7.6 9202.60 YMCB526R $04-33.3936-111.6843-4-52-000$ 8.1 6201.30 YMCB527R $04-33.3815-111.6685-4-52-0007.92511 .30$ MMCB528R $04-33.3090-111.6411-4-52-0008.1 \quad 420.1 .60$ YMCB529R $04-33.3650-111.7338-4-52-0008.4 \quad 52011.90$ MMCBSERR $04-33.3650-111.7338-4-52-0008.452011 .90$ MMCBS3OR 04-33.4537-111.7183-4-52-000 7.7 1000 1.90 YMCB53IR O4-33.4144-111.7370-4-52-000 $8.0 \quad 13002.00$ MMCBS3ER 04-33.3996-111.6687-4-5e-000 7.8 1000 1.10 YMCB533R 04-33.4436-.11.6561-4-52-000 7.9 600 1.90 YMCB534R 04-33.4588-.11.6857-4-52-000 7.7 560 1.60 YMCB535R 04-33.4659-?11.7356-4-52-000 7.4 13102.90 YMCC50IR 04-33.3603-111.4714-4-52-000 9.5 3611.70 YMCC502R: $04-33.3810-111.4333-4-52-6007.95102 .60$ YMCC503R 04-33.2927-111.3810-4-52-000 7.6 281 2.40 YMCC504R 04-33.2594-111.3359-4-52-000 7.2 5703.20 YMCC505R $04-33.3006-111.2933-4-52-0007.3 \quad 10724.00$ YMCC506R 04-33.2954-111.3205-4-52-000 7.3 5603.40 $4 M C C 507804-33.3522-111.3412-4-52-000 \quad 6.8 \quad 4471.50$ YMCC508R 04-33.4577-111.4872-4-52-000 7.1 10003.00 YMCC5IO $04-33.4136-111.4679-4-52-0006.8 \quad 5604.00$ पMCC5IOA $04-33.4136-111.4819-4-52-000$ 8.1 2571.40 YMCC

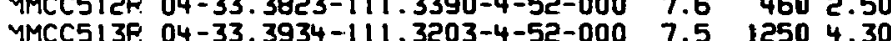
MMCC S1 उP. $04-33.3934-111.3203-4-52-000$ 7.5 12504.30 MMCCSI4R O4-33.4993-111.2991-4-52-000 7.11301 .00 YMCDSOIR O4-33.2805-111.1497-4-52-000 6.8 1210 5.50 MMCDSO2R $04-33.2710-111.1833-4-52-0007.36904 .80$ MMCD503R 04-33.2987-111.2483-4-52-000 8.5 980 2.30 YMCD504R 04-33.2994-111.1971-4-53-000 7.2 8803.90 YMCO505R 04-33.3213-111. 1654-4-53-000 8.4 1110.30 YMCO506R 04-33.3390-111 .1353-4-52-000 7.0 7902.40 YMCD507R 04-33.3271-111.2309-4-52-000 7.3 7093.20 YMCD508R 04-33.3586-111.2082-4-52-000 7.4 810 5.50 YMCD509R 04-33.3782-111.1922-4-52-000 7.8 11705.70 YMCD5 IOR $04-33.4194-111.0330-4-53-000$ 8.0 2951.40 YMCDEIIR 04-33.4371-111. $0711-4-53-0007.3 \quad 6003.30$ YMCDSI $2 R \quad 04-33.4281-111.0865-4-53-000 \quad 8.3 \quad 7004.10$ MMCD513R 04-33.4275-111.0176-4-53-000 8.1 2250.80 YMCDSI4R 04-33.4618-11:0040-4-52-0007.9 6003.30 YMCD5I5P $04-33.3403-11.0080-4-52-0006.1$ पMCO5 $04-33.3397-111.0527-4-53-0003.4 \quad 3400.60$ पMCD5172 $04-33.3403-111.0951-4-53-000$ 7. 600 2.90 4MCD5IOR 04-33.3580-11.0953-4-53-000 7.9 71090 YMCDSISR 04-33.4761-111.0465-4-53-000 8.5 525 4.30 MMCOSISR $04-33.4761-111.0465-4-53-000$ 8.5 5254.30 MMCESOIR $04-33.3070-110.7781-4-53-0007.62401 .15$ YMCE503R $04-33.4160-113.8331-4-52-0006.6 \quad 7001.10$

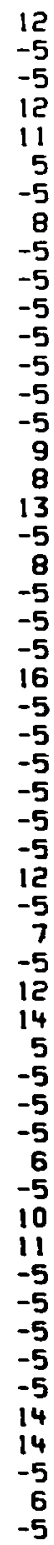

12
-5
-5
12
11
5
-5
8
-5
-5
-5
-5
-5
-5
-5
9
8
13
-5
8
-5
-5
-5
16
-5
-5
-5
-5
-5
-5
12
-5
7
-5
12
14
5
-5
-5
-5
6
-5
10
11
-5
-5
-5
-5
-5
14
14
-5
6
-5
AL AS B

CA CE

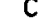

$\begin{array}{rr}95 & 59 \\ 151 & 156 \\ 91 & 20 \\ 120 & 361 \\ 154 & 54 \\ 1396 & 70 \\ 317 & 80 \\ 341 & 60 \\ 171 & 23 \\ 108 & 7 \\ 73 & 26 \\ 63 & 12 \\ 138 & 41 \\ 191 & 77 \\ 431 & 32 \\ 171 & 27 \\ 87 & 19 \\ 171 & 64 \\ 73 & 5 \\ 138 & 137 \\ 135 & 8 \\ 53 & 45 \\ 64 & 30 \\ 49 & 24 \\ 75 & 10 \\ 131 & 31 \\ 67 & 33 \\ 121 & 7 \\ 52 & 133 \\ 49 & 33 \\ 66 & 13 \\ 22 & 11 \\ 76 & 7 \\ 67 & 18 \\ 64 & 31 \\ 94 & 6 \\ 12 & 22 \\ 36 & 21 \\ 47 & 17 \\ 77 & 30 \\ 72 & 24 \\ 28 & 45 \\ 39 & 61 \\ 32 & 51 \\ 51 & 28 \\ 125 & 121 \\ 74 & 63 \\ 28 & 35 \\ 42 & 23 \\ 161 & 25 \\ 51 & 517 \\ 21 & 41 \\ 35 & 67 \\ 53 & 28 \\ & \end{array}$

$\begin{array}{rrr}-1 & 73 & -30 \\ -1 & 272 & -30 \\ -1 & 51 & -30 \\ -1 & 427 & -30 \\ -1 & 61 & -30 \\ -1 & 306 & -30 \\ -1 & 144 & -30 \\ -1 & 71 & -30 \\ -1 & 33 & -30 \\ -1 & 18 & -30 \\ -1 & 45 & -30 \\ -1 & 23 & -30 \\ -1 & 51 & -30 \\ -1 & 34 & -30 \\ -1 & 45 & -30 \\ -1 & 43 & -30 \\ -1 & 42 & -30 \\ -1 & 70 & -30 \\ -1 & 7 & -30 \\ -1 & 47 & -30 \\ -1 & 24 & -30 \\ 2 & 45 & -30 \\ -1 & 151 & -30 \\ -1 & 96 & -30 \\ -1 & 43 & -30 \\ -1 & 68 & -30 \\ -1 & 84 & -30 \\ -1 & 20 & -30 \\ -1 & 53 & -30 \\ -1 & 57 & -30 \\ -1 & 73 & -30 \\ -1 & 16 & -30 \\ -1 & 162 & -30 \\ -1 & 97 & -30 \\ -1 & 132 & -30 \\ -1 & 98 & -30 \\ 1 & 7 & -30 \\ -1 & 109 & -30 \\ -1 & 81 & -30 \\ -1 & 114 & -30 \\ -1 & 153 & -30 \\ 1 & 33 & -30 \\ 2 & 86 & -30 \\ -1 & 107 & -30 \\ -1 & 33 & -30 \\ -1 & 65 & -30 \\ -1 & 35 & -30 \\ -1 & 95 & -30 \\ -1 & 100 & -30 \\ 1 & 73 & -30 \\ -1 & 75 & -30 \\ -1 & 38 & -30 \\ -1 & 75 & -30 \\ 1 & 94 & -30\end{array}$

$\begin{array}{rrr}-2 & 5 & 5 \\ -2 & -4 & 21 \\ -2 & 11 & 6 \\ 3 & 4 & 19 \\ 4 & 33 & 8 \\ -2 & 12 & 21 \\ 68 & -4 & 21 \\ 28 & 8 & 22 \\ 4 & 15 & 9 \\ -2 & 14 & 5 \\ -2 & 6 & 9 \\ -2 & 14 & 3 \\ -2 & -4 & 14 \\ -2 & -4 & 5 \\ -2 & 7 & 7 \\ -2 & 7 & 5 \\ -2 & 4 & 6 \\ -2 & 5 & 4 \\ -2 & 6 & 2 \\ -2 & 9 & 10 \\ -2 & -4 & 5 \\ -2 & -4 & 15 \\ -2 & -4 & 28 \\ -2 & 6 & 29 \\ -2 & -4 & 10 \\ -2 & -4 & 32 \\ -2 & -4 & 20 \\ -2 & 13 & 7 \\ -2 & -4 & 18 \\ -2 & -4 & 13 \\ -2 & 4 & 11 \\ -2 & -4 & 10 \\ -2 & -4 & 12 \\ -2 & -4 & 4 \\ -2 & 4 & 6 \\ -2 & 5 & 4 \\ -2 & 4 & 17 \\ -2 & -4 & 3 \\ -2 & -4 & 57 \\ -2 & -4 & 9 \\ -2 & -4 & 10 \\ -2 & -4 & 8 \\ 5 & 6 & 7 \\ -2 & 5 & 5 \\ -2 & -4 & 7 \\ -2 & -4 & 2 \\ -2 & -4 & 39 \\ -2 & -4 & 14 \\ -2 & -4 & 14 \\ 6 & 4 & 25 \\ 4 & 6 & 5 \\ -2 & -4 & 7 \\ -2 & -4 & 21 \\ 5 & -4 & 75 \\ & & \end{array}$


APPENDIX II TABULATION OF KEY FIELD MEASUREMENTS AND ANALYTICAL DATA--- GROUND WATER---

FE LI MN MO NB NI P SC SE SR TI $\checkmark Y$ ZN AND ZR CONCENTRATIONS IN PPB. CA K MG NA AND SI CONCENTRATIONS IN PPM.

SRL 1.0

MMCB5 I8R

MMCBS I IR

MMCB52OR

MMCBSE2R

MMCB523R

YMCB524R

YMCB525R

MMCB527R

MMCB52BR

MMCB529R

MMCBS3OR

MMCB532R

MMCB533R

MMCB534R

MMCBS35R

YMCC5OZR

MMCC503R

$M M C C 504 R$

MMCC505R

MMCC506R

MMCC50BR

MMCC509R

MMCC5I OR

MMCCSIIR

MMCC51 3R

MMCC514R

MMCDSO $1 R$

MMCDSO2R

MMCDSO4R

MMCD505R

MMCD506R

MMCD507R

MMCD508R

MMCO509R

MMCDSIIR

MMCD512R

MMCD513R

MMCD514R

MMCD515R

MMCDSI6R

MMCD517R

MMCD5 I8R

MMCDSISR

MMCESOIR

MMCE502R
FE $K$ LI MG

$\begin{array}{rr}-10 & 5.3 \\ -10 & 7 \\ -10 & 5.8 \\ 142 & 9.4 \\ -10 & 2.3 \\ -10 & 10 \\ 76 & 3 \\ -10 & 11 \\ 117 & 6 \\ 13 & 3 \\ 68 & 3 \\ 13 & 4 \\ 135 & 4\end{array}$

135
-10

$-10$

-10
18

18
-10
-10

-10
-10
23

255

113.

$-10 \quad 3.6$

$-10-10.7$

$\begin{array}{ll}-10 & 5.5\end{array}$

$-10 \quad 2.4$

$\begin{array}{ll}-10 & 1.9 \\ -10 & 2.4\end{array}$

$-10 \quad 4.4$

$\begin{array}{rr}-10 & 2.9 \\ 56 & 3.7\end{array}$

$\begin{array}{rl}56 & 2.7 \\ -10 & 2.7\end{array}$

2.7

$17 \quad 2.9$

$\begin{array}{ll}-10 & 1.4\end{array}$

$\begin{array}{lll}-181 & 2.5\end{array}$

$263 \quad 2.0$

$885 \quad 1.0$

$44 \quad 1.2$

$202 \quad 3.4$

$-10 \quad 1.2$

$\begin{array}{rr}41 & 3.9\end{array}$

$-10 \quad 25.3$

$63 \quad 3.9$

$19 \quad 6.3$

$207 \quad 5.0$

118

1529
489

$\begin{array}{rr}75 & 15.6 \\ 101 & 51.0 \\ 147 & 18.0 \\ 91 & 93.4 \\ 48 & 12.1 \\ 235 & 76.1 \\ 53 & 28.8 \\ 212 & 12.1 \\ 129 & 5.1 \\ 82 & 1.7 \\ 40 & 8.0 \\ 101 & 4.9 \\ 104 & 16.4 \\ 246 & 6.5 \\ 395 & 3.9 \\ 151 & 9.4 \\ 69 & 9.5 \\ 126 & 19.9 \\ 74 & 1.7 \\ 24 & 19.1 \\ 29 & 4.7 \\ 24 & 9.4 \\ 19 & 30.9 \\ 12 & 21.9 \\ 26 & 5.3 \\ 51 & 27.5 \\ 50 & 21.1 \\ 16 & 4.5 \\ 22 & 19.7 \\ 25 & 15.8 \\ 91 & 33.8 \\ 2 & 3.2 \\ 11 & 67.1 \\ 33 & 20.1 \\ 13 & 33.4 \\ 41 & 29.5 \\ 8 & 2.4 \\ 28 & 38.9 \\ 10 & 25.7 \\ 15 & 48.4 \\ 59 & 44.8 \\ 10 & 11.7 \\ 13 & 33.0 \\ 39 & 32.1 \\ 9 & 8.5 \\ 58 & 9.8 \\ 10 & 10.8 \\ 7 & 34.6 \\ 10 & 23.1 \\ 5 & 27.6 \\ 23 & 20.2 \\ 9 & 11.9 \\ 4 & 21.6 \\ 17 & 19.0 \\ & \\ & \end{array}$

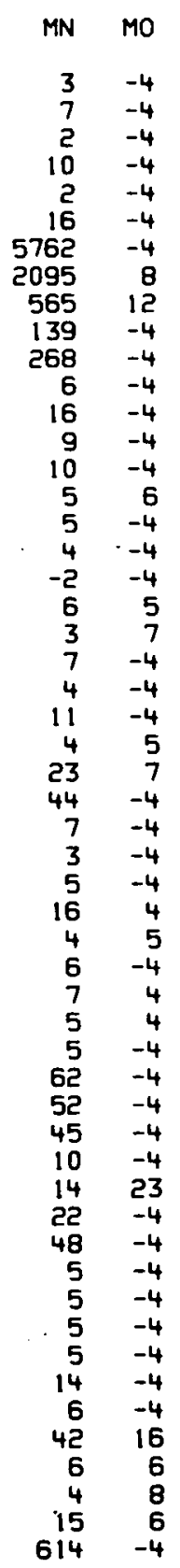

NA

78.2

111.5

90.1

74.4

198.1

86.3
96.4

96.4

102.7

50.3

95.1

157.4

149.8

107.6

76.7

174.9

111.2

90.5
49.3

50.

42.5

50.4

42.6

47.4

38.8

32.4
128.9

128.9

46.3
27.8

35.1

57.6

2.8

25.6

34.0

86.4

9.1
12.7

(1)

11.9

19.8

12.3

28.6

74.9

22.5
19.6

19.6
25.9

59.4

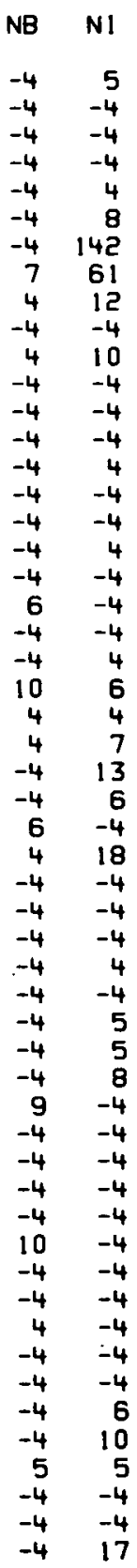

$\begin{array}{lllll}-40 & -1.0 & -0.2 & 15.1 & 839\end{array}$ $\begin{array}{lllll}-40 & 1.0 & 0.2 & 14.3 & 3360\end{array}$

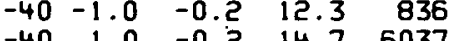
$\begin{array}{lllll}-40 & -1.0 & -0.2 & 11.0 & 746\end{array}$ $\begin{array}{lllll}-40 & 1.0 & -0.2 & 17.3 & 3955\end{array}$ $\begin{array}{lllll}-40 & 1.0 & -0.2 & 12.9 & 1908\end{array}$ $\begin{array}{lllll}-40 & -1.0 & -0.2 & 19.5 & 1060\end{array}$ $\begin{array}{lllll}-40 & -1.0 & -0.2 & 16.2 & 418\end{array}$ $\begin{array}{lllll}-40 & -1.0 & -0.2 & 14.8 & 148\end{array}$ $\begin{array}{lllll}-40 & -1.0 & -0.2 & 14.5 & 448\end{array}$ $\begin{array}{lllll}-40 & -1.0 & -0.2 & 16.1 & 283\end{array}$ $\begin{array}{lllll}-40 & -1.0 & -0.2 & 11.7 & 555\end{array}$ $\begin{array}{lllll}-40 & -1.0 & -0.2 & 16.3 & 419\end{array}$ $\begin{array}{lllll}-40 & -1.0 & -0.2 & 18.1 & 530\end{array}$ $\begin{array}{lllll}-40 & -1.0 & -0.2 & 12.0 & 401\end{array}$ $\begin{array}{lllll}-40 & -1.0 & -0.2 & 8.9 & 335\end{array}$ $\begin{array}{lllll}-40 & 1.0 & -0.2 & 10.9 & 632\end{array}$ $\begin{array}{lllll}-40 & -1.0 & -0.2 & 14.3 & 58 \\ -40 & -1.0 & -0.2 & 19.3 & 2977\end{array}$ $\begin{array}{lllll}-40 & -1.0 & -0.2 & 19.3 & 2977\end{array}$ $\begin{array}{lllll}-40 & -1.0 & -0.2 & 19.7 & 257\end{array}$ $\begin{array}{llll}-40 & 2.0 & -0.2 & 19.7\end{array}$ $\begin{array}{llll}-40 & 1.0 & -0.2 & 17.7\end{array}$ $\begin{array}{lllll}-40 & 1.0 & -0.2 & 19.2 & 386\end{array}$ $\begin{array}{lllll}66 & -1.0 & -0.2 & 24.8 & 658\end{array}$ $\begin{array}{lllll}-40 & -1.0 & -0.2 & 19.0 & 737\end{array}$ $\begin{array}{lllll}-40 & -1.0 & -0.2 & 12.5 & 144\end{array}$ $\begin{array}{lllll}-40 & -1.0 & -0.2 & 19.8 & 24\end{array}$ $\begin{array}{llll}75 & -1.0 & -0.2 & 23.1\end{array}$

$88-1.0 \quad-0.2515 .4$

$\begin{array}{llll}-40 & -1.0 & 0.4 & 32.5\end{array}$

$\begin{array}{llll}-40 & -1.0 & 0.2 & 17.4\end{array}$

$\begin{array}{llll}-40 & 1.0 & 0.3 & 11.4\end{array}$

$72 \quad 1.0 \quad 0.3 \quad 17.5$

$\begin{array}{llll}-40 & -1.0 & 0.2 & 11.5\end{array}$

$\begin{array}{llll}-40 & -1.0 & 0.4 & 14.6\end{array}$

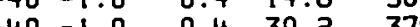

$\begin{array}{lllll}-40 & 1.0 & 0.4 & 18.7 & 1744\end{array}$

$\begin{array}{lllll}-40 & -1.0 & 0.4 & 15.0 & 124\end{array}$

$\begin{array}{lllll}-40 & 2.0 & 0.5 & 11.5 & 179\end{array}$

$\begin{array}{llll}-40 & -1.0 & 0.3 & 16.1\end{array}$

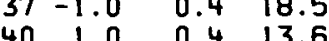

$\begin{array}{llll}181 & -1.0 & 0.3 & 15.0\end{array}$

$\begin{array}{lllll}-40 & -1.0 & -0.2 & 18.9 & 24\end{array}$

$\begin{array}{llll}52 & -1.0 & -0.2 & 13.9\end{array}$

$47 \quad 1.0 \quad-0.2 \quad 21.4$

$57-1.0 \quad-0.2 \quad 17.1$

$-40-1.0 \quad-0.2 \quad 12.8$

$\begin{array}{llll}-40 & -1.0 & 0.3 & 13.6 \\ -40 & -1.0 & 0.2 & 12.1\end{array}$

$\begin{array}{rrrrr}\mathrm{T} I & V & Y & Z N & Z R \\ 3 & 12 & 1 & 25 & -2 \\ 6 & -4 & -1 & 53 & -2 \\ 3 & 10 & -1 & 40 & -2 \\ 11 & -4 & 1 & 646 & -2 \\ 2 & 63 & 1 & 75 & -2 \\ 2 & 5 & 1 & 1226 & -2 \\ 8 & -4 & -1 & 25 E 3 & -2 \\ 5 & 12 & 1 & 7969 & 3 \\ 3 & 12 & -2 \\ 3 & 14 & -1 & 691 & -2 \\ 2 & 18 & -1 & 213 & -2 \\ 2 & -4 & -1 & 274 & -2 \\ 2 & 10 & -1 & 40 & -2 \\ 2 & -4 & -1 & 147 & -2 \\ 8 & -2 & -2 & -2 \\ 3 & 14 & -1 & 28 & -2 \\ 3 & 8 & -1 & 60 & -2 \\ 3 & 11 & -1 & 36 & 2 \\ 3 & -4 & -1 & 39 & -2 \\ 3 & 5 & 1 & -4 & -2 \\ -2 & 33 & -1 & 51 & -2 \\ 2 & 24 & -1 & 820 & 3 \\ 2 & 16 & -1 & 178 & -2 \\ 3 & 4 & 2 & 276 & -2 \\ 6 & 4 & -1 & 130 & -2 \\ 3 & 8 & 1 & 543 & 3 \\ 2 & -4 & -1 & 2015 & -2 \\ 3 & 5 & -1 & 1099 & -2 \\ 2 & -4 & -1 & 1619 & -2 \\ 2 & 48 & -1 & 29 & -2 \\ 2 & 11 & -1 & 125 & -2 \\ -2 & 4 & -1 & 450 & -2 \\ 3 & 13 & 1 & 1111 & 2 \\ 4 & -4 & -1 & 186 & -2 \\ 4 & 13 & 1 & 913 & -2 \\ 4 & -4 & -1 & 231 & -2 \\ 6 & -4 & 1 & -4 & -2 \\ 4 & 18 & 1 & -4 & 2 \\ 39 & 12 & 3 & 12 & 2 \\ 10 & -4 & -1 & 23 & -2 \\ 3 & -4 & -1 & 950 & -2 \\ 5 & 16 & -1 & 27 & -2 \\ 5 & -4 & -1 & 72 & -2 \\ 8 & -4 & -1 & 16 & -2 \\ 8 & -4 & 3 & 338 & 4 \\ 5 & 6 & 1 & 57 & 2 \\ 5 & 4 & 1 & -4 & -2 \\ 4 & 4 & 1 & 8 & -2 \\ 2 & 6 & -1 & 261 & -2 \\ 6 & -4 & -1 & 23 & -2 \\ 5 & 7 & 1 & 6 & -2 \\ 8 & 19 & 2 & 20 & 4 \\ 8 & 15 & 1 & 16 & 2 \\ 4 & -4 & -1 & -4 & -2 \\ 4 & 5 & -1 & 2017 & -2 \\ 4 & -4 & -1 & 118 & -2 \\ & & & & \end{array}$


APPENDIX II TABULAT:ON OF KEY FIELD MEASUREMENTS AND ANALYTICAL DATA--- GROUND WATER-FE LI MN MO NB NI P SC SE SR TI $V$ Y ZN AND ZR CONCENTRATIONS IN PPB.

CA K MG NA AND SI CONCENTRATIONS :N PPM.

\begin{tabular}{|c|c|c|c|c|c|c|c|c|c|c|c|c|c|c|c|c|c|c|c|}
\hline SRL I.D. & FE & K & LI & MG & MN & MO & NA & NB & $\mathrm{NI}$ & $P$ & SC & SE & SI & SR & $\mathrm{TI}$ & v & Y & $Z N$ & ZR \\
\hline MMCE504R & 2390 & 2.3 & 11 & 19.4 & 799 & -4 & 36.9 & -4 & 17 & 139 & -1.0 & 0.2 & 10.9 & 415 & 4 & -4 & 1 & 127 & -2 \\
\hline MMCE506R & 1546 & 4.9 & 39 & 24.3 & 112 & -4 & 36.4 & -4 & -4 & 42 & -1.0 & 0.3 & 9.9 & 407 & 5 & -4 & -1 & 170 & -5 \\
\hline MMCE507R & 503 & 5.1 & 28 & 33.7 & 25 & -4 & 31.8 & -4 & 5 & -40 & -1.0 & 0.3 & 10.8 & 289 & 3 & -4 & -1 & 559 & -2 \\
\hline MMCE508R & 1137 & 7.9 & 9 & $88: 6$ & 33 & -4 & 46.7 & -4 & -4 & 71 & -1.0 & 0.2 & 16.6 & 501 & 9 & -4 & $-i$ & -4 & -2 \\
\hline MMCE509R & 7016 & 1.2 & 15 & 21.1 & 79 & 4 & 42.0 & -4 & 5 & 53 & 5.0 & 0.2 & 5.7 & 225 & 14 & -4 & 6 & 678 & 2 \\
\hline MMCE5IOR & 727 & 1.5 & 8 & 19.3 & 14 & 4 & 22.9 & -4 & -4 & 54 & -1.0 & -0.2 & 13.4 & 270 & 10 & 6 & $i$ & 43 & -2 \\
\hline MMCE5IIR & -10 & 2.5 & 10 & 40.8 & 20 & 6 & 21.6 & -4 & 4 & -40 & -1.0 & 0.3 & 13.5 & 546 & 3 & -4 & -1 & 630 & -2 \\
\hline MMCESILR & 155 & 0.9 & 3 & 7.5 & 7 & 13 & 10.5 & -4 & -4 & 43 & -1.0 & -0.2 & 12.6 & 102 & 7 & -4 & $-i$ & 49 & -2 \\
\hline MMCE5 I 3R & 189 & 1.1 & 8 & 4.5 & 8 & 4 & $12: 2$ & 9 & 4 & 107 & -1.0 & -0.2 & 14.3 & 95 & 7 & -4 & 1 & -4 & -2 \\
\hline AMCES I LR & 185 & 0.8 & 5 & 7.9 & 3 & -4 & 17.7 & -4 & 6 & -40 & -1.0 & 0.3 & 11.4 & 189 & 2 & -4 & $i$ & 376 & -2 \\
\hline MMCE5 I5R & 189 & 2.9 & 43 & 64.7 & 10 & -4 & 122.7 & -4 & 6 & -40 & -1.0 & 0.5 & 19.0 & 961 & 9 & 11 & -1 & 2125 & -5 \\
\hline MMCES IGR & 309 & 17.2 & 44 & 42.9 & 4551 & 84 & 91.2 & 8 & 22 & 349 & 1.0 & 0.4 & 15.8 & 1523 & 11 & 4 & $i$ & 129 & 2 \\
\hline MMCE5 I 7R & 64 & 29.8 & 10 & 13.8 & 12 & -4 & 58.4 & 8 & 7 & 593 & -1.0 & 0.5 & 13.5 & 364 & 3 & 9 & -1 & 1816 & 2 \\
\hline MMCE5 I8R & -10 & 1.7 & 29 & 16.4 & 2 & -4 & 33.0 & -4 & -4 & -40 & -1.0 & 0.4 & 17.3 & 991 & 2 & -4 & -1 & 55 & -2 \\
\hline MMCE5। SR & 117 & 1.3 & 8 & 4.6 & 6 & -4 & 10.8 & -4 & -4 & 42 & -1.0 & -0.2 & 11.0 & 117 & 6 & -4 & -1 & 105 & -2 \\
\hline MMCESZOR & -10 & 5.4 & 76 & 81.4 & 5 & -4 & 25.1 & -4 & 7 & -40 & 1.0 & 0.3 & 18.8 & 1205 & 3 & 7 & 1 & 12 & -2 \\
\hline MMCESDIR & -10 & 2.0 & 31 & 34.6 & 4 & 7 & 24.0 & -4 & -4 & -40 & -1.0 & 0.3 & 12.9 & 341 & 2 & -4 & -1 & 209 & -5 \\
\hline MMCE522R & 65 & 1.0 & 75 & 25.4 & 45 & -4 & 7.1 & 4 & 4 & -40 & -1.0 & 0.2 & 20.4 & 320 & 4 & 5 & 6 & 54 & 3 \\
\hline MMCE523R & -10 & 5.8 & 10 & 21.8 & 23 & .7 & 18.6 & -4 & 5 & 214 & -1.0 & 0.4 & 18.9 & 713 & 3 & 5 & 1 & 111 & ? \\
\hline MMCE524R & -10 & 2.1 & 19 & 4.5 & 6 & 5 & 22.5 & 18 & -4 & 545 & 1.0 & 0.6 & 24.4 & 327 & 4 & 5 & $\dot{2}$ & $i i 7$ & 5 \\
\hline MMCE525R & 401 & 1.7 & 6 & 14.0 & 16 & 6 & 56.8 & 6 & 11 & 233 & -1.0 & 0.3 & 18.4 & 235 & 21 & 8 & 1 & -4 & 2 \\
\hline MMCES26R & 88 & 2. 2 & 29 & 38.6 & 12 & 10 & 85.8 & 8 & 6 & 67 & -1.0 & 0.5 & 14.4 & 606 & 3 & 14 & 1 & 58 & 2 \\
\hline MMCE 527R & -10 & 2.4 & 16 & 31.7 & 2 & 9 & 34.3 & -4 & -4 & 64 & -1.0 & 0.4 & 20.9 & 296 & -2 & 16 & 1 & -4 & -2 \\
\hline MMDB501R & -10 & 4.6 & 67 & 17.9 & 7 & -4 & 95.1 & -4 & 4 & -40 & 2.0 & -0.2 & 13.9 & 888 & 6 & 7 & 2 & 8 & -2 \\
\hline YMOB502R & -10 & 5.6 & 96 & 10.7 & 5 & -4 & 86.2 & -4 & 6 & -4.0 & -1.0 & -0.2 & 16.0 & 642 & 3 & 10 & -1 & -4 & -2 \\
\hline MMDB503R & -10 & 5.8 & 112 & 12.6 & 3 & -4 & 96.3 & -4 & -4 & -40 & -1.0 & 0.2 & 16.0 & 737 & 3 & 14 & -1 & -4 & -2 \\
\hline MMOB504R & -10 & 4.5 & 85 & 8.0 & 3 & -4 & 118.9 & 4 & 7 & -40 & 1.0 & -0.2 & 11.1 & 672 & 4 & 28 & -1 & -4 & -2 \\
\hline MMDB505R & 59 & 4.6 & 83 & 9.2 & 5 & -4 & 124.8 & -4 & -4 & -40 & 1.0 & -0.2 & 12.0 & 61 & 4 & 20 & -1 & -4 & -2 \\
\hline MMDB506R & -10 & 5.3 & 89 & 10.3 & 9 & 6 & 93.9 & -4 & -4 & -40 & -1.0 & -0.2 & 15.7 & 603 & 4 & 11 & -1 & -4 & -2 \\
\hline MMDB507R & 94 & 7.3 & 189 & 34.4 & 12 & -4 & 199.0 & -4 & 7 & -40 & 1.0 & -0.2 & 16.0 & 1655 & 9 & -4 & -1 & 6 & -2 \\
\hline YMDB508R & 146 & 10.8 & 229 & 44.1 & 22 & 7 & 213.0 & -4 & 8 & 71 & 1.0 & -0.2 & 15.3 & 2992 & 10 & 6 & 1 & 1557 & -2 \\
\hline MMDB509R & 1081 & 5.0 & 124 & 15.5 & 18 & -4 & 146.2 & -4 & -4 & -40 & -1.0 & $-0 . \overline{2}$ & 11.4 & 1188 & 5 & 9 & -1 & 75 & $-\overline{2}$ \\
\hline MMDB51 OR & -10 & 5.4 & 110 & 20.3 & 5 & -4 & 123.1 & 6 & -4 & -40 & -1.0 & -0.2 & 14.6 & & 4 & 12 & 1 & -4 & -2 \\
\hline MMDB51 IR & -10 & 4.8 & 95 & 14.0 & 2 & -4 & 106.2 & -4 & -4 & -40 & -1.0 & -0.2 & 14.4 & 65 & 3 & 16 & $-i$ & -4 & $-\overline{2}$ \\
\hline MMDB5 I 2R & -10 & 4.3 & 62 & 4.9 & 3 & -4 & 93.2 & -4 & -4 & -40 & -1.0 & -0.2 & 13.4 & 439 & 3 & 10 & $-i$ & -4 & -2 \\
\hline MMOB5I 3R & -10 & 6.2 & 75 & 12.7 & 2 & -4 & 74.6 & -4 & -4 & -40 & -1.0 & -0.2 & & 76 & 3 & 10 & -1 & -4 & -5 \\
\hline MMOB514R & -10 & 5.7 & 55 & 11.3 & 2 & -4 & 75.6 & 4 & 7 & -40 & -1.0 & -0.2 & 15.9 & 61 & 3 & 9 & -1 & -4 & -2 \\
\hline MMDB515R & -10 & 6.0 & 73 & 16.7 & 2 & 5 & 94.4 & -4 & -4 & -40 & -1.0 & 0.5 & 15.0 & & 3 & 7 & -1 & -+ & -2 \\
\hline MMDB516R & -10 & 5.7 & 42 & 11.5 & 2 & -4 & 61.2 & 7 & -4 & -40 & -1.0 & 0.3 & 15.6 & & 3 & 10 & -1 & -4 & -2 \\
\hline MMDB5 I 7R & -10 & 4.7 & 83 & 9.1 & 2 & -4 & 92.2 & 14 & -4 & -40 & -1.0 & 0.4 & 14.8 & 57 & 3 & 20 & -1 & -4 & 2 \\
\hline MMDB5I8R & 34 & 3.2 & 141 & 4.2 & 3 & 11 & 130.5 & 7 & -4 & -40 & -1.0 & -0.2 & & 427 & 3 & 11 & -1 & -4 & -2 \\
\hline MMDB5ISR & 167 & 3.0 & 103 & 10.4 & 4 & 7 & 128.2 & -4 & 5 & -40 & 1.0 & 0.3 & 17.4 & 1106 & 6 & 17 & -1 & 69 & -2 \\
\hline MMDB520R & 287 & 5.1 & 220 & 39.6 & 48 & 24 & 308.7 & -4 & -4 & -40 & 1.0 & -0.2 & 16.3 & 2046 & 8 & -4 & -1 & 1116 & -2 \\
\hline MMDB52 IR & -10 & 4.7 & 237 & 1.4 & 4 & 49 & 189.6 & -4 & -4 & -40 & -1.0 & 0.4 & & & 3 & 7 & -1 & -4 & -5 \\
\hline MMDB522R & -10 & 5.4 & 132 & 18.2 & 3 & -4 & 144.1 & 10 & 4 & -40 & 1.0 & 0.3 & 13.5 & 1331 & 5 & 10 & -1 & 134 & -2 \\
\hline MMDC50 IR & 41 & 4.7 & 84 & 10.9 & 4 & -4 & 93.4 & -4 & -4 & -40 & -1.0 & -0.2 & 13.3 & & 5 & 15 & -1 & -4 & -2 \\
\hline MMDC502R & 40 & 4.7 & 79 & 13.1 & 3 & 4 & 91.0 & -4 & -4 & -40 & -1.0 & -0.2 & 14.7 & & 5 & 11 & -1 & -4 & -5 \\
\hline MMDC503R & 389 & 4.6 & 73 & 7.9 & 10 & -4 & 108.1 & -4 & -4 & -40 & -1.0 & -0.2 & 15.1 & 40 & 9 & 23 & -1 & -4 & -5 \\
\hline MMDC504R & 20 & 4.2 & 100 & 10.5 & 3 & -4 & 106.4 & 9 & -4 & -40 & -1.0 & $0 . \overline{3}$ & 15.3 & & 6 & 11 & -1 & -4 & -2 \\
\hline MMDC & 145 & 6.1 & 61 & 12.8 & 27 & -4 & 70.1 & -4 & 10 & -40 & -1.0 & -0.2 & 15.3 & 703 & 3 & 5 & -1 & -4 & -2 \\
\hline MMDC506R & -10 & 5.5 & 114 & 14.1 & 2 & -4 & 125.9 & -4 & -4 & -40 & 1.0 & 0.3 & 15.2 & 1173 & 5 & 9 & -1 & -4 & -2 \\
\hline MMOC507R & -10 & 7.4 & 221 & 43.0 & 14 & -4 & 212.2 & 6 & -4 & -40 & 1.0 & -0.2 & & 185 & 7 & 4 & -1 & 771 & -2 \\
\hline $\begin{array}{l}\text { MMDC508R } \\
\text { MMC509R }\end{array}$ & $\begin{array}{r}-10 \\
29\end{array}$ & $\begin{array}{l}4.9 \\
5.8\end{array}$ & $\begin{array}{l}130 \\
166\end{array}$ & $\begin{array}{l}21.5 \\
31.4\end{array}$ & $\begin{array}{l}3 \\
4\end{array}$ & $\begin{array}{l}-4 \\
-4\end{array}$ & $\begin{array}{l}114.9 \\
144.7\end{array}$ & $\begin{array}{l}-4 \\
-4\end{array}$ & $\begin{array}{r}-4 \\
4\end{array}$ & $\begin{array}{l}-40 \\
-40\end{array}$ & $\begin{array}{r}-1.0 \\
1.0\end{array}$ & $\begin{array}{l}-0.2 \\
-0.2\end{array}$ & $\begin{array}{l}15.9 \\
15.4\end{array}$ & $\begin{array}{r}864 \\
1331\end{array}$ & $\begin{array}{l}4 \\
7\end{array}$ & $\begin{array}{l}7 \\
5\end{array}$ & $\begin{array}{l}-1 \\
-1\end{array}$ & $\begin{array}{l}23 \\
-4\end{array}$ & $\begin{array}{l}-5 \\
-5\end{array}$ \\
\hline 'MUL SUSR & $c y$ & 3.0 & 100 & ד. דו. & 7 & ד & 67.6 & -4 & $T_{T}$ & 90 & 1.0 & U.L & ז & ל & & & & $\boldsymbol{T}$ & \\
\hline
\end{tabular}


AFPENDIX II TABULATION OF KEY FIELD MEASUREMENTS AND ANALYTICAL DATA---GROUND WATER---

AFPENDIX II TABULATION OF KEY FIELD MEASUREMENTS
ELEMTAL CONCENTRA-IONS IN PPB. ALK IN MEQ/L. COND IN UM/CM.

\begin{tabular}{|c|c|c|c|c|c|c|c|c|}
\hline RL I. D. & DOE I.D. & $\mathrm{PH}$ & EOND & ALK & $\mathrm{TH}$ & $A G$ & AL & AS \\
\hline 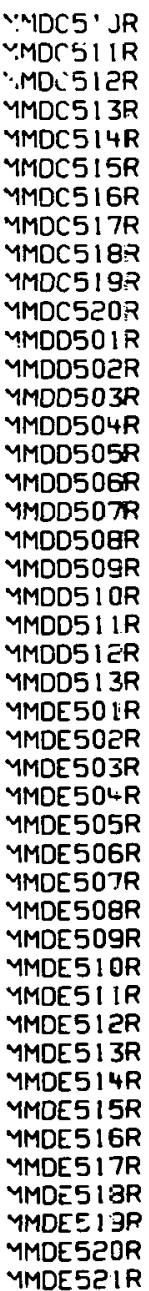 & 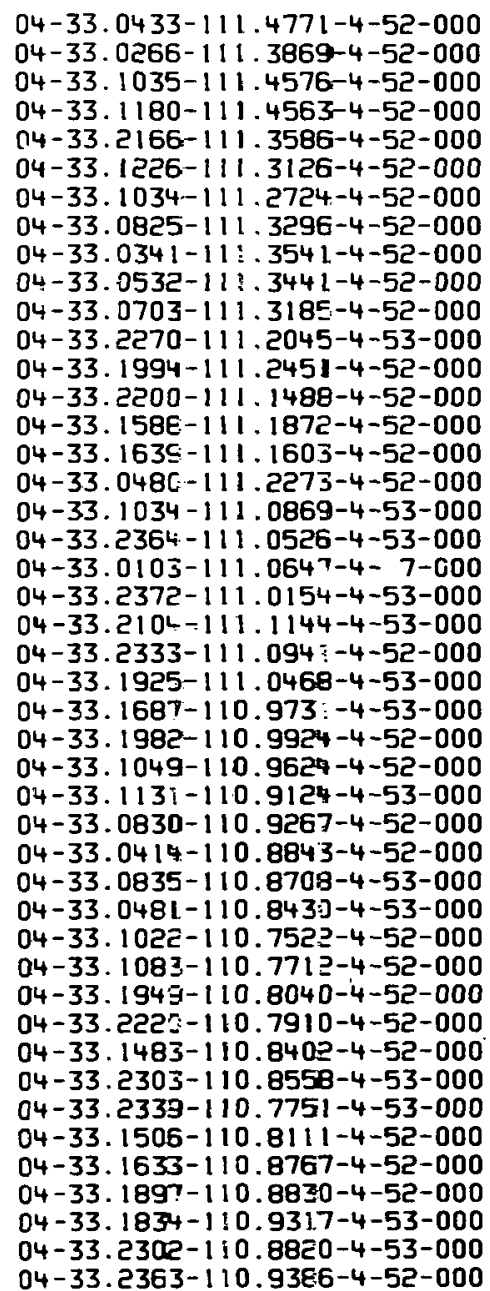 & 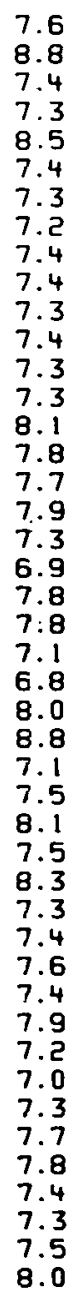 & $\begin{array}{r}2100 \\
800 \\
1700 \\
1500 \\
650 \\
900 \\
1400 \\
4100 \\
1350 \\
1625 \\
1525 \\
525 \\
1260 \\
650 \\
800 \\
500 \\
2600 \\
1250 \\
320 \\
600 \\
600 \\
230 \\
600 \\
500 \\
560 \\
600 \\
4490 \\
1140 \\
458 \\
2750 \\
910 \\
2250 \\
700 \\
570 \\
800 \\
610 \\
510 \\
428 \\
420 \\
413 \\
487 \\
461 \\
590 \\
690 \\
432\end{array}$ & $\begin{array}{l}2.10 \\
1.50 \\
1.40 \\
1.60 \\
1.80 \\
2.00 \\
2.20 \\
3.70 \\
2.70 \\
2.30 \\
2.30 \\
2.30 \\
4.80 \\
4.10 \\
2.30 \\
2.20 \\
4.10 \\
4.10 \\
2.70 \\
3.10 \\
3.90 \\
1.10 \\
3.00 \\
3.30 \\
4.20 \\
3.30 \\
4.90 \\
4.30 \\
3.80 \\
3.40 \\
3.20 \\
2.20 \\
3.00 \\
4.90 \\
E .90 \\
4.10 \\
3.20 \\
3.30 \\
2.20 \\
3.50 \\
5.30 \\
3.30 \\
5.40 \\
6.10 \\
3.80\end{array}$ & $\begin{array}{r}7 \\
8 \\
-5 \\
5 \\
-5 \\
9 \\
18 \\
13 \\
-5 \\
-5 \\
8 \\
6 \\
7 \\
14 \\
9 \\
-5 \\
-5 \\
7 \\
8 \\
-5 \\
23 \\
12 \\
-5 \\
9 \\
71 \\
7 \\
256 \\
142 \\
44 \\
226 \\
103 \\
281 \\
111 \\
70 \\
118 \\
99 \\
56 \\
48 \\
56 \\
49 \\
39 \\
67 \\
80 \\
74 \\
6\end{array}$ & $\begin{array}{r}-2 \\
-2 \\
-2 \\
-2 \\
-2 \\
2 \\
4 \\
2 \\
2 \\
-2 \\
-2 \\
-2 \\
-2 \\
3 \\
2 \\
-2 \\
-2 \\
2 \\
2 \\
-2 \\
5 \\
4 \\
-2 \\
2 \\
5 \\
-2 \\
6 \\
7 \\
2 \\
10 \\
2 \\
6 \\
5 \\
2 \\
7 \\
7 \\
3 \\
-2 \\
2 \\
-2 \\
-2 \\
5 \\
6 \\
5 \\
-2\end{array}$ & $\begin{array}{r}86 \\
46 \\
80 \\
53 \\
62 \\
11 \\
-10 \\
73 \\
41 \\
25 \\
10 \\
32 \\
58 \\
33 \\
13 \\
37 \\
58 \\
87 \\
117 \\
14 \\
67 \\
145 \\
81 \\
59 \\
12 \\
31 \\
79 \\
-10 \\
23 \\
35 \\
34 \\
29 \\
14 \\
-10 \\
41 \\
126 \\
27 \\
41 \\
39 \\
41 \\
61 \\
-10 \\
17 \\
40 \\
21\end{array}$ & $\begin{array}{r}1.2 \\
1.7 \\
1.3 \\
1.4 \\
2.5 \\
0.6 \\
2.6 \\
1.6 \\
1.3 \\
1.0 \\
1.7 \\
1.1 \\
1.0 \\
1.3 \\
-0.5 \\
2.6 \\
3.5 \\
2.8 \\
5.5 \\
1.4 \\
0.5 \\
3.9 \\
1.4 \\
0.6 \\
1.1 \\
6.0 \\
2.3 \\
1.8 \\
-0.5 \\
1.1 \\
3.9 \\
8.4 \\
2.4 \\
0.5 \\
1.0 \\
-0.5 \\
-0.5 \\
0.5 \\
1.8 \\
-0.5 \\
0.7 \\
-0.5 \\
-0.5 \\
-0.5 \\
-0.5\end{array}$ \\
\hline
\end{tabular}

B BA BE

$\begin{array}{rrr}195 & 92 & -1 \\ 107 & 10 & -1 \\ 125 & 60 & -1 \\ 121 & 30 & -1 \\ 104 & 28 & -1 \\ 127 & 6 & -1 \\ 202 & 29 & -1 \\ 349 & 77 & -1 \\ 285 & 44 & -1 \\ 202 & 61 & -1 \\ 223 & 28 & -1 \\ 81 & 205 & -1 \\ 144 & 137 & -1 \\ 70 & 78 & -1 \\ 139 & 43 & -1 \\ 80 & 148 & -1 \\ 401 & 51 & -1 \\ 166 & 154 & -1 \\ 39 & 51 & -1 \\ 90 & 29 & -1 \\ 40 & 30 & -1 \\ 34 & 4 & -1 \\ 49 & 37 & -1 \\ 79 & 93 & -1 \\ 39 & 37 & -1 \\ 172 & 6 & -1 \\ 421 & 52 & 1 \\ 102 & 87 & -1 \\ 78 & 163 & -1 \\ 295 & 138 & -1 \\ 103 & 42 & -1 \\ 79 & 35 & -1 \\ 72 & 41 & -1 \\ 52 & 40 & -1 \\ 92 & 29 & 1 \\ 66 & 66 & -1 \\ 41 & 12 & -1 \\ 42 & 130 & -1 \\ 28 & 72 & -1 \\ 28 & 23 & -1 \\ 33 & 48 & -1 \\ 34 & 12 & -1 \\ 42 & 37 & -1 \\ 90 & 43 & -1 \\ 34 & 23 & -1 \\ & & \end{array}$
CA CE CO CR

$\begin{array}{rrrrr}214 & -30 & -2 & 4 & 6 \\ 70 & -30 & -2 & -4 & 6 \\ 161 & -30 & -2 & -4 & 5 \\ 122 & -30 & 3 & 4 & 5 \\ 60 & -30 & -2 & 4 & 61 \\ 78 & -30 & 2 & 4 & 13 \\ 106 & -30 & 2 & 5 & 8 \\ 416 & -30 & -2 & 4 & 34 \\ 147 & -30 & -2 & -4 & 9 \\ 186 & -30 & -2 & -4 & 13 \\ 132 & -30 & -2 & -4 & 2 \\ 76 & -30 & -2 & -4 & 10 \\ 120 & -30 & -2 & -4 & 16 \\ 91 & -30 & -2 & 4 & 9 \\ 62 & -30 & 2 & -4 & 14 \\ 64 & -30 & -2 & -4 & 15 \\ 176 & -30 & -2 & -4 & 16 \\ 132 & -30 & -2 & -4 & 11 \\ 85 & -30 & 2 & -4 & 8 \\ 93 & -30 & -2 & -4 & 2 \\ 103 & 43 & 3 & 8 & 6 \\ 18 & -30 & -2 & 5 & 11 \\ 72 & -30 & -2 & -4 & 8 \\ 72 & -30 & -2 & -4 & 24 \\ 67 & 42 & 4 & 7 & 9 \\ 10 & -30 & -2 & -4 & 9 \\ 370 & 110 & 13 & 11 & 19 \\ 162 & 87 & 8 & 11 & 7 \\ 47 & -30 & 3 & 5 & 5 \\ 258 & 128 & 14 & 14 & 13 \\ 116 & 42 & 5 & 5 & 10 \\ 457 & 146 & 9 & 11 & 12 \\ 117 & 65 & 7 & 11 & 8 \\ 79 & -30 & 4 & 5 & 44 \\ 118 & 67 & 11 & 10 & 39 \\ 108 & 60 & 12 & 9 & 48 \\ 59 & 34 & 5 & 7 & 21 \\ 62 & -30 & -2 & -1 & 47 \\ 57 & -30 & 4 & 4 & 17 \\ 62 & -30 & 3 & -4 & 4 \\ 63 & -30 & 2 & -4 & 10 \\ 71 & 40 & 8 & 5 & 3 \\ 81 & 43 & 5 & 6 & 6 \\ 85 & -30 & 8 & 6 & 4 \\ 61 & -30 & -2 & -4 & 19\end{array}$


APPENDIX II TABULATION OF KEY FIELD MEASUREMENTS AND ANALYTICAL DATA--- GROUND WATER--=E LI MN MO NB NI P SC SE SR TI V Y ZN AND ZR CONCENTRATIONS. IN PPB.
CA K MG NA AND $I I$ CONCENTRATIONS IN PPM.

CA K MG NA AND SI CONCENTRATIONS IN PPM.

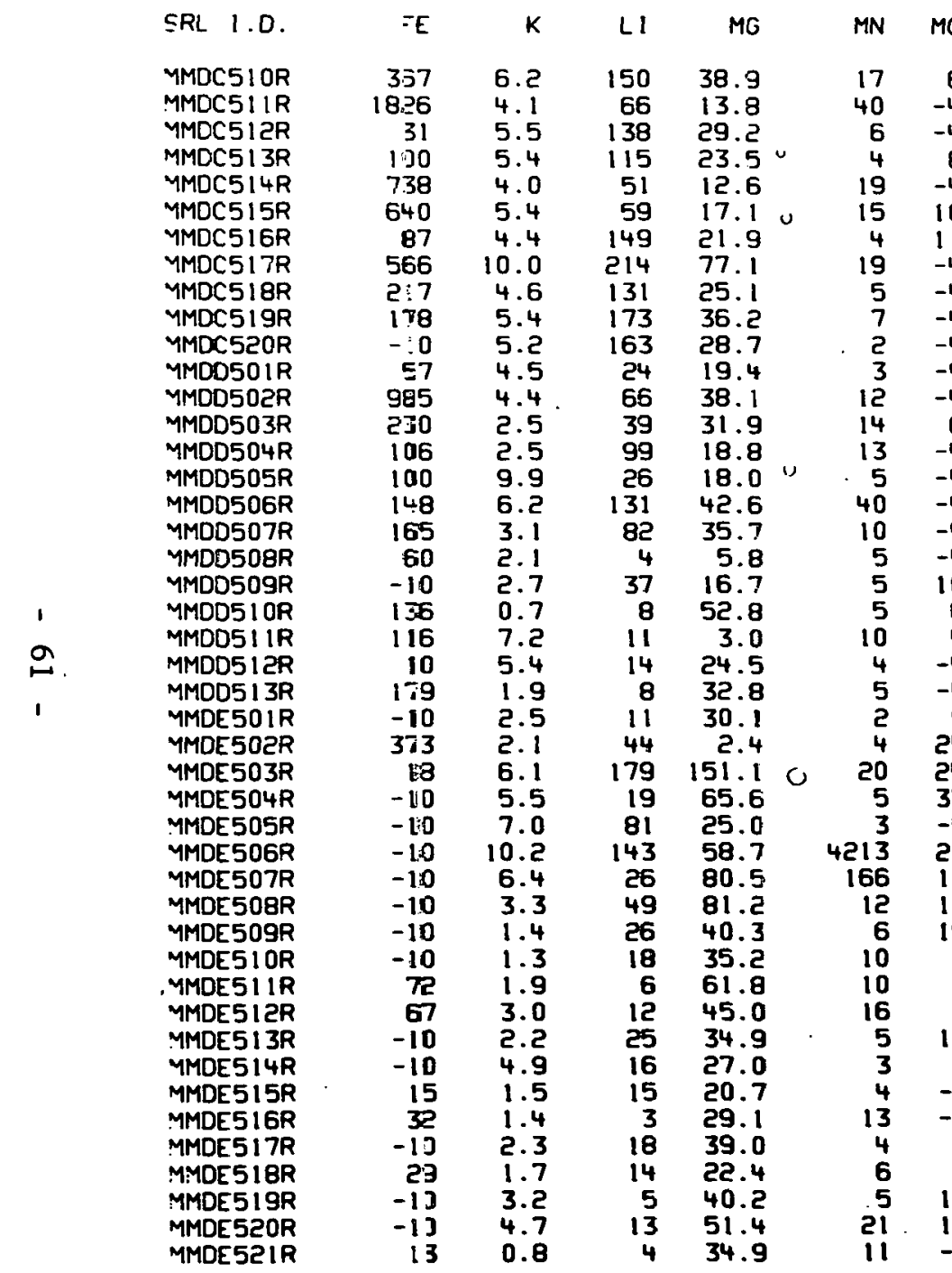

$\begin{array}{rrrrr}M N & M O & N A & N B & N 1 \\ 17 & 6 & 146.6 & -4 & 4 \\ 40 & -4 & 65.8 & 5 & 7 \\ 6 & -4 & 135.5 & -4 & 7 \\ 4 & 8 & 116.5 & -4 & 6 \\ 19 & -4 & 64.5 & -4 & -4 \\ 15 & 10 & 64.8 & 4 & 4 \\ 4 & 11 & 121.2 & -4 & 8 \\ 19 & -4 & 262.9 & -4 & -4 \\ 5 & -4 & 121.8 & -4 & -4 \\ 7 & -4 & 140.8 & -4 & -4 \\ 2 & -4 & 159.2 & -4 & 4 \\ 3 & -4 & 31.9 & -4 & -4 \\ 12 & -4 & 78.9 & -4 & 4 \\ 14 & 6 & 48.6 & -4 & 8 \\ 13 & -4 & 92.1 & 7 & -4 \\ 5 & -4 & 31.1 & -4 & -4 \\ 40 & -4 & 282.1 & -4 & -4 \\ 10 & -4 & 124.8 & -4 & -4 \\ 5 & -4 & 4.2 & 5 & -4 \\ 5 & 10 & 37.7 & 8 & -4 \\ 5 & 6 & 7.7 & 17 & 6 \\ 10 & 4 & 27.9 & 5 & 8 \\ 4 & -4 & 25.2 & -4 & 4 \\ 5 & -4 & 13.8 & -4 & 8 \\ 2 & 9 & 18.1 & 17 & 13 \\ 4 & 25 & 121.7 & -4 & -4 \\ 20 & 24 & 404.3 & 51 & 16 \\ 5 & 37 & 80.4 & 30 & 10 \\ 3 & -4 & 45.9 & 5 & 11 \\ 4213 & 21 & 296.6 & 46 & 14 \\ 166 & 12 & 47.6 & 22 & 10 \\ 12 & 18 & 38.2 & 44 & 14 \\ 6 & 15 & 30.6 & 21 & 11 \\ 10 & 5 & 28.5 & 19 & 4 \\ 10 & 6 & 36.4 & 24 & 8 \\ 16 & 8 & 39.9 & 23 & 25 \\ 5 & 17 & 21.3 & 20 & 9 \\ 3 & 5 & 20.6 & 13 & -4 \\ 4 & -4 & 37.1 & -4 & -4 \\ 13 & -4 & 24.2 & 4 & 4 \\ 4 & 4 & 20.0 & -4 & 4 \\ 6 & 5 & 19.7 & 10 & -4 \\ 55 & 13 & 10.8 & 25 & 10 \\ 21 & 16 & 25.2 & 20 & 13 \\ 11 & -4 & 23.6 & 4 & 4\end{array}$

\begin{abstract}
P SC
\end{abstract}
$\begin{array}{rr}-40 & 1.0 \\ -40 & 1.0\end{array}$

$\begin{array}{lll}0.5 & 15.4 & 1690\end{array}$

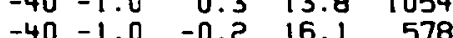

$\begin{array}{lllll}-40 & 1.0 & -0.2 & 13.2 & 630\end{array}$

$\begin{array}{lllll}-40 & 1.0 & 0.4 & 17.0 & 733 \\ -40 & 2 & 0.4 & 17.4 & 4486\end{array}$

$\begin{array}{lllll}-40 & 2.0 & 0.4 & 17.4 & 4486 \\ -40 & 1.0 & 0.3 & 14.7 & 1151\end{array}$

$\begin{array}{lllll}-40 & 1.0 & 0.4 & 15.6 & 1265\end{array}$

$\begin{array}{lllll}-40 & 1.0 & 0.3 & 15.6 & 975\end{array}$

$\begin{array}{lllll}42 & -1.0 & 0.3 & 9.8 & 372\end{array}$

$\begin{array}{lllll}-40 & -1.0 & 0.2 & 17.2 & 905\end{array}$

$\begin{array}{lllll}51 & 1.0 & 0.3 & 13.7 & 327\end{array}$

$\begin{array}{rrrrr}-40 & 1.0 & 0.6 & 12.8 & 1337 \\ 41 & -1.0 & 0.2 & 13.6 & 496\end{array}$

$\begin{array}{rrrrr}41 & -1.0 & 0.2 & 13.6 & 496 \\ -40 & 1.0 & 0.5 & 20.0 & 2025\end{array}$

$\begin{array}{lllll}101 & 1.0 & 0.5 & 24.6 & 2090\end{array}$

$\begin{array}{lllll}-40 & -1.0 & 0.5 & 4.9 & 184\end{array}$

$\begin{array}{lllll}-40 & 1.0 & 0.2 & 16.4 & 576\end{array}$

$\begin{array}{lllll}-40 & 1.0 & 0.3 & 7.0 & 210\end{array}$

$\begin{array}{lllll}-40 & -1.0 & 0.3 & 27.2 & 324\end{array}$

$\begin{array}{lllll}68 & 1.0 & 0.3 & 7.8 & 256\end{array}$

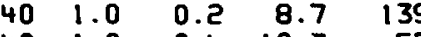

$\begin{array}{rrrrr}-40 & -1.0 & 0.4 & 10.3 & 57 \\ 92 & 1.0 & 0.4 & 26.9 & 2010\end{array}$

$\begin{array}{lllll}-40 . & 1.0 & 0.4 & 15.0 & 870\end{array}$

$\begin{array}{lllll}-40 & -1.0 & 0.4 & 17.9 & 529\end{array}$

$\begin{array}{lllll}65 & 1.0 & 0.2 & 12.2 & 2283\end{array}$

$\begin{array}{lllll}-40 & 1.0 & 0.3 & 19.0 & 2503\end{array}$

$\begin{array}{lllll}-40 & 1.0 & 0.4 & 18.1 & 613\end{array}$

$\begin{array}{lllll}42 & -1.0 & -0.2 & 16.4 & 381\end{array}$

$\begin{array}{lllll}56 & 1.0 & 0.2 & 16.0 & 464\end{array}$

$44 \quad 1.0 \quad 0.3 \quad 14.9$

$-40-1.0$

$51-1.0$

0.314 .9

$-40-1.0 \quad 0.2 \quad 16.1$

$\begin{array}{llll}-40 & 1.0 & 0.3 & 14.4\end{array}$

$-401.0$

$52-1.0$
$-40-1.0$ $\begin{array}{lllll}-40 & 1.0 & 0.4 & 17.8 & 435\end{array}$

$\begin{array}{lllll}48 & 1.0 & 0.2 & 16.3 & 428\end{array}$
MESSA SHEE T

$\begin{array}{rrrrr}T I & V & Y & Z N & Z R \\ 9 & 7 & -1 & -4 & -2 \\ 9 & 4 & -1 & 8 & -2 \\ 4 & 4 & -1 & -4 & -2 \\ 6 & 15 & -1 & -4 & -2 \\ 4 & -2 \\ 4 & 18 & -1 & -4 & -2 \\ 4 & 10 & 1 & 151 & 2 \\ 4 & 17 & 1 & 24 & 4 \\ 14 & 12 & 1 & 1489 & -2 \\ 7 & -4 & -1 & -4 & -2 \\ 5 & -4 & -1 & -4 & -2 \\ 4 & 4 & -1 & 452 & -2 \\ 3 & -4 & -1 & -4 & -2 \\ 4 & 10 & -1 & 766 & -2 \\ 5 & 6 & 1 & 176 & 4 \\ 3 & -4 & 1 & 67 & -2 \\ 3 & -4 & -1 & 34 & -2 \\ 5 & 18 & -1 & 88 & -2 \\ 9 & 15 & 1 & -4 & -2 \\ 4 & -4 & 1 & 167 & -2 \\ 2 & 4 & -1 & 1337 & -2 \\ 4 & 12 & 2 & 15 & 5 \\ 5 & 11 & 1 & 36 & 4 \\ 4 & 12 & -1 & 163 & -2 \\ 5 & -4 & 2 & 298 & -2 \\ 4 & 20 & 2 & -4 & 3 \\ 2 & -4 & -1 & -4 & -2 \\ 10 & 58 & 5 & 175 & 7 \\ 6 & 25 & 3 & 35 & 5 \\ 2 & 9 & 1 & 51 & -2 \\ 8 & 34 & 4 & 41 & 10 \\ 3 & 32 & 2 & 19 & 3 \\ 8 & 56 & 5 & 33 & 6 \\ 4 & 33 & 3 & -4 & 6 \\ 3 & 20 & 1 & 1688 & -2 \\ 5 & 26 & 3 & 155 & 5 \\ 6 & 17 & 2 & 35 & 8 \\ 4 & 19 & 2 & 1053 & 3 \\ -2 & 7 & 1 & 63 & -2 \\ 2 & 9 & 1 & 26 & -2 \\ -2 & 10 & -1 & 139 & -2 \\ -2 & 16 & -1 & 157 & -2 \\ 4 & 12 & 1 & 76 & 4 \\ 3 & 18 & 2 & 35 & 5 \\ 3 & 24 & 2 & -4 & -3 \\ 2 & 5 & -1 & 34 & -2\end{array}$


EXTERNAL DISTRIBUTION

\section{Immediate:}

Copy

No.

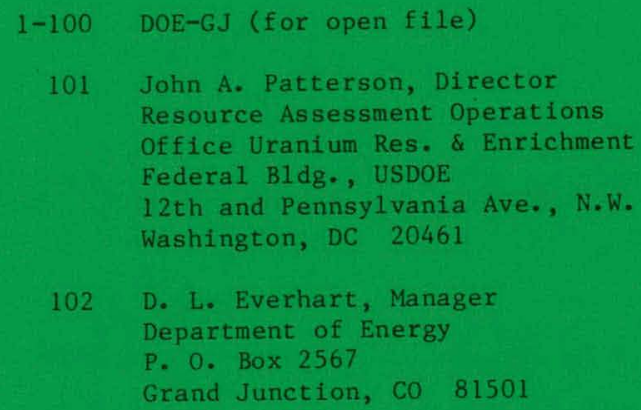

102 D. L. Everhart, Manager

Department of Energy

P. 0. Box 2567

Grand Junction, CO 81501

\section{Delayed (after DOE-GJO release)}

\section{Copy}

No.

\section{6-107 Terry W. Offield}

Branch of Uranium \& Thorium Resources USGS

MS 964

P. O. Box 25046

Denver Federal Center

Denver, CO 80225

108 John W. Arendt

Union Carbide Corp.

Nuclear Division - MS 246

P. 0. Box P

Oak Ridge, TN 37830

109-110 Manager

Technical Information Center

Department of Energy

P. O. Box E.

Oak Ridge, TN 37830
103 David H. Dahlem, Project Officer

NURE Project office

Department of Energy

P. 0. Box 2567

Grand Junction, CO 81501

104-105 Donald E. Livingston

Manager, Geochemical Department, DADD Bendix Field Engineering Corp.

P. 0. Box 1569

Grand Junction, CO 81501
111-112 W. Morris Group Leader, J-5

Los Alamos Scientific Laboratory MS 586

P. 0. Box 1663

Los Alamos, NM 87545

113 Dr. Ken Holt $j i$, Ass't Director USDA - Forest Service Watershed Unit 633 W. Wisconsin Ave. Milwaukee, WI 55202

114-119 DOE-SR

120-200 TIS File, SRL 